\title{
Ascent Aerodynamic Force and Moment Database Development for the Space Launch System
}

\author{
Patrick R. Shea* and Jeremy T. Pinier ${ }^{\dagger}$ \\ NASA Langley Research Center, Hampton, VA 23681, USA \\ Heather P. Houlden ${ }^{\ddagger}$, Amber L. Favaregh ${ }^{\S}$, and Michael J. Hemsch ${ }^{\mathbb{I}}$ \\ ViGYAN, Inc., Hampton, VA 23666, USA \\ Derek J. Dallell and Stuart E. Rogers** \\ NASA Ames Research Center, Moffett Field, CA 94035, USA \\ Jamie G. Meeroff ${ }^{\dagger \dagger}$ and Henry C. Lee ${ }^{\ddagger}$ \\ Science \& Technology Corp., Moffett Field, CA 94035, USA
}

\begin{abstract}
The Space Launch System Aerodynamics Task Team is responsible for delivering aerodynamic force and moment databases from liftoff through ascent until the rocket leaves the Earth's atmosphere. The process for developing the ascent portion of this database is described in the current paper. The data used to develop the database were generated using a combination of wind tunnel testing and CFD simulations. The details of the wind tunnel testing performed at the NASA Ames Unitary Plan Wind Tunnel and CFD simulations performed using FUN3D at wind tunnel and flight conditions are discussed, and comparisons of these data sets are provided. The methods used for converting the source data into the final database response surfaces with corresponding uncertainty are also detailed.
\end{abstract}

\section{Notice to the Reader}

The Space Launch System, including its predicted performance and certain other features and characteristics, have been defined by the U.S. Government to be Sensitive But Unclassified (SBU). Information deemed to be SBU requires special protection and may not be disclosed to an international audience. To comply with SBU restrictions, details such as absolute values have been removed from some plots and figures in this paper. It is the opinion of the authors that despite these alterations, there is no loss of meaningful technical content. Analytical methodologies and capabilities are discussed; significant and interesting technical results are still present; and meaningful conclusions are presented.

\section{Nomenclature}

$\begin{array}{llll}\text { Variables } & & C x & \text { Coefficient of } x \\ C A & \text { Axial Force Coefficient } & C Y & \text { Side Force Coefficient } \\ C L L & \text { Rolling Moment Coefficient } & P H I V & \text { Vertical Axis Roll Angle } \\ C L M & \text { Pitching Moment Coefficient } & U & \text { Uncertainty } \\ C L N & \text { Yawing Moment Coefficient } & U_{b a l} & \text { Balance Calibration Fit Uncertainty } \\ C N & \text { N } & U_{D B M n e t} & \text { Net Database Modeling Uncertainty }\end{array}$

CN Normal Force Coefficient

\footnotetext{
* Research Aerospace Engineer, Configuration Aerodynamics Branch, Mail Stop 499, AIAA Member.

${ }^{\dagger}$ Research Aerospace Engineer, Configuration Aerodynamics Branch, Mail Stop 499, AIAA Associate Fellow.

$\doteqdot$ Senior Aerospace Engineer, 30 Research Drive, AIAA Senior Member.

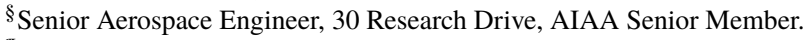

IConsultant, 30 Research Drive, AIAA Associate Fellow.

"Research Scientist/Eng., Computational Aerosciences Branch, AIAA Member.

**Aerospace Eng., Computational Aerosciences Branch, AIAA Associate Fellow.

${ }^{\dagger}$ Research Scientist/Eng., Computational Aerosciences Branch, AIAA Member.

${ }_{\ddagger}$ Research Scientist, Computational Aerosciences Branch, AIAA Member.
} 


\begin{tabular}{|c|c|c|c|}
\hline$U_{D B M}$ & Database Modeling Uncertainty & \multicolumn{2}{|c|}{ Abbreviations } \\
\hline$U_{G 2 F}$ & Ground to Flight Uncertainty & AMS & Angle Measurement System \\
\hline$U_{r p t}$ & Experimental Uncertainty & ATT & Aerodynamics Task Team \\
\hline$U_{\text {tstnet }}$ & Net Modeling Validation Uncertainty & CFD & Computational Fluid Dynamics \\
\hline$U_{t s t}$ & Modeling Validation Uncertainty & GOX & Gaseous Oxygen \\
\hline$U_{W T c o r r}$ & Wind Tunnel Corrections Uncertainty & LAS & Launch Abort System \\
\hline$U F$ & Uncertainty Factor & LMRM & Large Model Roll Mechanism \\
\hline$\alpha$ & Pitch Angle & LOX & Liquid Oxygen \\
\hline$\beta$ & Sideslip Angle & MPCV & Multi-Purpose Crew Vehicle \\
\hline$\Delta$ & Difference & OML & Outer Mold Line \\
\hline$\phi$ & Roll Angle & RANS & Reynolds Averaged Navier-Stokes \\
\hline Subscripts & & SLS & Space Launch System \\
\hline COR & Corrected & SRB & Solid Rocket Booster \\
\hline & Roll Sweep & TVC & Thrust Vector Control \\
\hline$U N C$ & Uncorrected & UPWT & Unitary Plan Wind Tunnel \\
\hline & & WT & Wind Tunnel \\
\hline
\end{tabular}

\section{Introduction}

THE Space Launch System (SLS) is a launch platform being developed by NASA to extend human exploration I beyond Earth's orbit [1]. The SLS vehicle, seen in Figure 1, is comprised of a liquid fuel core stage common to all of the vehicle configurations with two solid rocket boosters (SRBs) on the Block 1 configurations or evolved boosters on the Block 2 configurations. A number of upper stages can be integrated with the core stage depending on mission requirements including three configurations with the Orion Multi-Purpose Crew Vehicle (MPCV) for crewed missions. The SLS Aerodynamics Task Team (ATT) is tasked with providing a database of the aerodynamic forces and moments on each of the launch vehicles throughout the entire launch from liftoff until the vehicle leaves the Earth's atmosphere. One of the critical phases of flight for the vehicle as it leaves the atmosphere is the ascent portion of flight as the vehicle passes through Mach 1 and maximum dynamic pressure when aerodynamic loads are largest on the vehicle and the flow physics can change rapidly.

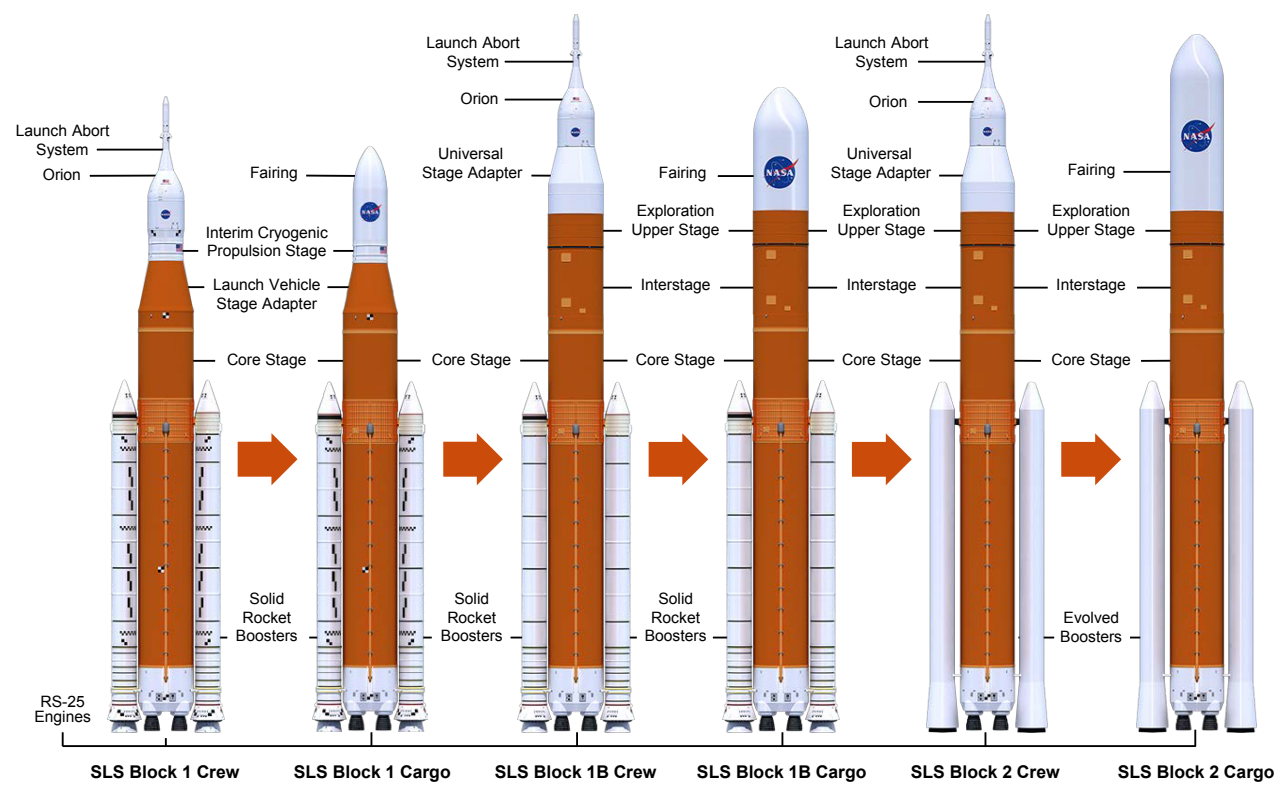

Fig. 1 Artist renderings of the SLS vehicle configurations [2].

The methods used for the current SLS Block 1B crew vehicle ascent force and moment database development are based on methods developed previously for the Ares 1 and SLS Block 1 crew vehicles [3-5]. These databases were 
comprised mainly of wind tunnel data for the nominal database values, and CFD solutions were used to help guide the uncertainty quantification. For the current effort, more CFD solutions were available than during the previous database development efforts, which allowed for more advanced wind tunnel to CFD comparisons to be made following the work of Hemsch [6].

The focus of the current paper will be the development of the ascent force and moment database for the SLS Block 1B crew vehicle configuration. This database was developed using wind tunnel data from the NASA Ames Unitary Plan Wind Tunnel, CFD simulations of the vehicle at wind tunnel conditions, and CFD simulations of the vehicle at flight conditions. The sections that follow will discuss the details of the wind tunnel test and CFD simulations, the development of the database response surfaces, the comparison of the WT and CFD simulations, and outline the methodology used for quantifying the uncertainty of the forces and moments delivered in the final database.

\section{Experimental Setup}

\section{A. NASA Ames Unitary Plan Wind Tunnel (UPWT)}

The NASA Ames UPWT (Fig. 2) is comprised of two interconnected wind tunnels that share a main drive [7-9]. The two wind tunnels are the 11-Foot Transonic Wind Tunnel and the 9- by 7-Foot Supersonic Wind Tunnel, and both were utilized in the current test effort.

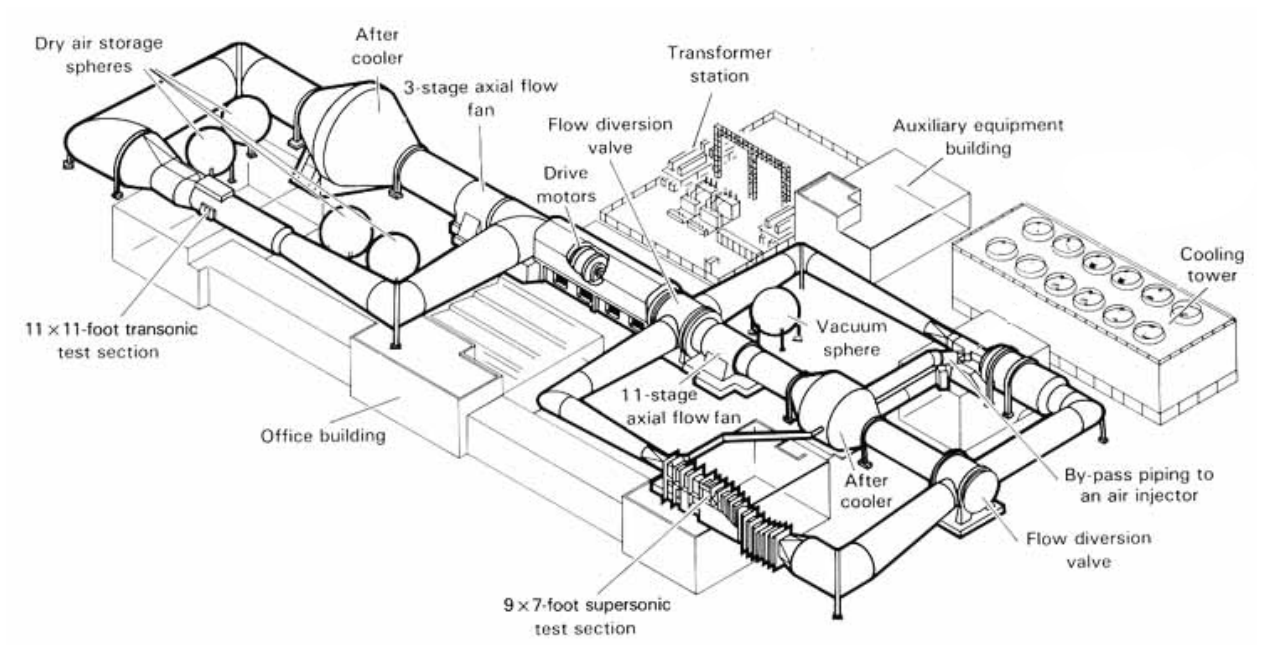

Fig. 2 NASA Ames UPWT site layout.

\section{11-Foot Transonic Wind Tunnel}

The 11-Foot Transonic Wind Tunnel (11-Foot TWT) is a closed circuit, variable pressure, continuous operation tunnel. The test section has a square cross-section of 11 feet, a length of 22 feet, and has $6 \%$ porosity slotted walls. For the current test, the SLS model was supported using the traversing strut located downstream of the test section. The strut is able to translate vertically and supports a knuckle and sleeve mechanism that can set model attitude within a $15^{\circ}$ half-angle cone (pitch and yaw). The 11-Foot TWT can operate at continuously variable Mach numbers ranging from 0.20 to 1.40 [7,8]. For the current test, the tunnel was operated between Mach 0.30 and 1.30 at unit Reynolds numbers of $5 \times 10^{6}$ per foot.

\section{9- by 7-Foot Supersonic Wind Tunnel}

The 9- by 7-Foot Supersonic Wind Tunnel (9- by 7-Foot SWT) is a closed circuit, variable pressure, continuous operation tunnel. The test section has a rectangular cross-section that is 7 feet high, 9 feet wide, and 18 feet long. The model support system is a traversing strut located downstream of the test section. The strut is able to translate horizontally and supports a knuckle and sleeve mechanism that can set model attitude within a $15^{\circ}$ half-angle cone (pitch and yaw). The 9- by 7-Foot SWT can operate at continuously variable Mach numbers ranging from 1.54 to 2.56 
by setting an asymmetric sliding nozzle block [7]. For the current test, the tunnel was operated between Mach 1.60 and 2.50 at unit Reynolds numbers of $3 \times 10^{6}$ and $5 \times 10^{6}$ per foot.

\section{B. Model Installation}

The SLS test model was sting mounted in both wind tunnels, and model positioning relied on the knuckle-sleeve resolvers, traversing struts, and the large model roll mechanism (LMRM) in the 11-Foot TWT. A $0^{\circ}$ primary adapter was used to connect the UPWT SR-228 sting to the LMRM or knuckle-sleeve mechanism in the 11-Foot TWT and 9by 7-Foot SWT, respectively. Due to the unique taper on the MC-12H-1.75A balance, a custom balance adapter was fabricated to interface between the balance and sting. Figure 3 shows the SLS Block 1B crew vehicle model installed in the test section of the 11-Foot TWT with the model painted for PSP testing. The details of the PSP are presented in the paper by McMillin et al. [10].

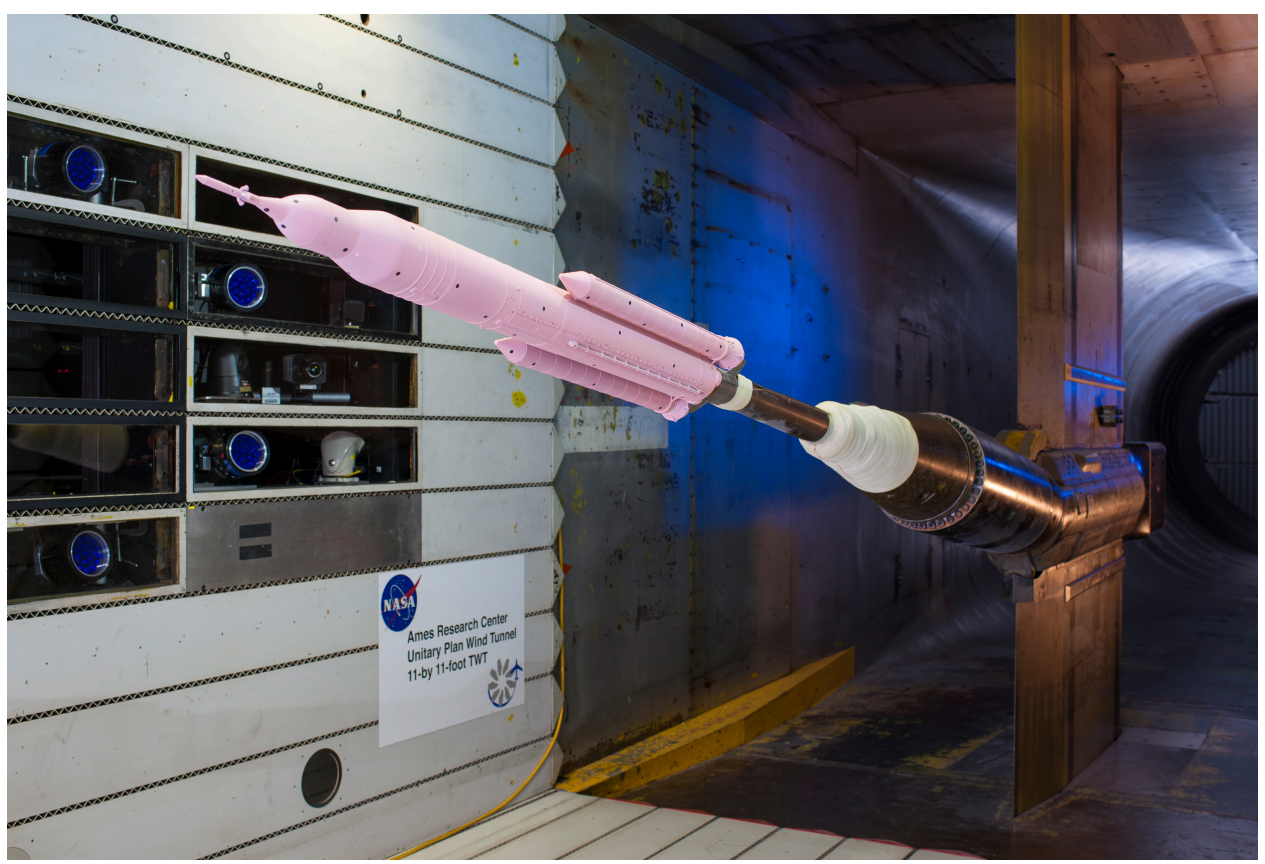

Fig. 3 SLS Block 1B crew vehicle test model installed in the NASA Ames UPWT 11-Foot Transonic Wind Tunnel. (Photo credit: NASA/Dominic Hart)

\section{Model Description}

The wind tunnel model was a 1.3\%-scale model of the full-scale SLS vehicle designed with a specific focus on acquiring force and moment data in the transonic flow regime. The model design was based on the most up-to-date flight outer mold line (OML) of the SLS Block 1B Crew (also referred to as the SLS-28k) vehicle with standard simplifications and modifications for scaled fabrication. The test hardware was supported in the wind tunnel test sections using a traditional sting-mount, and was designed in a modular fashion to accommodate testing multiple SLS geometries including a number of the geometries seen in Figure 1. The geometries shared a common core that remained attached to the sting/balance interface throughout the course of testing. Images taken from the CAD model of the common core with SRBs and assembled SLS-28k can be seen in Figures 4(a) and 4(b), respectively.

The model scale ( $1.3 \%$ of full scale) was selected with three main constraints: 1) make the model as large as possible to allow for more detailed protuberances, 2) keep the model small enough to ensure that the test section would not choke at subsonic conditions, and 3) keep the model short enough to ensure that shock reflections at supersonic speeds (Mach $\geq 1.10$ ) would not interfere with the force and moment measurements [11, 12]. All protuberances considered to be significant for aerodynamic loading were included as part of the model design, and a number of these protuberances were removable to allow for clean test configurations. A list of the model protuberances can be found in Table 1. 


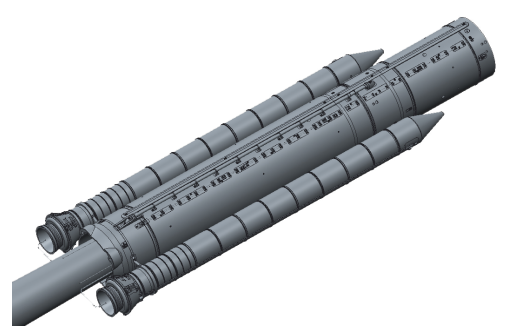

(a) Common Stage with SRBs

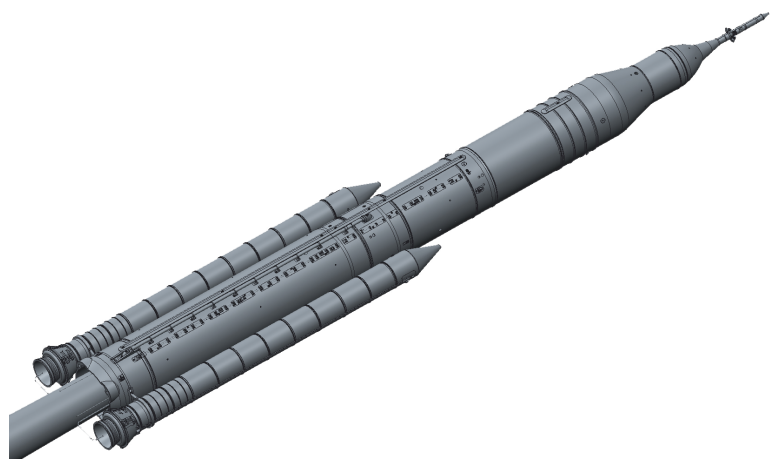

(b) SLS-28k

Fig. 4 CAD model of the $1.3 \%$ wind tunnel model.

Table 1 Model Protuberances.

\begin{tabular}{cc}
\hline Vehicle Element & Protuberances \\
\hline Systems Tunnel (1)* \\
LOX Feedline and Fairing (2)* \\
LOX Aft Attach Structure (2)* \\
Repressurization Lines (1)* \\
GOX Vent Cover (1)* \\
Cameras (6)* \\
Forward Stabilizers (2)* \\
Umbilical Attachment Points (4) \\
Systems Tunnel (1) \\
Rooster Tail (1) \\
Hold Down Posts (4)* \\
TVC Brackets (2)* \\
Kick Ring (1) \\
Aft Booster Attach Ring (1) \\
SRB Attach Hardware (Fwd/Aft) \\
Booster Separation Motors (8)* \\
LAS Tower (1) \\
LAS Nozzles (4)* \\
LAS Systems Tunnel (1)* \\
MPCV Umbilical (1)* \\
Upper Stage Systems Tunnel (1)* \\
Cameras (3)* \\
\hline SLS-28k Upper Stage
\end{tabular}

* Removable protuberance for clean configuration testing 


\section{Boundary Layer Grit Patterns}

The boundary layer on the wind tunnel model was tripped to force turbulent boundary layer development. Trip devices for SLS ascent testing are traditionally placed as rings at key axial stations, and the locations of these rings can be seen in Figure 5. Table 2 provides the details of the trip dot heights for each of the two test sections. Trip dot sizes were selected following the guidelines laid out by Braslow and Knox [13].

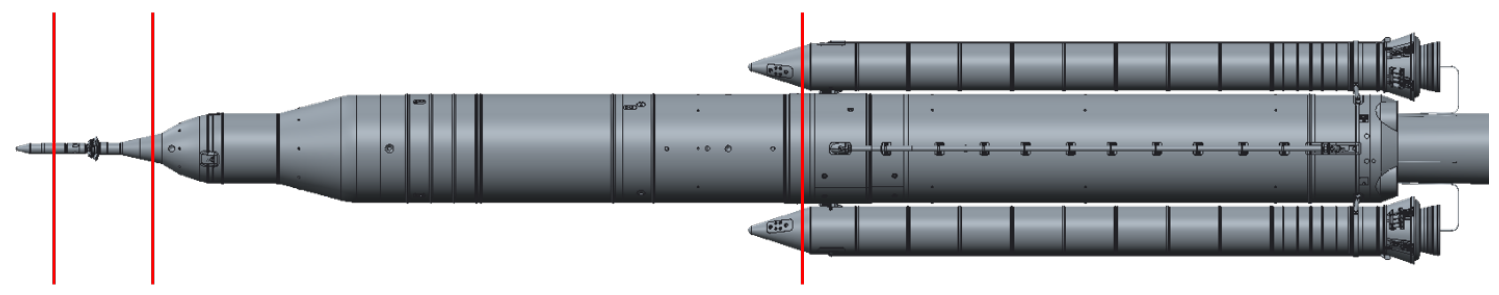

Fig. 5 Axial locations of the boundary layer trip dots for the SLS-28k geometry.

Table 2 Trip dot sizes and locations for the 11-Foot TWT and 9- by 7-Foot SWT.

\begin{tabular}{ccc}
\hline Location & Test Section & Trip Dot Height [in] \\
\hline LAS Tower & 11-Foot & 0.0056 \\
LAS/Orion Fairing & 11-Foot & 0.0080 \\
SRB Nose Cones & 11-Foot & 0.0060 \\
\hline LAS Tower & 9- by 7-Foot & 0.0075 \\
LAS/Orion Fairing & 9- by 7-Foot & 0.0114 \\
SRB Nose Cones & 9- by 7-Foot & 0.0086 \\
\hline
\end{tabular}

\section{Instrumentation}

\section{Force and Moment Measurements}

The balance used for the test was the Ames UPWT MC-12H-1.75A which is an internal, six component, sting mounted balance with a nominal diameter of 1.75 inches. Previous calibrations of the MC-12H-1.75A were either performed over custom load ranges, or were performed in situ for a specific model build up and were therefore not appropriate for the current test. With this in mind, a new calibration was performed by Triumph to calibrate the balance over the expected load range. The Ames UPWT test team developed the balance calibration matrix and assessed the calibration results using the BALFIT software package to develop the balance matrix [14, 15]. BALFIT was also used to independently verify the check loading performed in the model preparation area at the UPWT to ensure the balance was behaving as predicted and that the balance calibration matrix was implemented correctly.

\section{Base and Cavity Pressure Measurements}

Base pressure measurements were acquired for the core (4 ports) and for each of the SRBs (2 ports each). These pressure taps were attached external to the sting as opposed to the model as seen in Figure 6. The pressure ports were located approximately 0.25 inches inside the respective cavities. The 15 psi Measurement Specialties 16-MS Micro Scanner used for the base pressure measurements was housed in a protective case mounted on the sting upstream of the primary adapter. The 16-MS Micro Scanner has a quoted static accuracy of $\pm 0.05 \%$ of full scale. All of the necessary plumbing and wiring was routed so as not to bridge the balance, and the base pressure measurements were used to correct the force and moment measurements as described in Section V.A.1. 


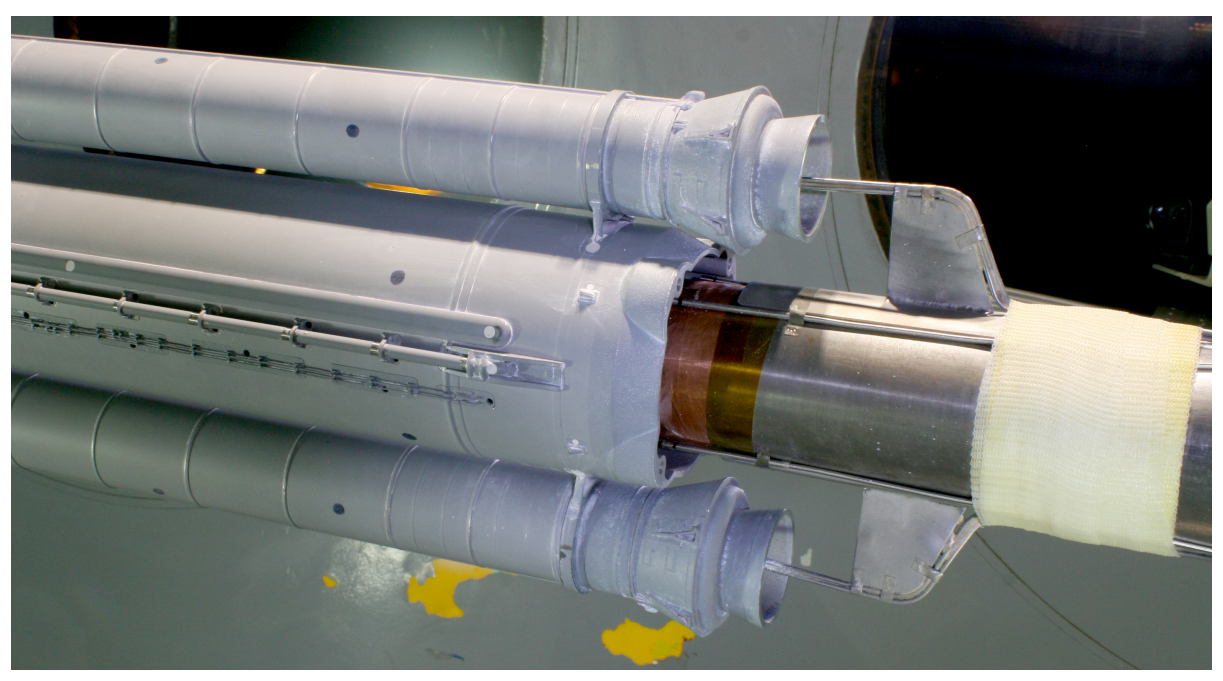

Fig. 6 Photo of the base pressure installation.

\section{Model Orientation}

The model orientation during the test was calculated using the angle resolvers in the knuckle-sleeve adapters and the large model roll mechanism (11-Foot TWT only). Sting deflection measurements were taken as part of the model build up and check loading process at the UPWT and were applied throughout the course of testing. Prior to testing, the model position measurements and calculations were verified in the test section using a FARO arm measurement system and an Angle Measurement System (AMS) package installed on a custom built model leveling plate. The FARO arm was used to assess the model height and angle measurements (pitch and yaw) relative to the tunnel centerline, and the AMS package was used to assess the model pitch and roll angles. The model positioning system was required to set the model orientation to within $\pm 0.1^{\circ}$ in pitch, yaw, and roll, and this tolerance was evaluated using the using the AMS and FARO arm measurements at wind-off conditions. The AMS and FARO arm measurements also served as verification of the model positioning calculations (sting bend angles, sting deflections, balance deflections, etc.) which are integral to the model positioning system. Since the LMRM was not able to be used in the 9- by 7-Foot SWT, the AMS package was used to set the model roll angle while setting the taper during model installation.

\section{Computational Simulations}

\section{A. Flow Solution Methodology}

Simulations were run using the CFD solver FUN3D at both the Ames UPWT test conditions and full-scale flight conditions. FUN3D was run on the wind tunnel geometry at conditions tested during the Ames UPWT test, and a set of FUN3D simulations were run on the full-scale flight geometry with the engine plumes simulated. For the wind tunnel condition simulations, solutions were developed running 2000 RANS iterations with the Spalart-Allmaras turbulence model. Grid adaptations were performed after iterations 2000 and 2500, followed by 0 to 5000 unsteady RANS iterations as necessary based on the iterative history of each case. The initial mesh was 32 million nodes, growing to 56 million nodes at completion. The same basic process was followed for the FUN3D simulations performed on the flight geometry at flight conditions, except the initial mesh consisted of 52 million nodes. Plumes for the four core engines and two boosters were modeled using air and a modified nozzle geometry to set the thrust and mass flow. Mach dependent conditions were set using reference trajectories from the SLS Guidance, Navigation, and Control team. Figure 7 shows two example solutions from the wind tunnel (Fig. 7(a)) and flight cases (Fig. 7(b)). Details of the FUN3D simulations can be found in the paper by Meeroff et al. [16].

\section{B. Force and Moment Data Extraction}

Force and moment data were extracted from the CFD solutions to compare with the wind tunnel measurements and for the development of the uncertainty quantification. The forward portion of the vehicle was always included in 


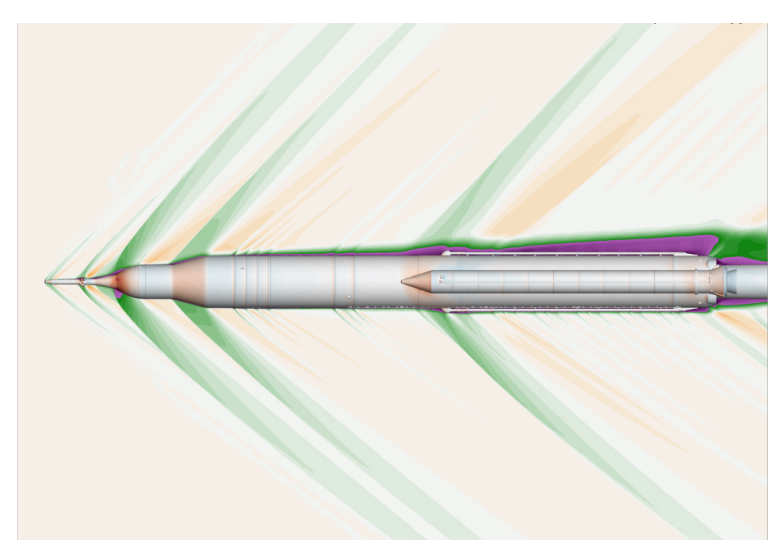

(a) Wind Tunnel

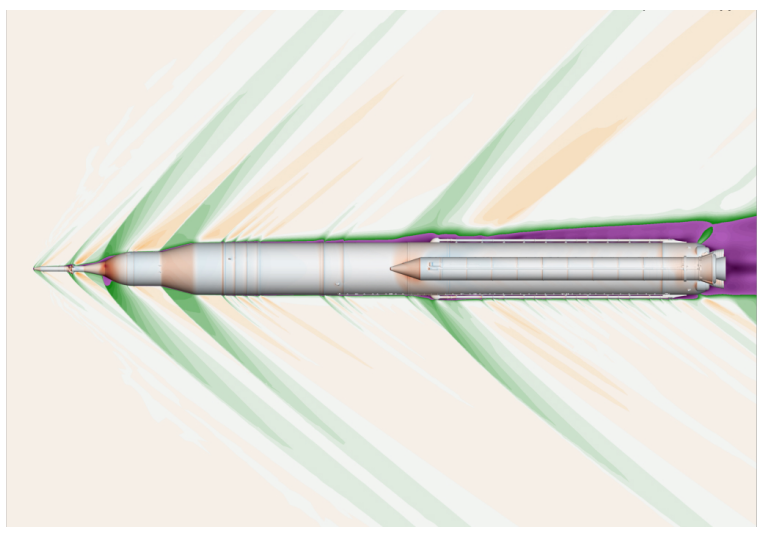

(b) Flight

Fig. 7 Example Mach and surface pressure contours for Block 1B crew vehicle wind tunnel and flight FUN3D simulations.

the surface integrations, but the base region of the vehicle was handled using different surface regions depending on the coefficient being computed. In Figure 8, the green surface extends to the nose of the vehicle (not shown) and is the forward portion of the vehicle that was included in all integrations. The blue region contains the SRB nozzles, which was included in all coefficients except axial force. This distinction was made in consideration of the SLS program definition of the force and moments. The orange and yellow regions are the base and cavity regions, respectively, that had analogous surfaces on the metric portion of the wind tunnel model. These two regions were always excluded from the CFD integrations, and were corrected for in the experimental measurements using the base pressure measurements. The brown region is the sting definition which was always excluded in the CFD integrations and was nonmetric in the wind tunnel tests. A summary of the integration surfaces used for the force and moment coefficients can be found in Table 3.

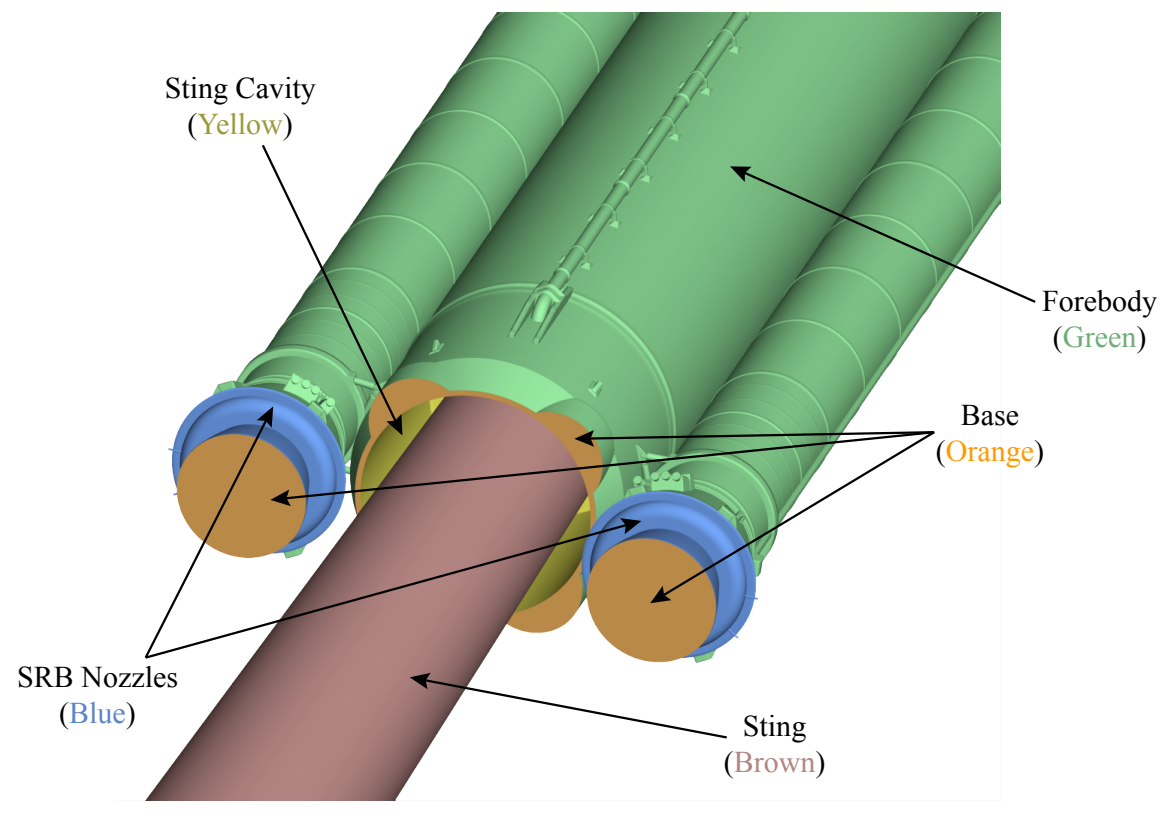

Fig. 8 Definition of FUN3D base region integration surfaces used for force and moment calculations. 
Table 3 FUN3D base region integration surfaces used for force and moment calculations. Colors are in reference to Figure 8.

\begin{tabular}{|c|c|c|c|c|c|}
\hline Coefficients & $\begin{array}{c}\text { Forebody } \\
(\text { Green })\end{array}$ & $\begin{array}{c}\text { SRB Nozzles } \\
(\text { Blue })\end{array}$ & $\begin{array}{c}\text { Base } \\
(\text { Orange })\end{array}$ & $\begin{array}{c}\text { Sting Cavity } \\
\text { (Yellow) }\end{array}$ & $\begin{array}{c}\text { Sting } \\
(\text { Brown })\end{array}$ \\
\hline $\begin{array}{c}\text { Normal Force } \\
(C N)\end{array}$ & $\mathrm{Y}$ & $\mathrm{Y}$ & $\mathrm{N}$ & $\mathrm{N}$ & $\mathrm{N}$ \\
\hline $\begin{array}{c}\text { Side Force } \\
(C Y)\end{array}$ & $\mathrm{Y}$ & $\mathrm{Y}$ & $\mathrm{N}$ & $\mathrm{N}$ & $\mathrm{N}$ \\
\hline $\begin{array}{c}\text { Axial Force } \\
(C A)\end{array}$ & $\mathrm{Y}$ & $\mathrm{N}$ & $\mathrm{N}$ & $\mathrm{N}$ & $\mathrm{N}$ \\
\hline $\begin{array}{c}\text { Rolling Moment } \\
(C L L)\end{array}$ & $\mathrm{Y}$ & $\mathrm{Y}$ & $\mathrm{N}$ & $\mathrm{N}$ & $\mathrm{N}$ \\
\hline $\begin{array}{c}\text { Pitching Moment } \\
(C L M)\end{array}$ & $\mathrm{Y}$ & $\mathrm{Y}$ & $\mathrm{N}$ & $\mathrm{N}$ & $\mathrm{N}$ \\
\hline $\begin{array}{c}\text { Yawing Moment } \\
(C L N)\end{array}$ & $\mathrm{Y}$ & $\mathrm{Y}$ & $\mathrm{N}$ & $\mathrm{N}$ & $\mathrm{N}$ \\
\hline
\end{tabular}

\section{Database Development}

\section{A. Wind Tunnel Data Corrections}

\section{Standard Corrections}

Standard corrections for force and moment testing were applied during testing in the wind tunnel facilities. These corrections included buoyancy, flow angularity, and base force corrections. Buoyancy corrections were applied using the Ames UPWT best practices based on the projected areas of the model in the test section and the facility static pressure calibrations. Flow angularity corrections were made in the vertical (pitch) plane of the 11-Foot TWT data, and vertical (yaw) and side flow (pitch) corrections were applied to the 9- by 7-Foot SWT data. These corrections were based on standard comparisons between upright $\left(\phi=0^{\circ}\right)$ and inverted $\left(\phi=180^{\circ}\right)$ runs, and corrections were applied to pitch angle, sideslip angle, pitching moment, and yawing moment. Additionally, base force corrections were applied in an effort to remove the influence of the base region of the vehicle that does not accurately represent flight conditions (no core engine nozzles, no SRB or core engine plumes, sting support interference, etc.). The base force corrections were applied by measuring the base pressures on the core and SRBs as discussed in Section III.D.2 and converting to body axis forces (axial force and yawing moment) by multiplying by the appropriate base areas and moment arms for the core and SRBs. Base pressure was assumed to be constant over the entire base area of each vehicle component.

\section{Roll Dependent Bias Correction}

As part of the test matrix for the Ames UPWT 11-Foot TWT test, roll sweeps were performed at $0^{\circ}$ total angle of attack at all Mach numbers. Some minor systematic biases were thereby measured, and removed during post-processing from all of the data. These systematic biases could come from very small yaw misalignments between the freestream flow and the pitch plane in which the data are acquired. The SLS-28k vehicle, with the presence of protuberances, does not present any outer mold line symmetry plane by which further data corrections could have been applied (as an example, the normal force coefficient data were not forced through zero at a total angle of attack of $0^{\circ}$ ).

Figure 9 shows an example of data from the Ames UPWT 11-Foot TWT test at Mach 1.20 with the standard facility corrections but no roll dependent bias corrections (referred to as uncorrected). The data plotted are repeat pitch sweep runs for $\phi=0^{\circ}$ (upright) and $\phi=180^{\circ}$ (inverted) at $\beta=0^{\circ}$. The figures also include roll sweep data at $\alpha=0^{\circ}$ and $\beta=0^{\circ}$. In Figure 9, the forebody coefficients plotted are in the body axes and moments are computed at the balance moment center. Looking at the pitching moment $(C L M)$ as an example, there is a clear offset between the upright and inverted runs despite being acquired at the same nominal $\alpha$ and $\beta$ conditions. It is also important to note that the Ames 
UPWT 11-Foot TWT has a symmetric contraction such that flow angularity effects are generally quite small. As such, the variations between the upright and inverted runs are not thought to be artifacts of flow angularity.
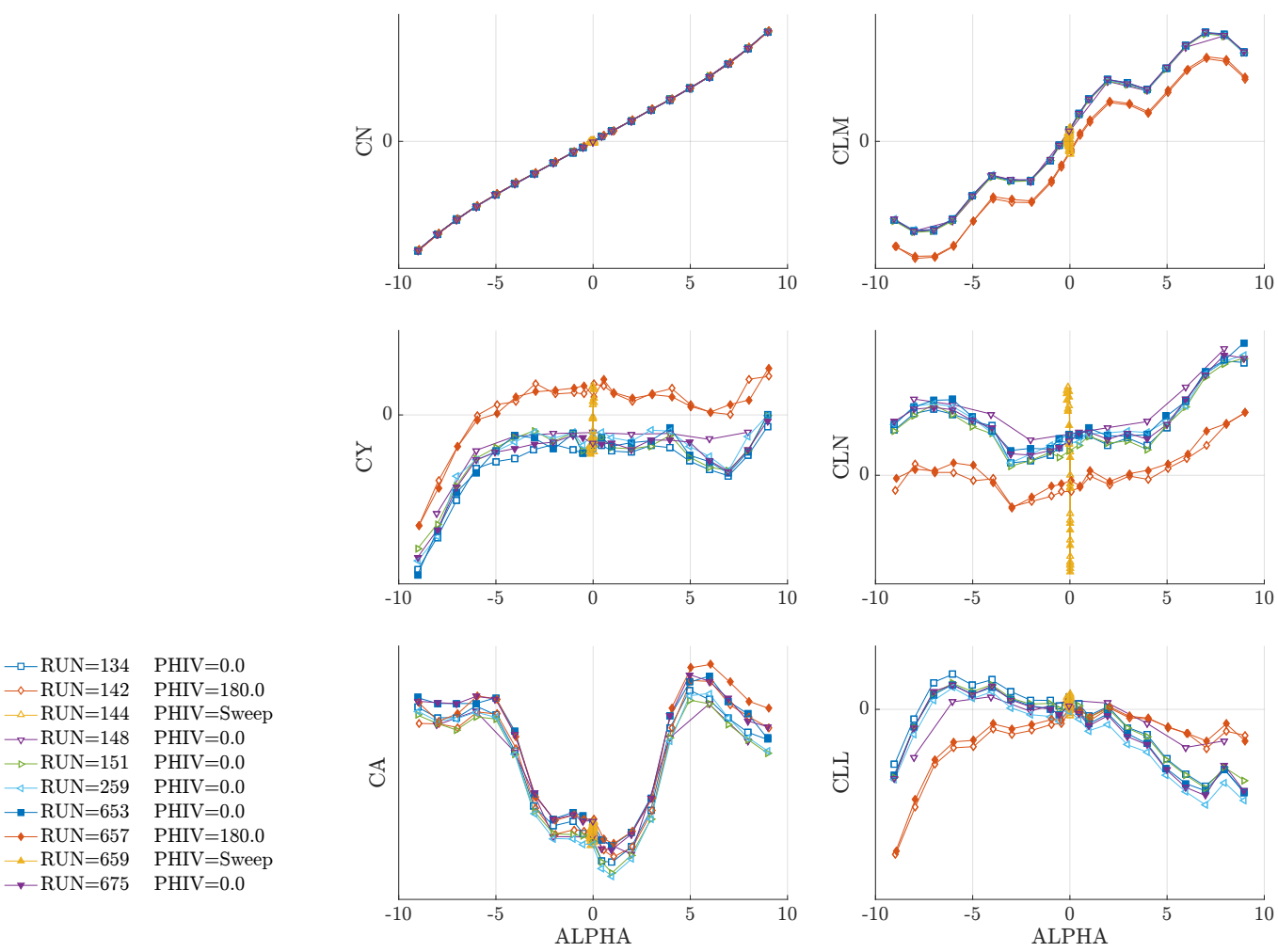

Fig. 9 Comparison of uncorrected $\phi=0^{\circ}, \phi=180^{\circ}$, and roll sweep data at Mach 1.20 and $\beta=0^{\circ}$ from the Ames UPWT 11-Foot TWT test.

To correct for these variations, roll sweeps acquired at $\alpha=0^{\circ}$ and $\beta=0^{\circ}$ are used. Figure 10 shows the two roll sweeps that are also plotted in Figure 9 (Runs $144 \& 659$ ), but are now plotted as a function of roll angle (PHIV) as opposed to pitch angle (ALPHA). As discussed previously, the SLS-28k vehicle does not have any symmetry planes. As such, the forces and moments from roll sweeps acquired at $\alpha=0^{\circ}$ and $\beta=0^{\circ}$ are not expected to be zero valued, but the forces and moments are expected to be constant valued. From Figure 10, it is clear that this is not the case and there are well defined and repeatable trends in the roll sweep data that can be used to develop a roll dependent bias correction.

To apply the correction, an average roll sweep was computed by averaging all repeat roll sweeps at $\alpha=0^{\circ}$ and $\beta=0^{\circ}$ for a given Mach number. The coefficient values calculated for average roll sweep $\left(C x_{r s}(\phi)\right)$ were then subtracted from the uncorrected coefficients $\left(C x_{U N C}(\alpha, \beta, \phi)\right)$. The mean value of the roll sweep $\left(\overline{C x_{r s}}\right)$ was then added back into the coefficients to arrive at the final corrected coefficients $\left(C x_{C O R}(\alpha, \beta, \phi)\right)$. Equation 1 summarizes these corrections, and Figure 11 shows the corrected forces and moments for Mach 1.20. Comparing pitching moment (CLM) as an example, the large offset that was seen in the uncorrected data (Figure 9) has been removed with the bias corrections in the corrected data (Figure 11).

$$
C x_{C O R}(\alpha, \beta, \phi)=C x_{U N C}(\alpha, \beta, \phi)-C x_{r s}(\phi)+\overline{C x_{r s}}
$$



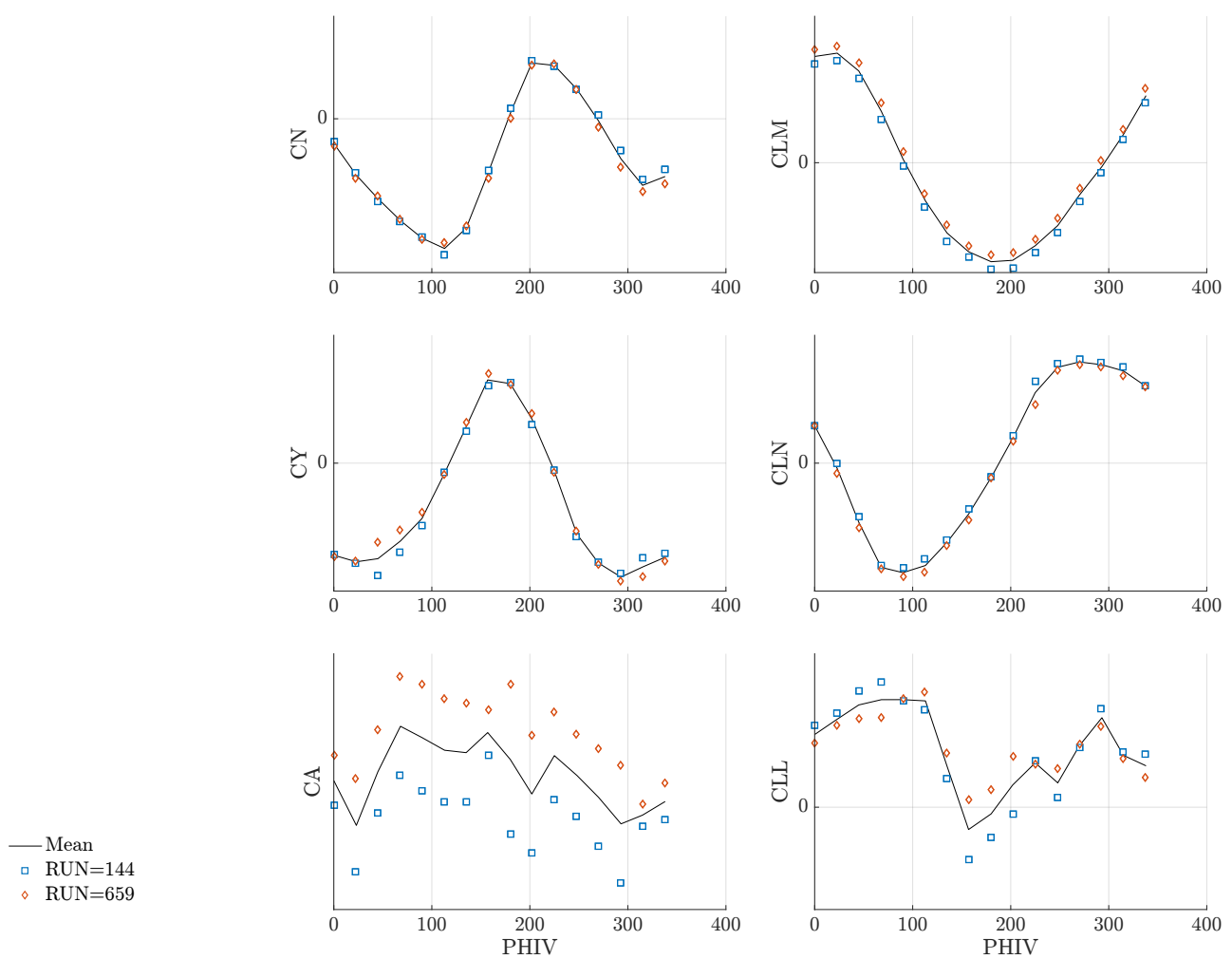

Fig. 10 Comparison of roll sweep data at Mach 1.20, $\alpha=0^{\circ}$, and $\beta=0^{\circ}$ from the Ames UPWT test.
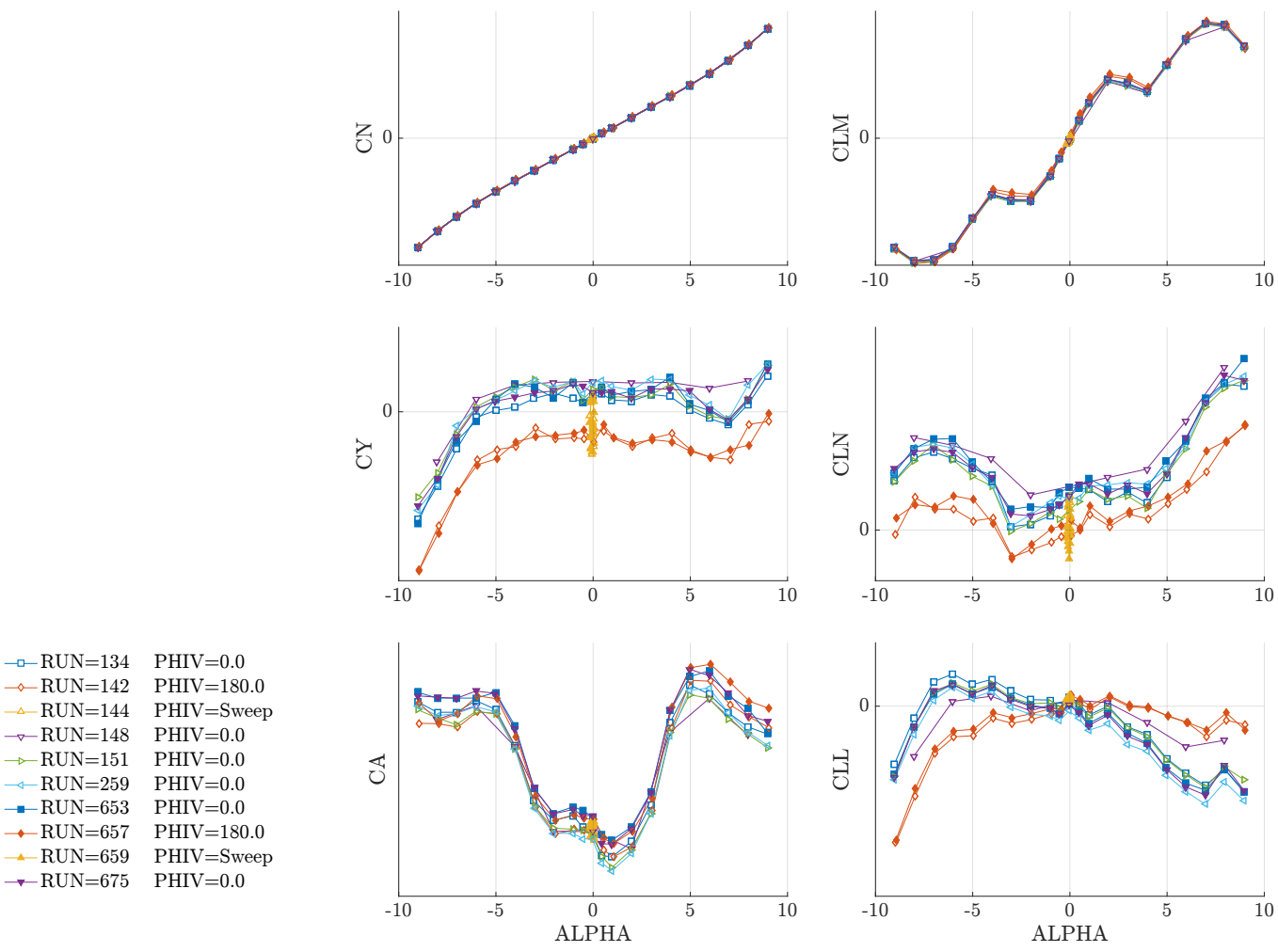

Fig. 11 Comparison of corrected data at Mach 1.20 and $\beta=0^{\circ}$ from the Ames UPWT test. 


\section{B. Response Surface Development}

Once fully corrected datasets were produced at each nominal Mach number, the data for each one of the six coefficients were interpolated to the nominal angle of attack $(\alpha)$ and sideslip $(\beta)$ breakpoints. The interpolation was performed to allow for more consistent averaging of repeat runs that were acquired at slightly different set points. A MATLAB based multivariate interpolant (built-in MATLAB function scatteredInterpolant [17]) that implements a natural neighbor interpolation scheme was computed for each coefficient and Mach number. The response surface was then interrogated at each $(\alpha, \beta)$ breakpoint combination for delivery in the final look-up tables.

As an example of the interpolated database surfaces, Figures 12 through 14 show the wind tunnel data (colored symbols on the lower contour plot with black outlines) and the multivariate interpolant (colored profile on upper plot and colored surface on lower plot). The response surfaces shown here are at Mach 1.20 and are plotted as a function of $\alpha$ and $\beta$. Comparing the raw wind tunnel data (colored symbols on the lower plots) to the final response surface, it can be seen that the multivariate interpolant represents the input data well as is required. This is true for both simple surfaces such as the normal force (Fig. 12) as well as the more complex surfaces such as rolling moments (Fig. 14).

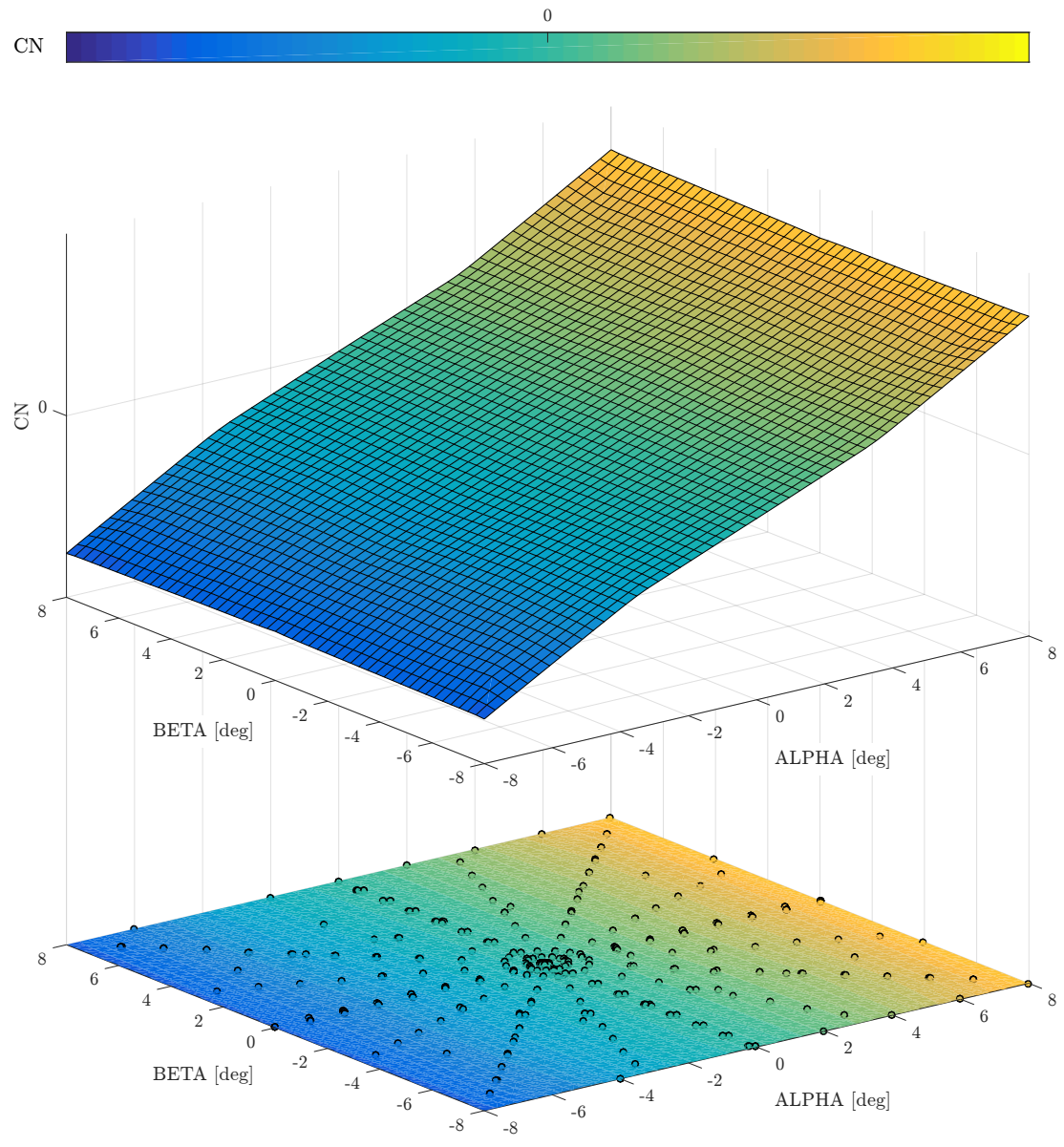

Fig. 12 Normal force coefficient $(C N)$ response surface for Mach 1.20. Developed using Ames UPWT data. 


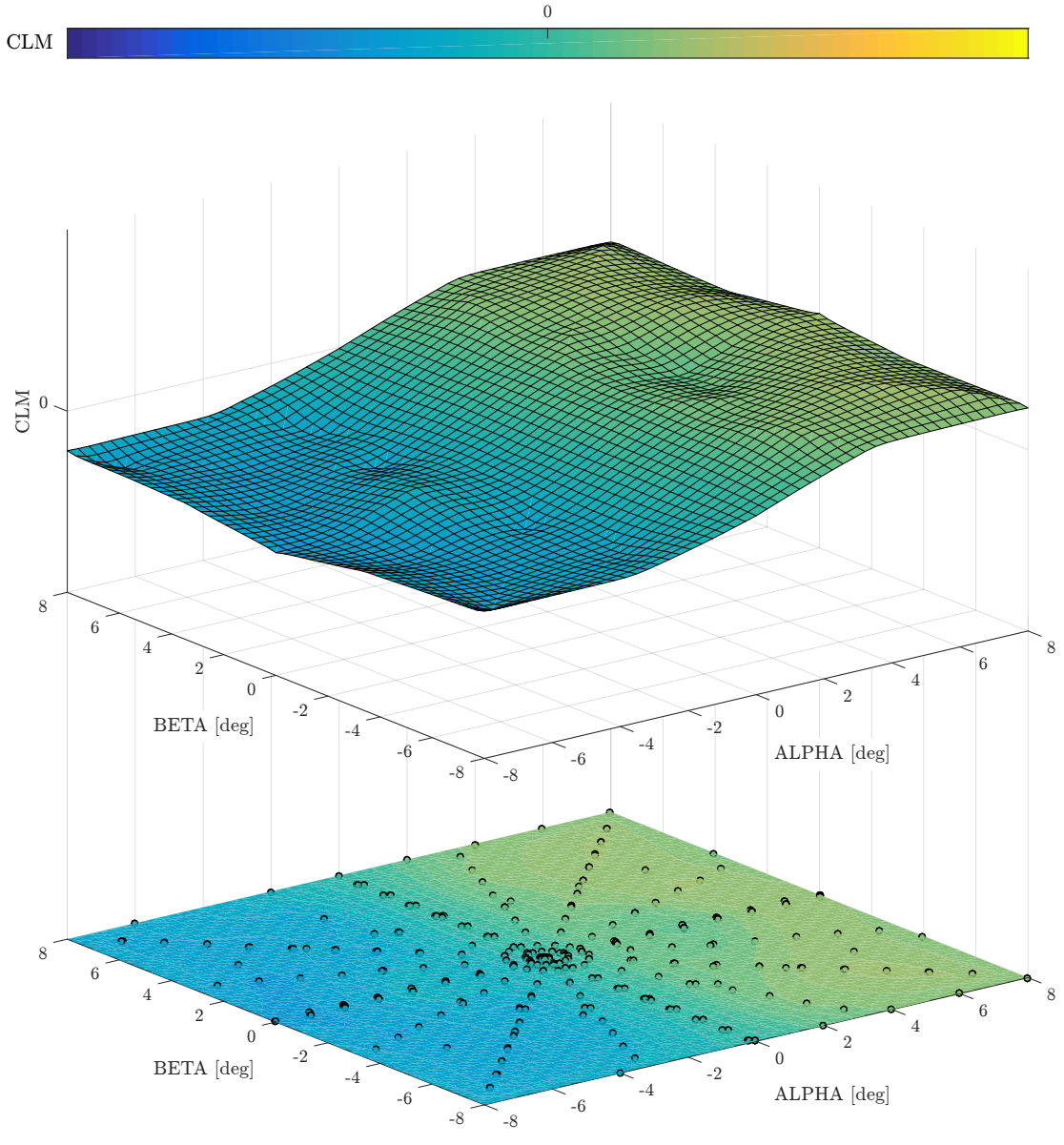

Fig. 13 Pitching moment coefficient $(C L M)$ response surface computed at the balance moment center for Mach 1.20. Developed using Ames UPWT data. 


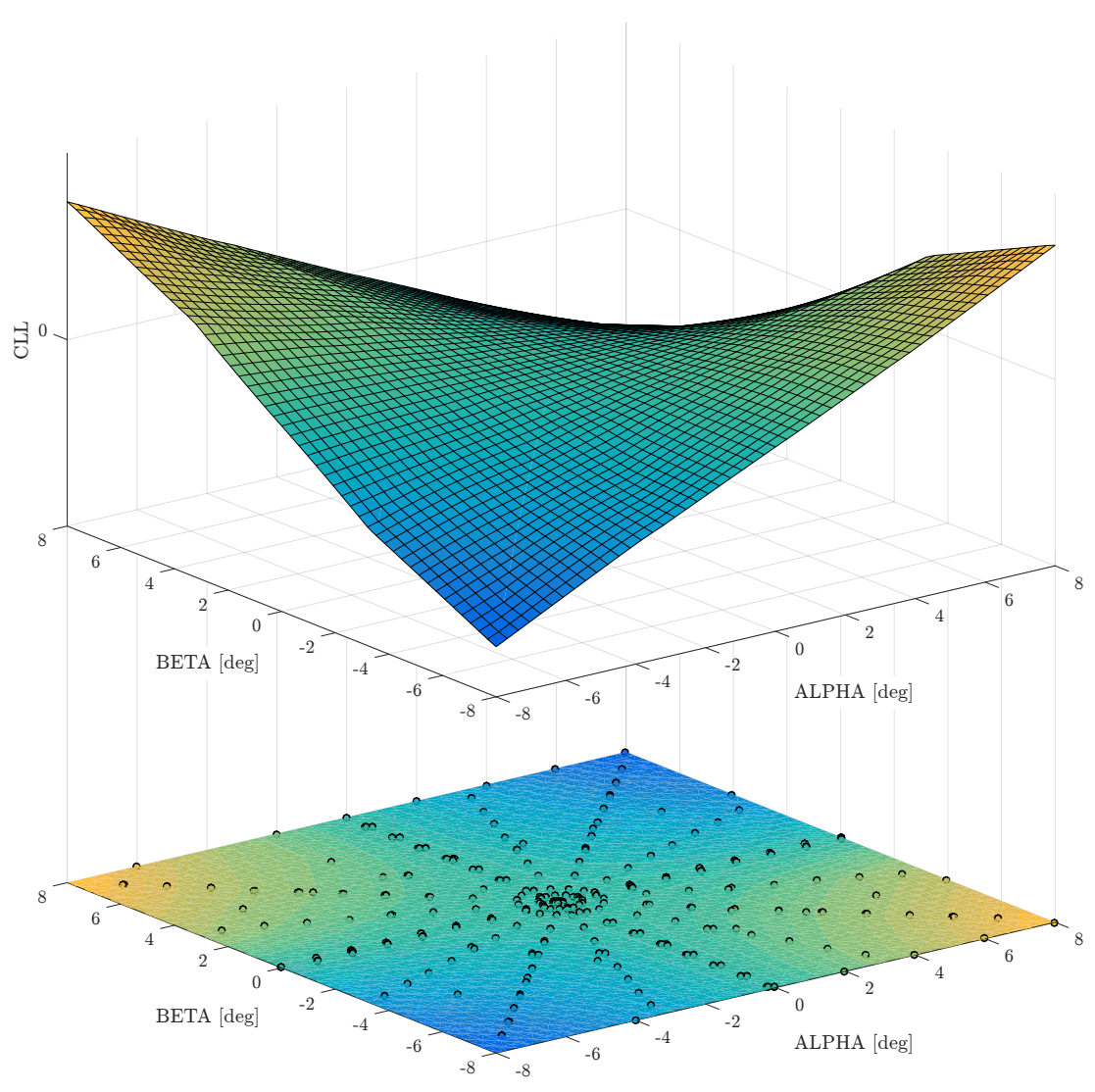

Fig. 14 Rolling moment coefficient $(C L L)$ response surface computed at the balance moment center for Mach 1.20. Developed using Ames UPWT data.

Response surface development was not limited to using the experimental wind tunnel database as an input. The CFD solutions developed for both the wind tunnel test conditions and flight conditions had sufficient $\alpha$, $\beta$, and Mach resolution to develop response surfaces using the techniques described above for the experimental data. In the sections that follow, response surfaces have been developed for the experimental wind tunnel data, the CFD solutions at wind tunnel conditions, and CFD at flight conditions. Comparisons between the three databases are made by querying the response surfaces as opposed to the source data.

\section{WT to CFD Comparisons}

As discussed previously, CFD simulations were run at the wind tunnel test conditions. Response surfaces for the wind tunnel dataset and CFD simulations at wind tunnel conditions were developed using the methods outlined in Section V.B, and these response surfaces were then queried at matching conditions to make quantitative assessments of the data quality. In the figures that follow, the response surfaces for each coefficient are plotted at scales relevant to each coefficient, but the CFD and WT surfaces are plotted on the same scale. For example, the normal force surfaces (Figs. 15(a) \& 15(b)) are both plotted on the same scale, but this scale is not the same as the scale used for side force (Figs. 19(a) \& 19(b)). When differences are presented between the two datasets, all of the forces $(\triangle C N, \triangle C Y$, and $\triangle C A$ ) are plotted on the same scale, and all of the moments ( $\triangle C L L, \triangle C L M$, and $\triangle C L N)$ are plotted on the same scale to allow for qualitative comparison of the different forces and moments.

The normal force results are compared in Figures $15 \& 16$. In Figure 15, the response surfaces are compared at Mach 1.20 as a function of $\alpha$ and $\beta$. Figure 15(a) shows the response surface computed from the CFD data at wind 
tunnel conditions, and Figure 15(b) shows the response surface computed from the wind tunnel data (note that this is the same figure as Figure 12 and is included here for comparison purposes). Recall that these two figures are plotted on the same scales (both the vertical axis and contour coloring) such that direct comparisons can be made. The symbols (colored circles with black outlines) on the lower plot indicate the locations as well as the value of the input data. Note that the CFD data are more sparse than the wind tunnel data, but were computed at the matching setpoints. Visually, the two response surfaces compare well and have similar slopes and magnitudes. Figure 15(c) is a direct subtraction of the CFD response surface from the wind tunnel response surface where the vertical axis limits and color map $(\Delta C N)$ have a range of approximately $10 \%$ of the $C N$ response surface plot range. The color map has been defined such that red values indicate that the wind tunnel data are higher valued $(\mathrm{CFD}<\mathrm{WT})$, blue values indicate that the CFD data are higher valued (CFD > WT), and white indicates that the two response surfaces have the same value (CFD = WT). From the direct subtraction, it can be seen that the CFD compares well to the experimental values in general. At negative values of $\alpha$, the magnitude of the CFD is less than the wind tunnel (more negative), and at positive values of $\alpha$, the magnitude of the CFD is larger than the wind tunnel (more positive) indicating that the CFD has a slightly larger slope in $C N$ relative to $\alpha$ as compared to the wind tunnel.

Figure 16 compares point data queried from the response surfaces and plots the differences as a function of $\alpha$ (Fig. 16(a)), $\beta$ (Fig. 16(b)), and Mach number (Fig. 16(c)). These plots are used to look for trends across the entire database. The $\triangle C N$ data are seen to be scattered around zero indicating that there is no significant bias between the two datasets. Looking at Figures 16(b) \& 16(c), no discernible trends can be seen in the differences. Looking at Figure 16(a), there is a negative trend in $\triangle C N$ as a function of $\alpha$ consistent with the slightly larger slope identified at Mach 1.20 (Fig. 15(c)). This trend is very weak, however, and these results generally indicate good normal force agreement between the CFD and WT datasets.

Similar plots for pitching moment (CLM and $\triangle C L M)$ computed at the experimental balance moment center can be found in Figures $17 \& 18$. Of the six forces and moments, pitching moment generally has the largest discrepancies between CFD and the WT databases. Starting with the response surfaces in Figures 17(a) \& 17(b), the differences in the general behavior of the response surfaces can be seen clearly, especially around $\alpha= \pm 4^{\circ}$. These differences become more apparent when looking at the difference of the surfaces at Mach 1.20 (Fig. 17(c)). The vertical axis limits and color map for Fig. 17(c) ( $\triangle C L M)$ have a range of approximately 50\% of the CLM response surface plot range. Looking at the point comparisons in Figure 18, the trend in $\alpha$ (Fig. 18(a)) is seen to be consistent across the entire database with the scatter about zero highest near Mach 1.00 (Fig. 18(c)). The exact cause of the differences between pitching moment for the CFD and WT datasets has not been identified, but the authors hypothesize that the discrepancies most likely originate from the complex flow near the SRB forward attach hardware. Around the forward attach hardware, there is a forced boundary layer transition on the wind tunnel model SRB nose cones that is modeled as fully turbulent in the CFD, there are differences in the level of fidelity between the CFD and WT resulting from the practical limitations of scaled model fabrication, and there are potentially contributions from the RANS modeling and grid refinement in the wake of the attachment hardware that could result in differences downstream of the attach point.

Comparisons of side force (Figures $19 \& 20$ ), yawing moment (Figures $21 \& 22$ ), and rolling moment (Figures 25 \& 26) show good agreement. Recall that $\triangle C Y$ is plotted on the same scales as $\triangle C N$, and $\triangle C L L$ and $\triangle C L N$ are plotted on the same scales as $\triangle C L M$. With this in mind, it can be concluded that the differences for normal and side force have similar magnitudes and both generally agree well. The differences computed for $\triangle C L L$ and $\triangle C L N$ are much smaller than those seen for $\triangle C L M$, which is not unexpected considering the SRB layout on the vehicle that results in higher pitching moment sensitivities. No discernible trends are seen in Mach, $\alpha$, or $\beta$ for $\triangle C Y, \triangle C L L$, or $\triangle C L N$.

Comparing the response surfaces for axial force (CA), differences in the surfaces can be seen around $\alpha=0^{\circ}$ with the CFD (Fig. 23(a)) exhibiting a decrease in the nominal values while the wind tunnel data (Fig. 23(b)) remains fairly constant across the entire $\alpha$ and $\beta$ space. This difference near $\alpha=0^{\circ}$ can be seen more clearly in the direct subtraction of the two datasets (Fig. 23(c)). The direct subtraction also shows an offset between the two datasets with the wind tunnel data having a higher value (indicated by the red contour levels) for all points at Mach $=1.20$. The vertical axis limits and color map for Fig. 23(c) ( $\triangle C A$ ) have a range of approximately $20 \%$ of the $C A$ response surface plot range. Looking at the point comparisons in Figure 24, a well defined trend as a function of Mach number (Fig. 24(c)) can be seen with the largest discrepancies peaking around Mach $=1.60$. The offsets between the CFD and wind tunnel data are still under investigation, but potential sources of this difference include the base force correction in the wind tunnel test data or differences in transition to a turbulent boundary layer (fully turbulent in CFD, forced transition in the wind tunnel) resulting in different boundary layer growth and skin friction along the length of the vehicle. The peak in the difference around $\alpha=0^{\circ}$ can be seen in Figure 24(a) indicating that the trend as a function of $\alpha$ seen at Mach 1.20 (Fig. 23(c)) exists for the entire database. No discernible trends are seen as a function of $\beta$. 


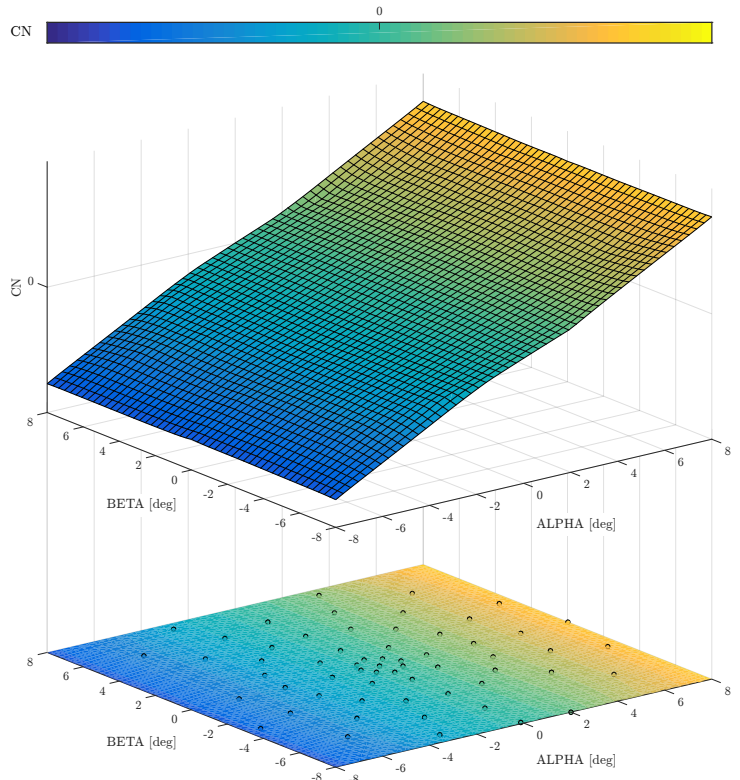

(a) CFD at Wind Tunnel Conditions

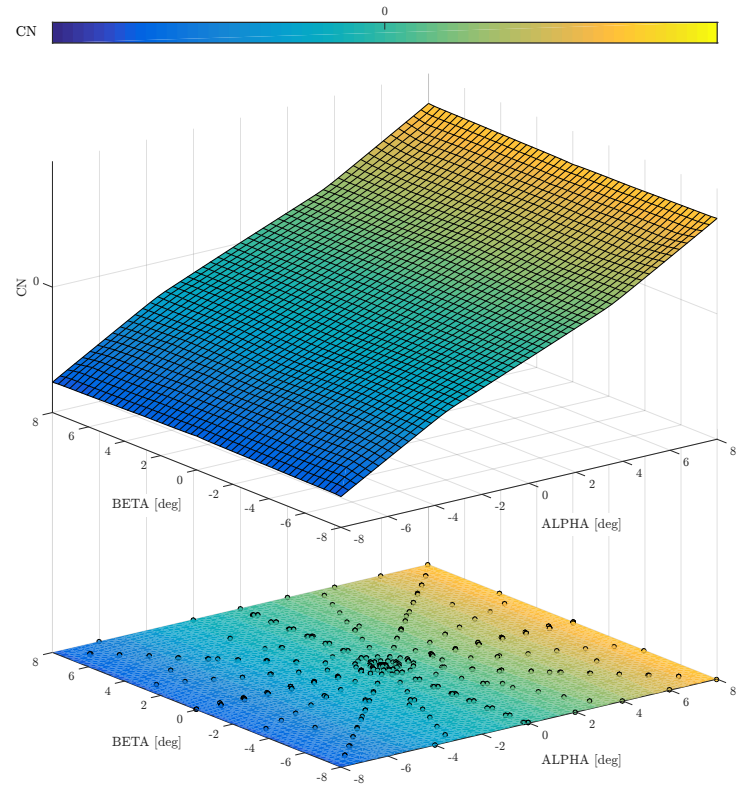

(b) Wind Tunnel Database

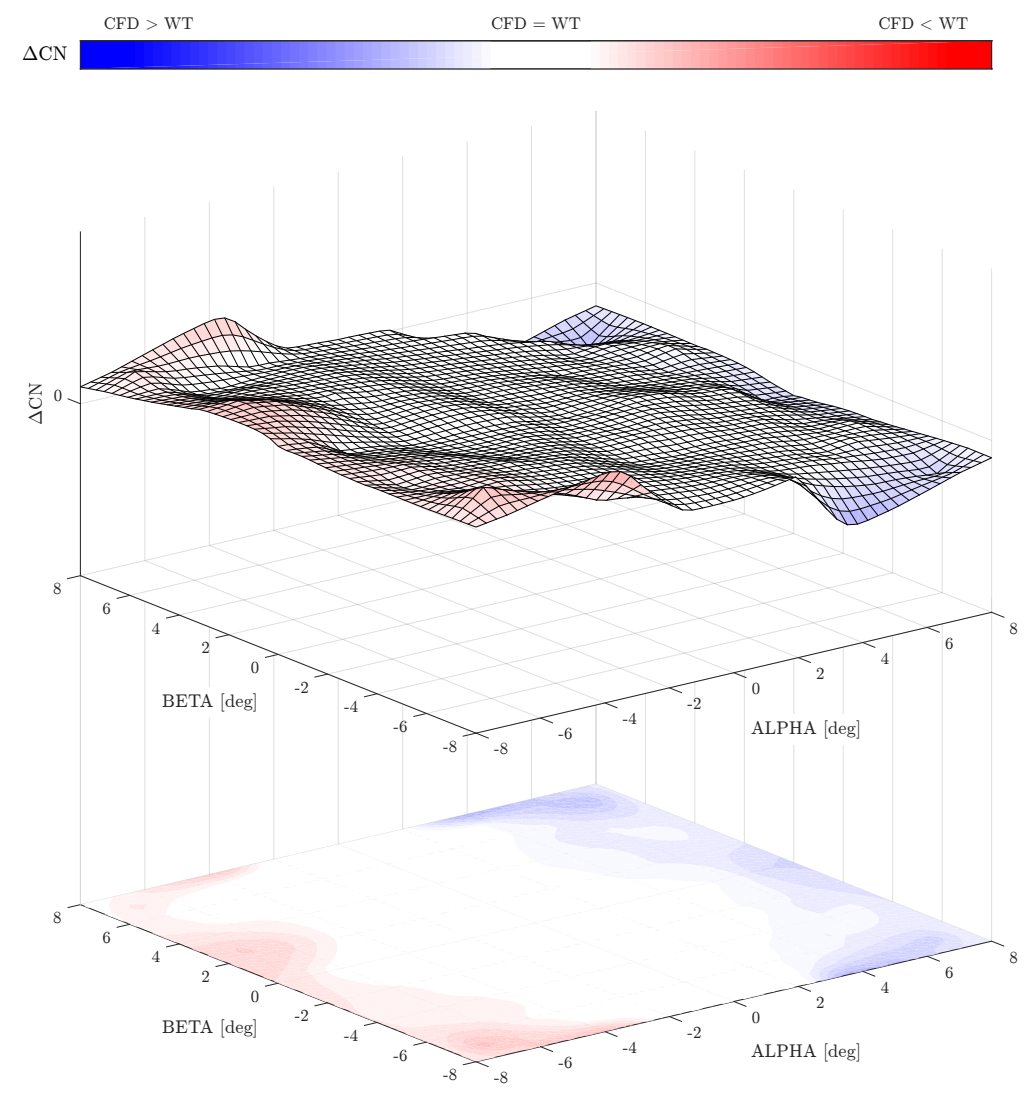

(c) Difference Between Wind Tunnel Database and CFD Solution at Wind Tunnel Conditions

Fig. 15 Comparison of CFD and wind tunnel based normal force coefficient ( $C N$ and $\triangle C N)$ response surfaces at Mach 1.20. 


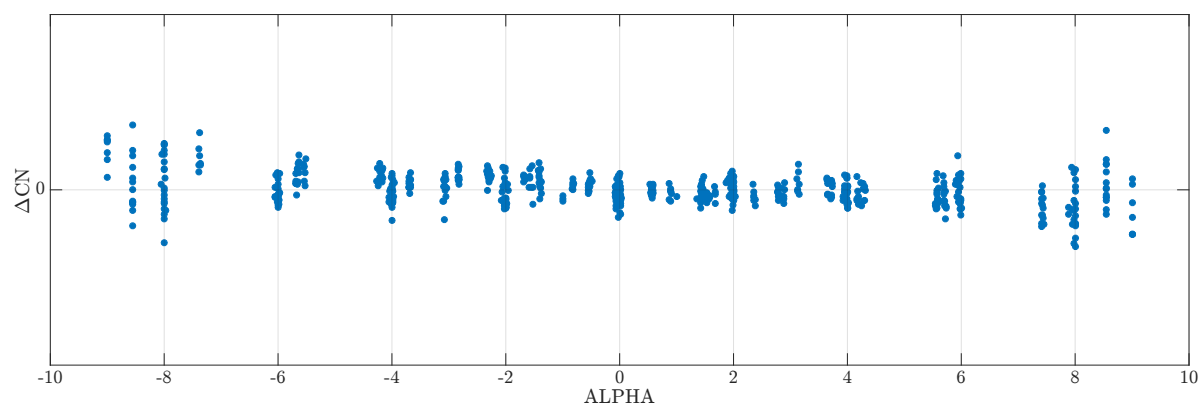

(a) Plotted as a Function of Alpha

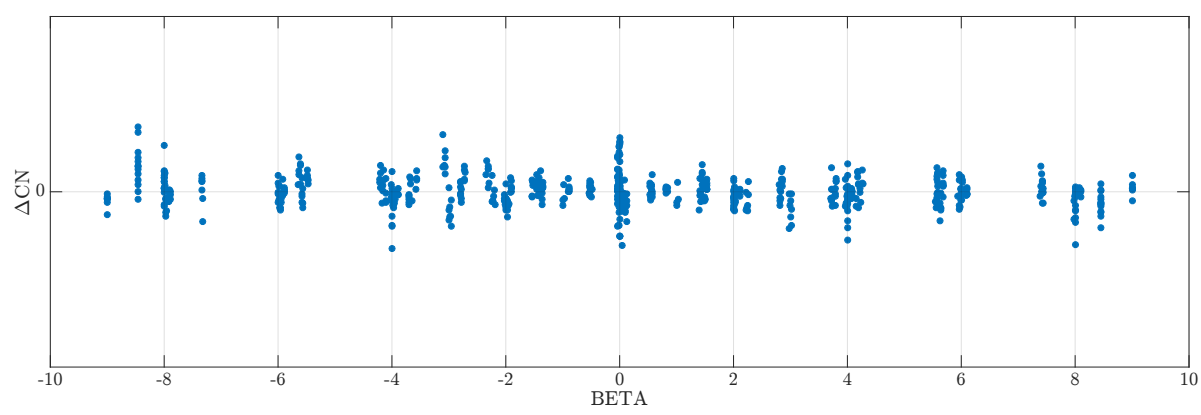

(b) Plotted as a Function of Beta

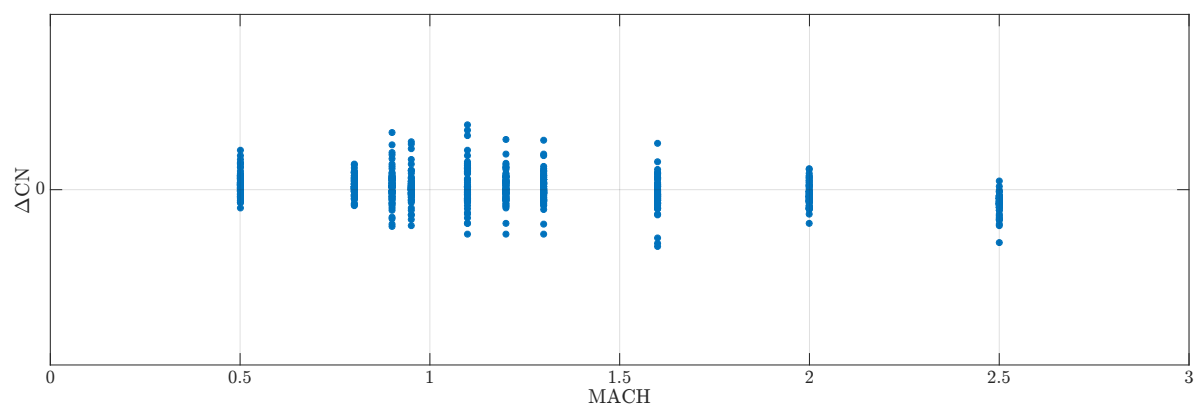

(c) Plotted as a Function of Mach

Fig. 16 Difference of wind tunnel and CFD at wind tunnel conditions based normal force coefficient $(\triangle C N)$ values. 


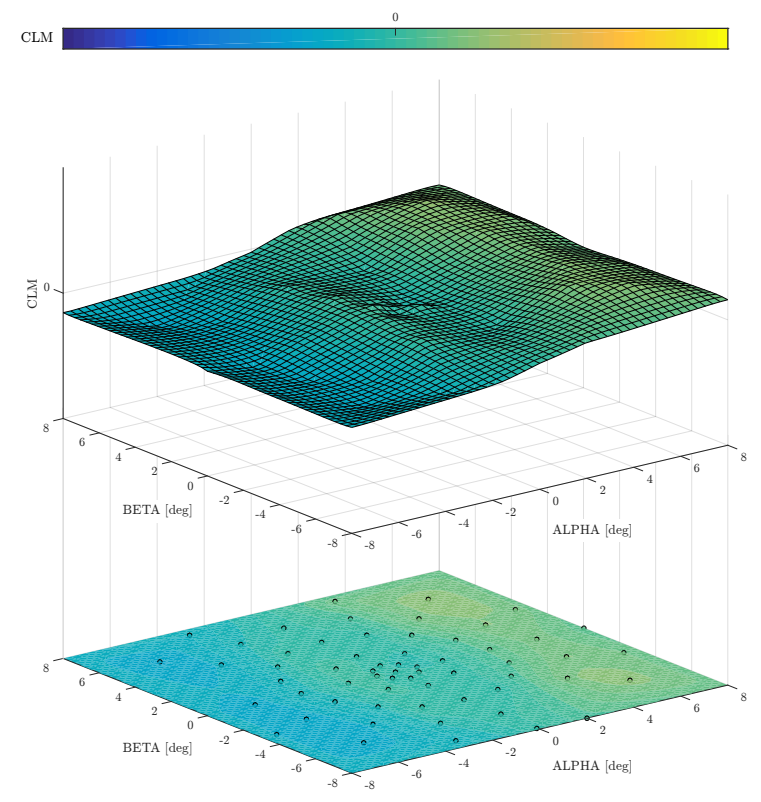

(a) CFD at Wind Tunnel Conditions

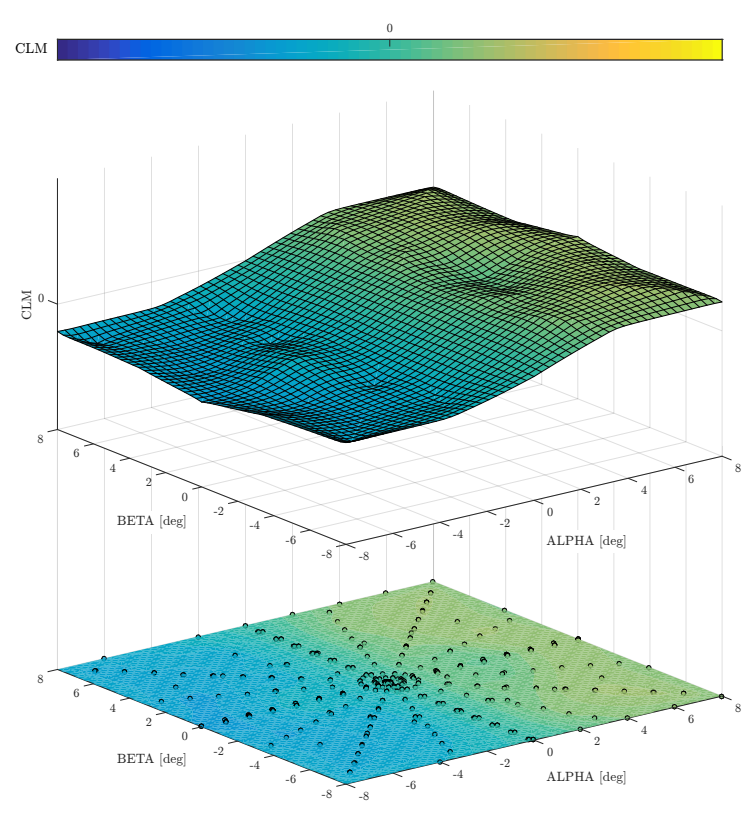

(b) Wind Tunnel Database

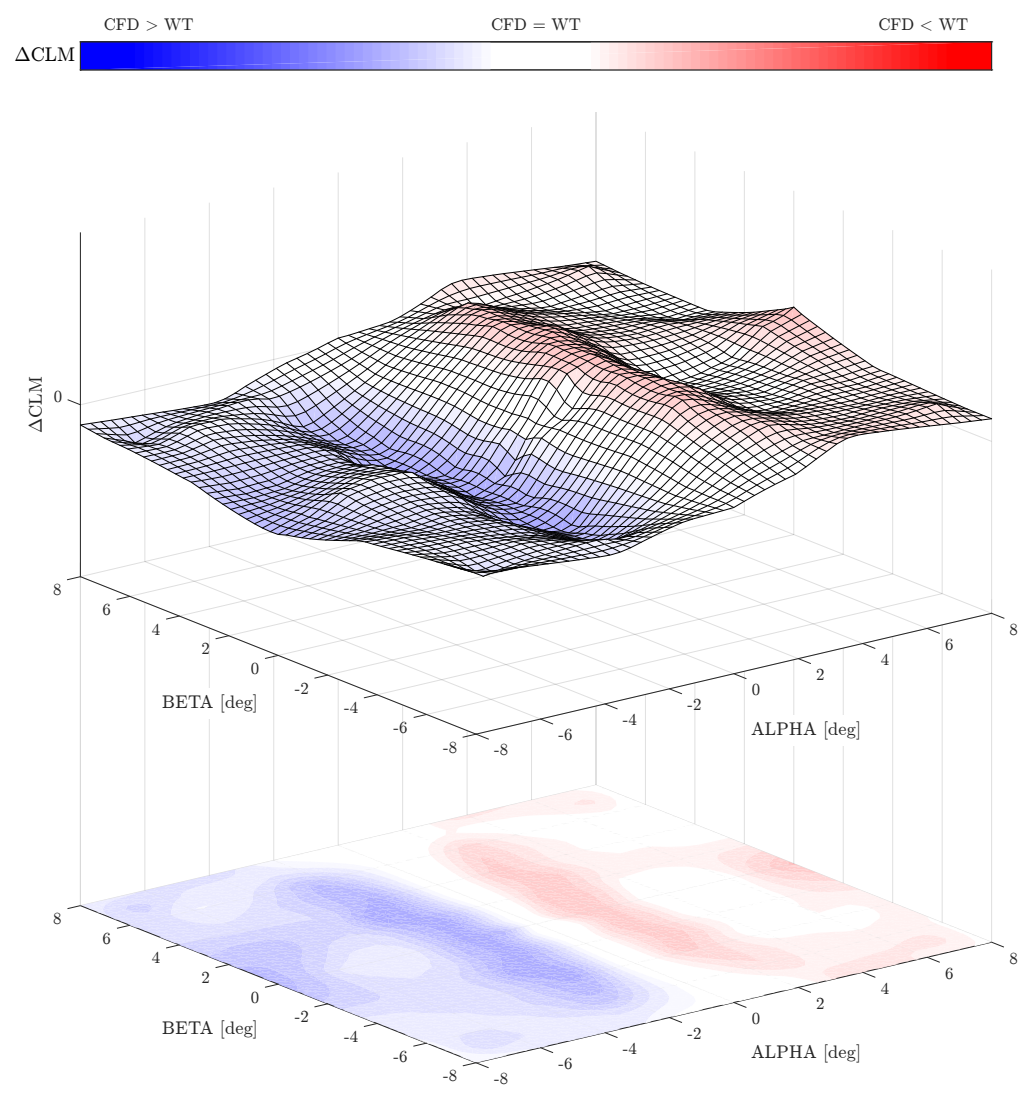

(c) Difference Between Wind Tunnel Database and CFD Solution at Wind Tunnel Conditions

Fig. 17 Comparison of CFD and wind tunnel based pitching moment coefficient (CLM and $\triangle C L M$ ) response surfaces at Mach 1.20. 


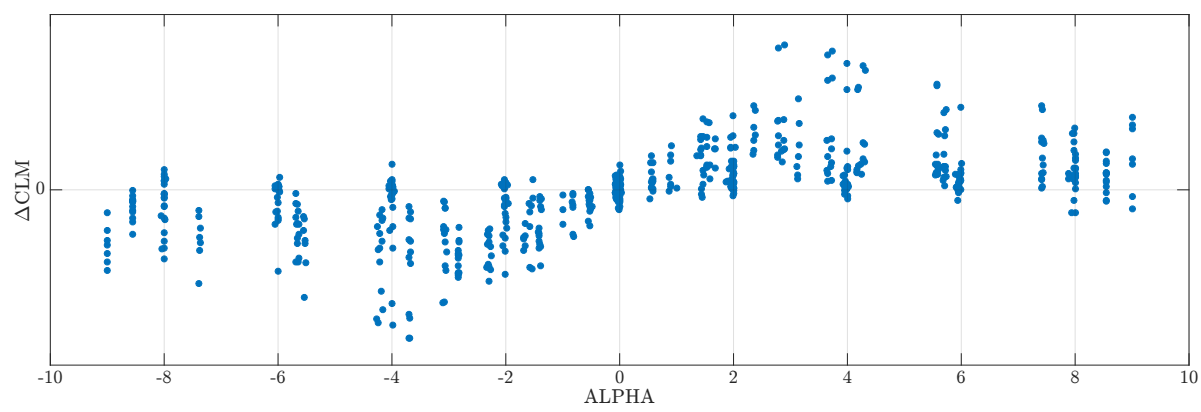

(a) Plotted as a Function of Alpha

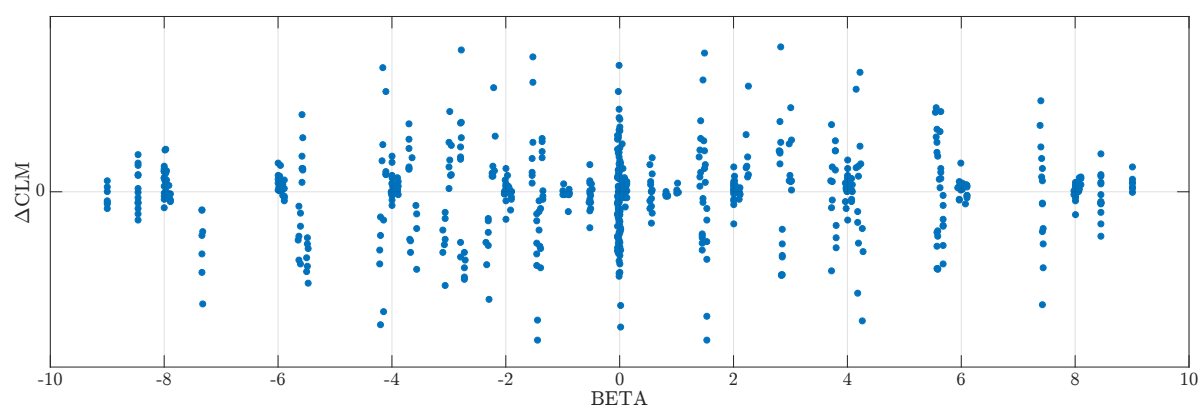

(b) Plotted as a Function of Beta

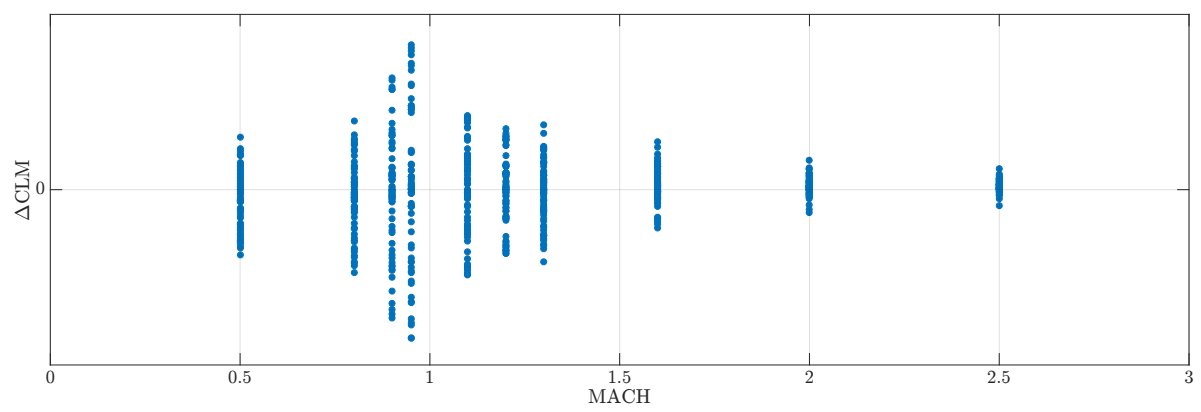

(c) Plotted as a Function of Mach

Fig. 18 Difference of wind tunnel and CFD at wind tunnel conditions based pitching moment coefficient $(\triangle C L M)$ values. 


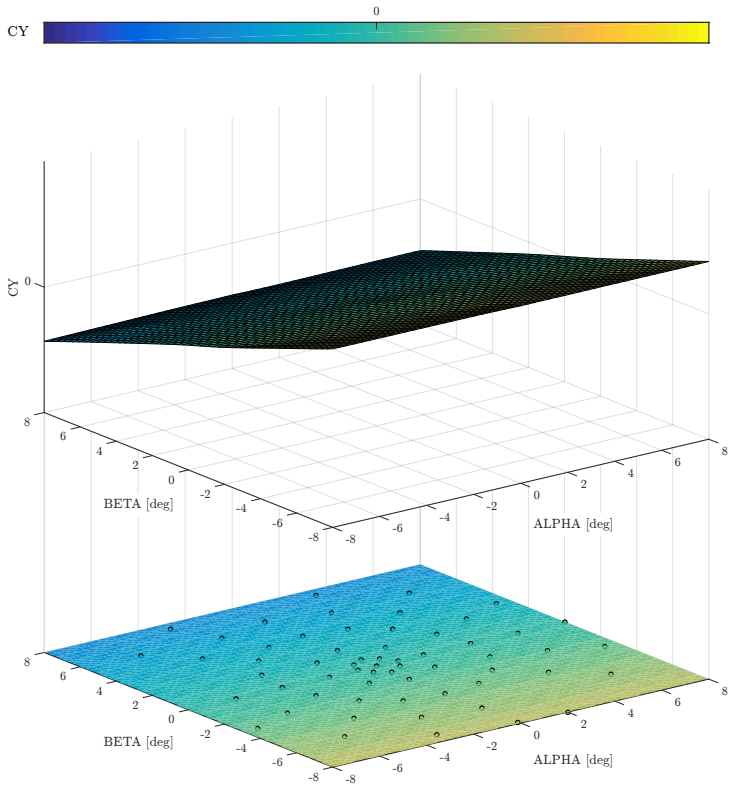

(a) CFD at Wind Tunnel Conditions

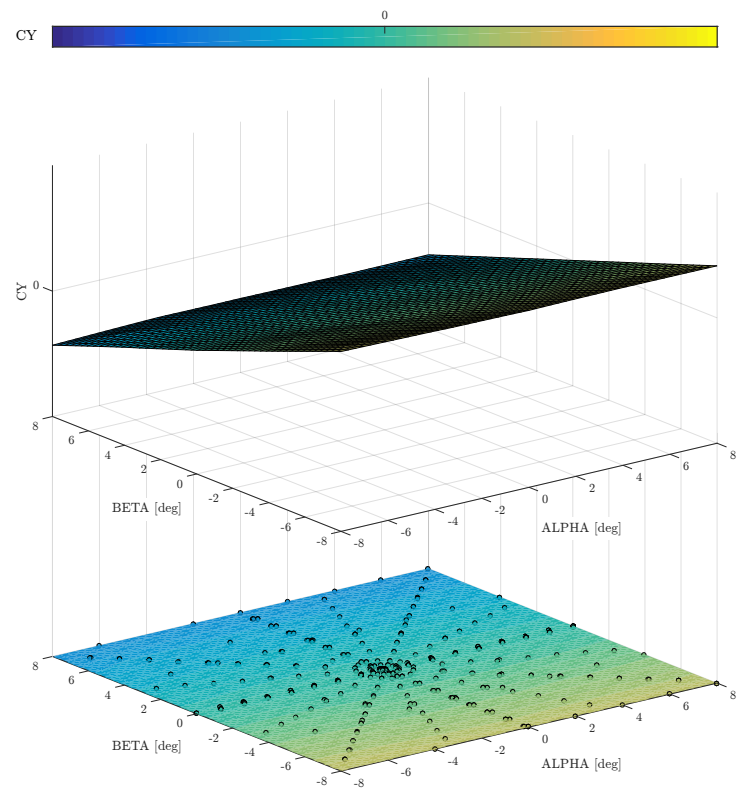

(b) Wind Tunnel Database

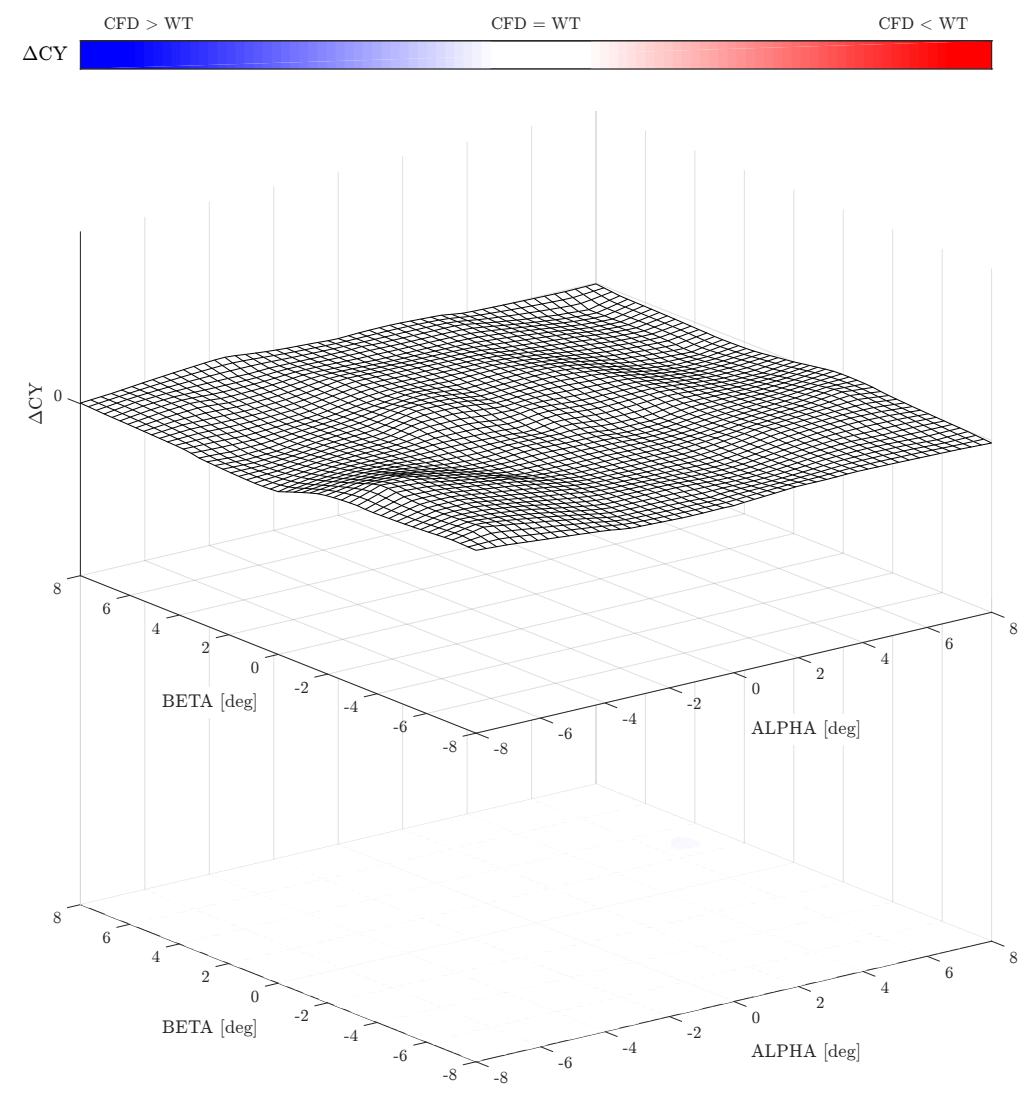

(c) Difference Between Wind Tunnel Database and CFD Solution at Wind Tunnel Conditions

Fig. 19 Comparison of CFD and wind tunnel based side force coefficient ( $C Y$ and $\triangle C Y$ ) response surfaces at Mach 1.20. 


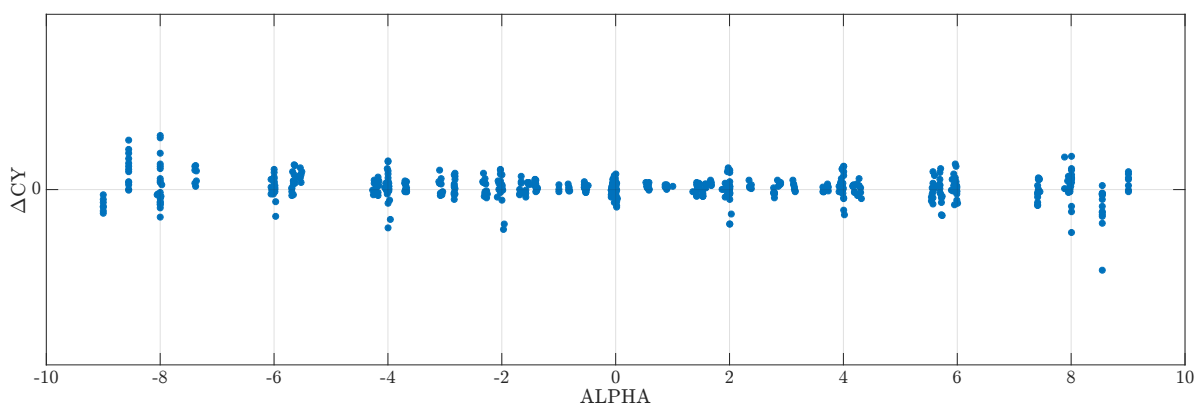

(a) Plotted as a Function of Alpha

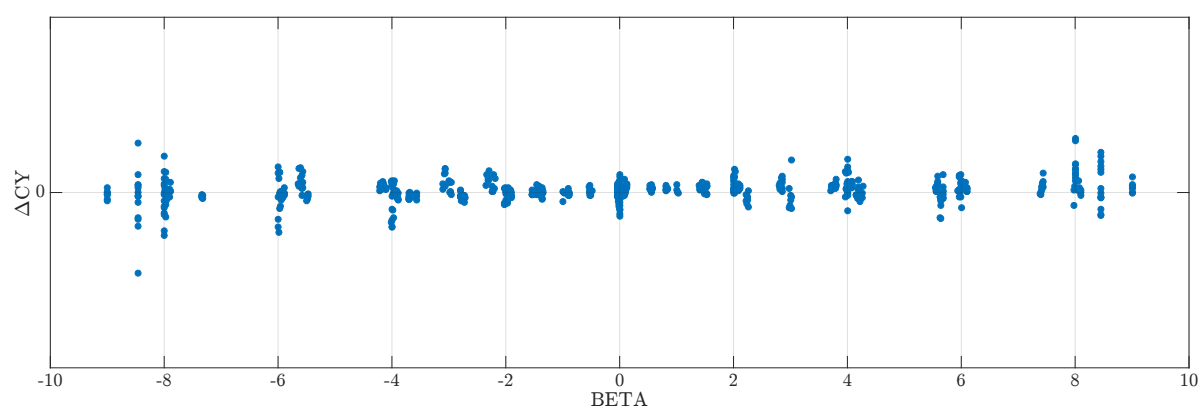

(b) Plotted as a Function of Beta

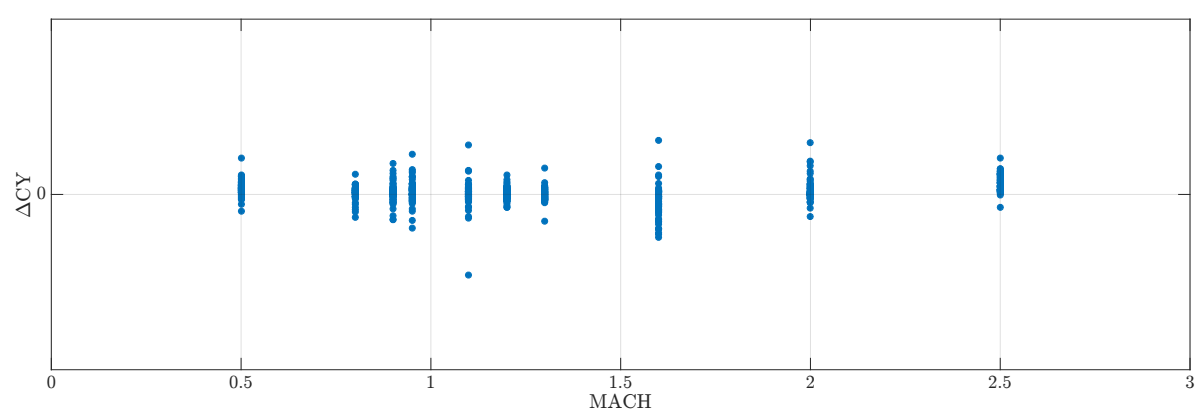

(c) Plotted as a Function of Mach

Fig. 20 Difference of wind tunnel and CFD at wind tunnel conditions based side force coefficient $(\triangle C Y)$ values. 


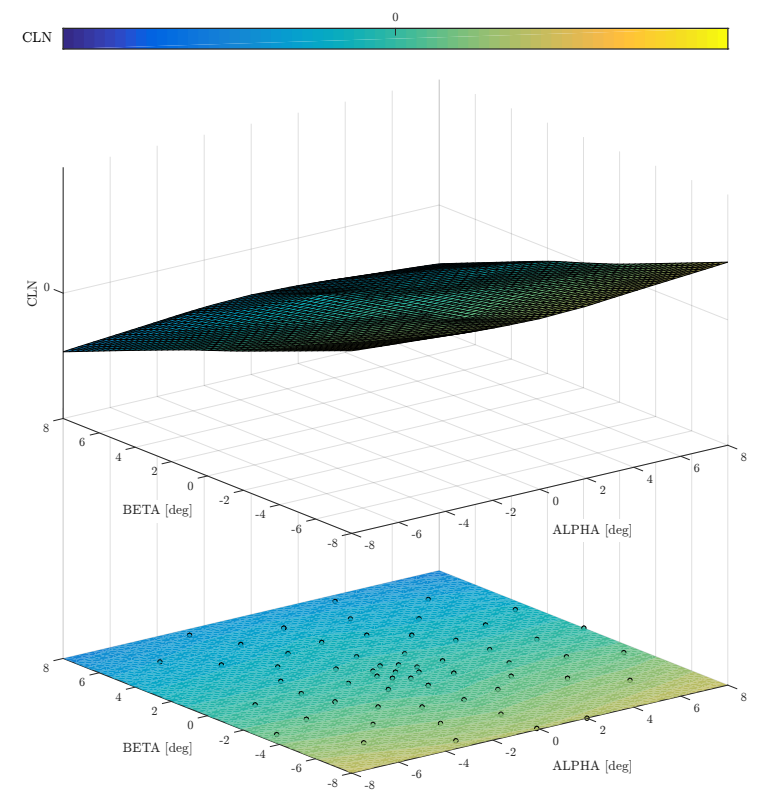

(a) CFD at Wind Tunnel Conditions

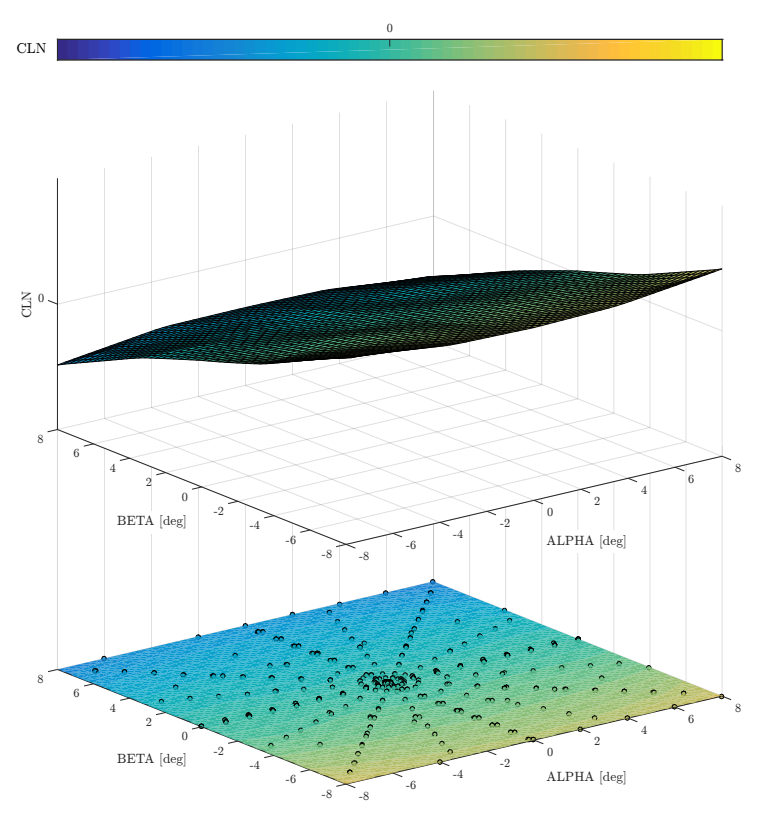

(b) Wind Tunnel Database

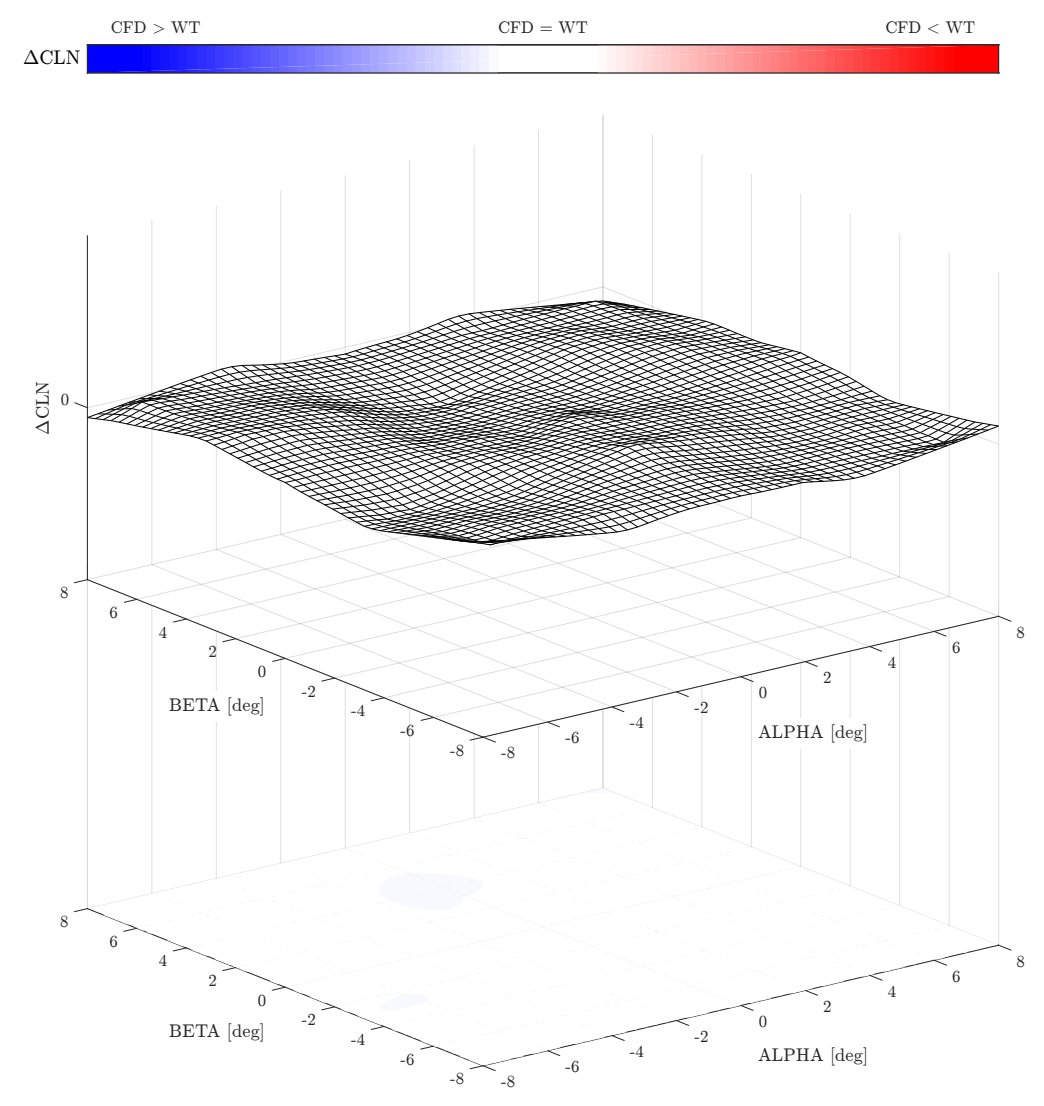

(c) Difference Between Wind Tunnel Database and CFD Solution at Wind Tunnel Conditions

Fig. 21 Comparison of CFD and wind tunnel based yawing moment coefficient ( $C L N$ and $\triangle C L N)$ response surfaces at Mach 1.20. 


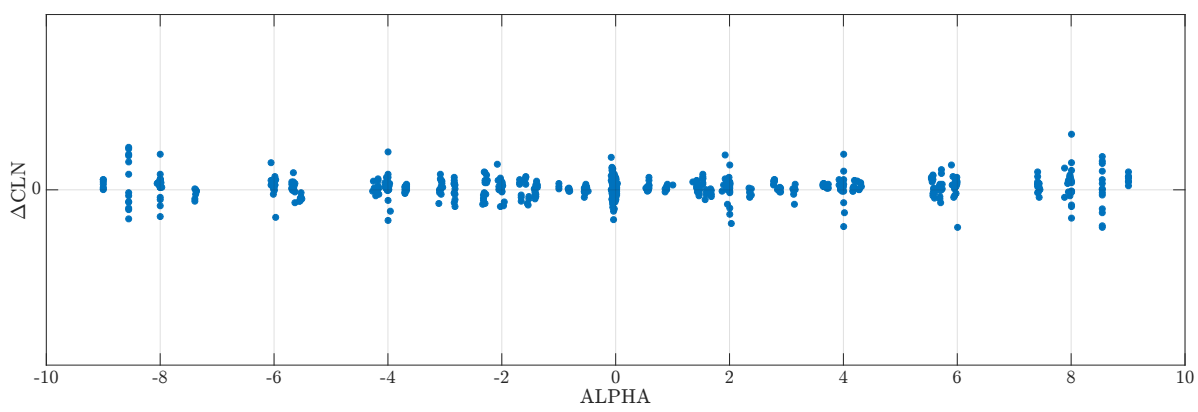

(a) Plotted as a Function of Alpha

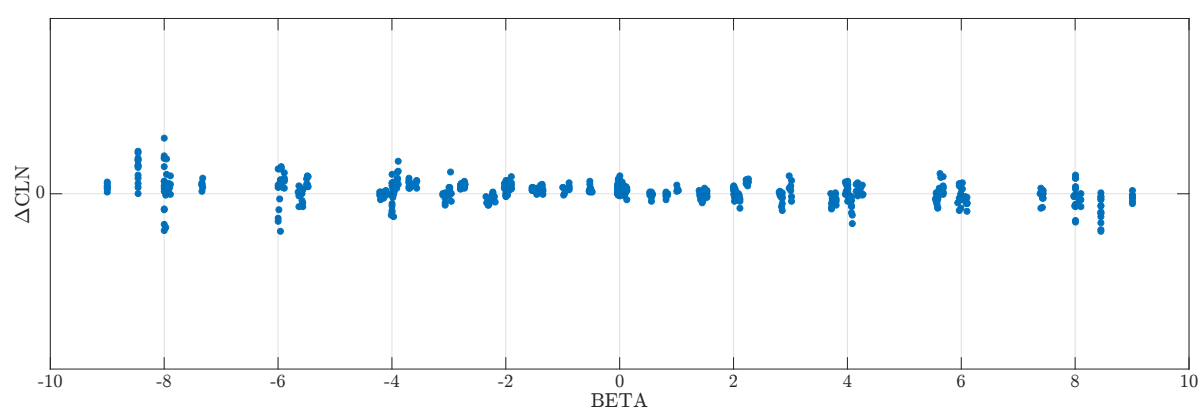

(b) Plotted as a Function of Beta

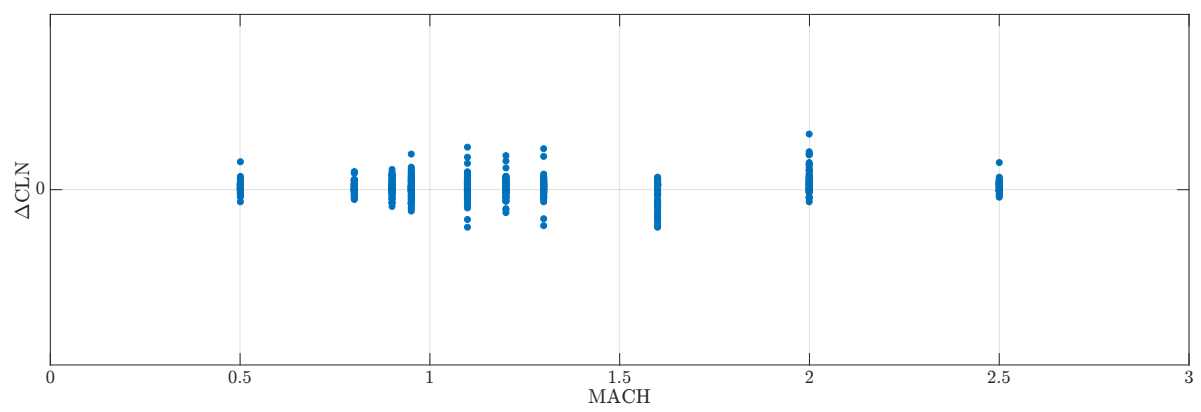

(c) Plotted as a Function of Mach

Fig. 22 Difference of wind tunnel and CFD at wind tunnel conditions based yawing moment coefficient $(\triangle C L N)$ values. 


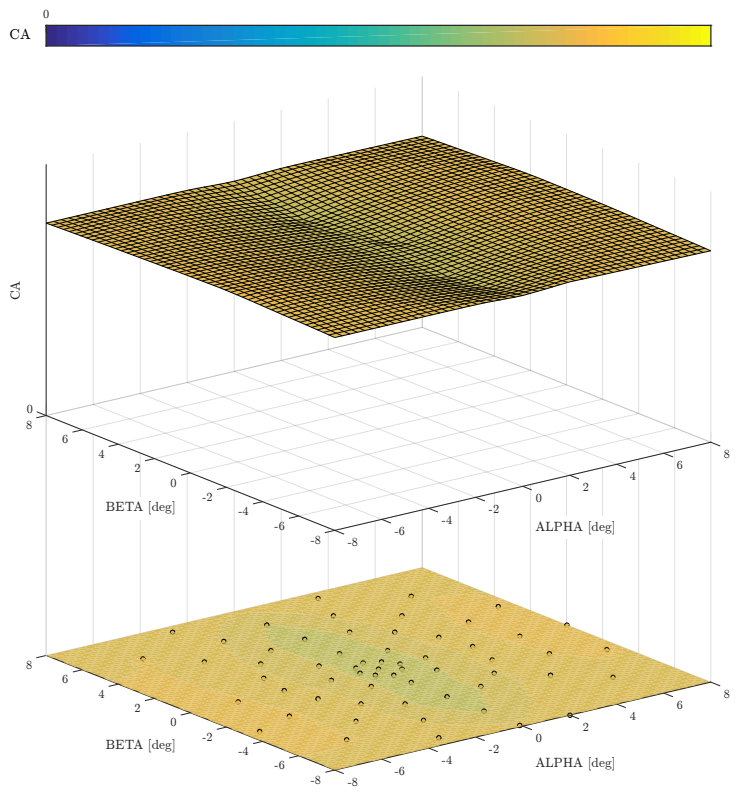

(a) CFD at Wind Tunnel Conditions

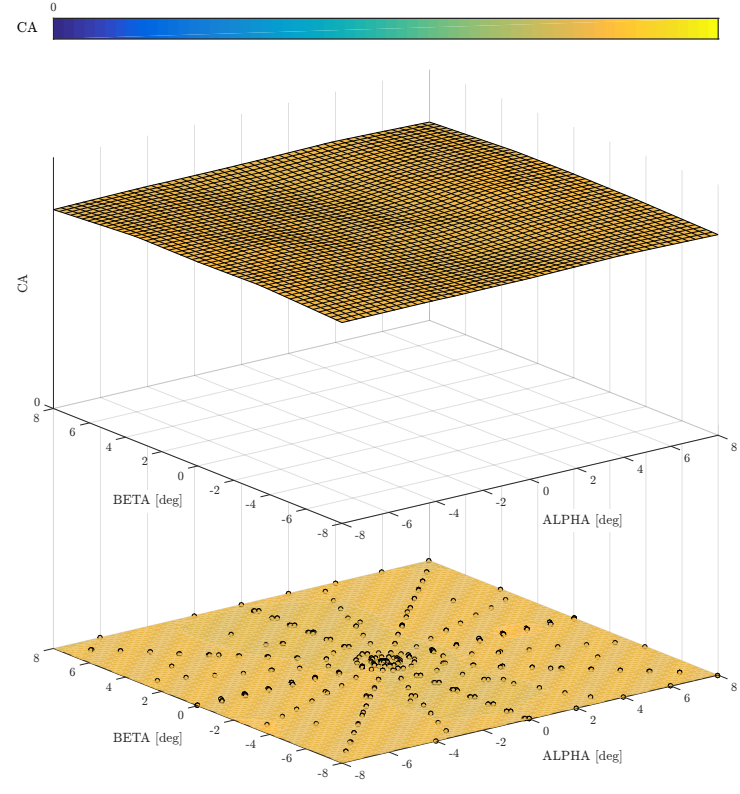

(b) Wind Tunnel Database

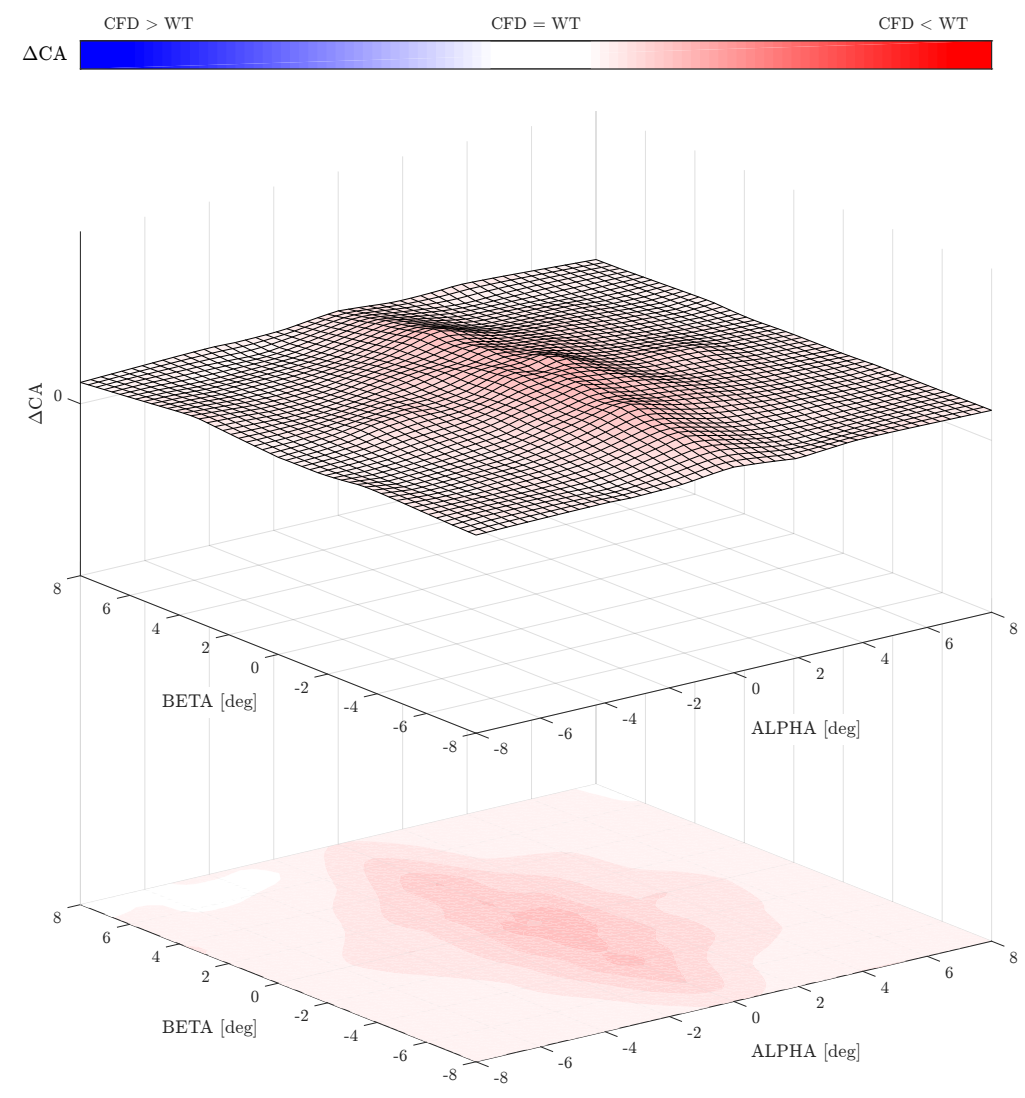

(c) Difference Between Wind Tunnel Database and CFD Solution at Wind Tunnel Conditions

Fig. 23 Comparison of CFD and wind tunnel based axial force coefficient ( $C A$ and $\triangle C A$ ) response surfaces at Mach 1.20. 


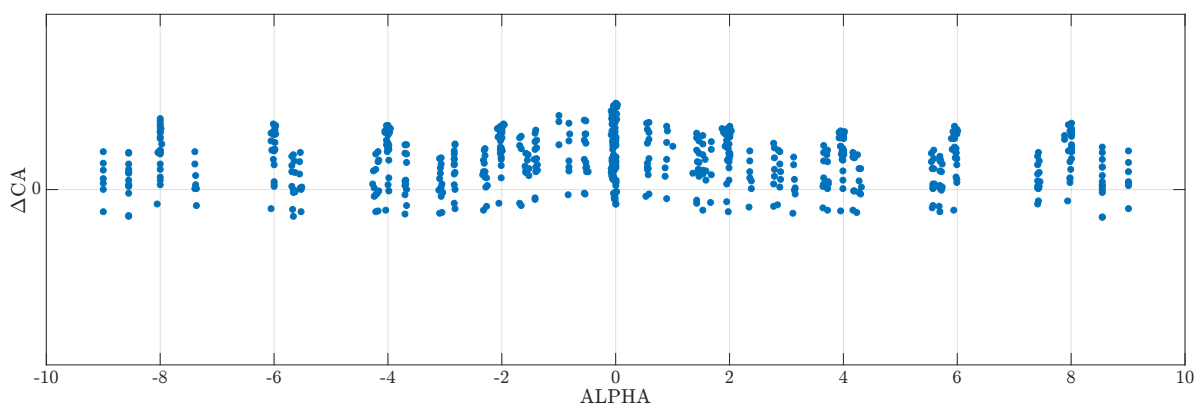

(a) Plotted as a Function of Alpha

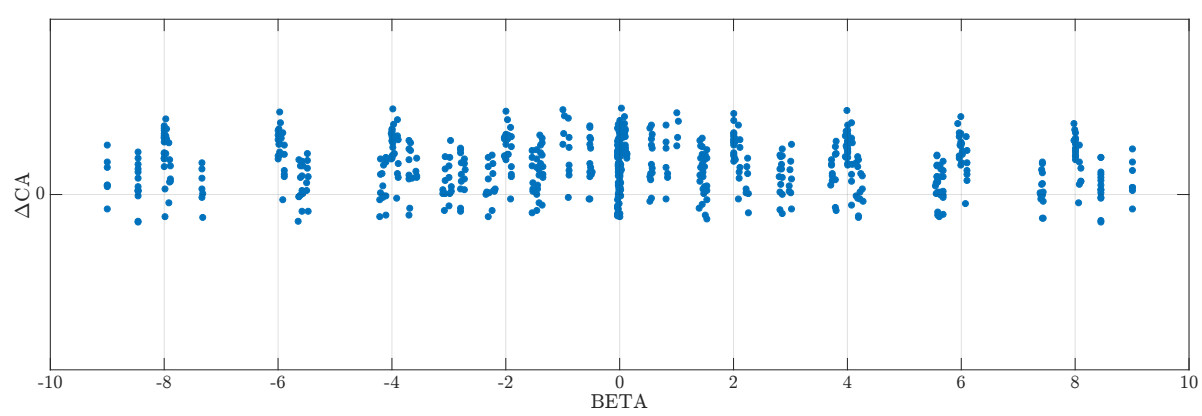

(b) Plotted as a Function of Beta

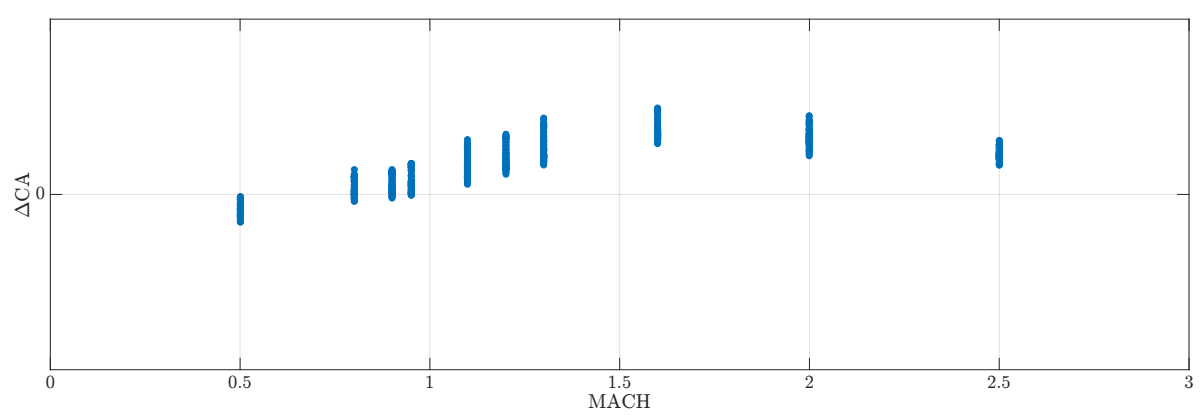

(c) Plotted as a Function of Mach

Fig. 24 Difference of wind tunnel and CFD at wind tunnel conditions based axial force coefficient $(\triangle C A)$ values. 


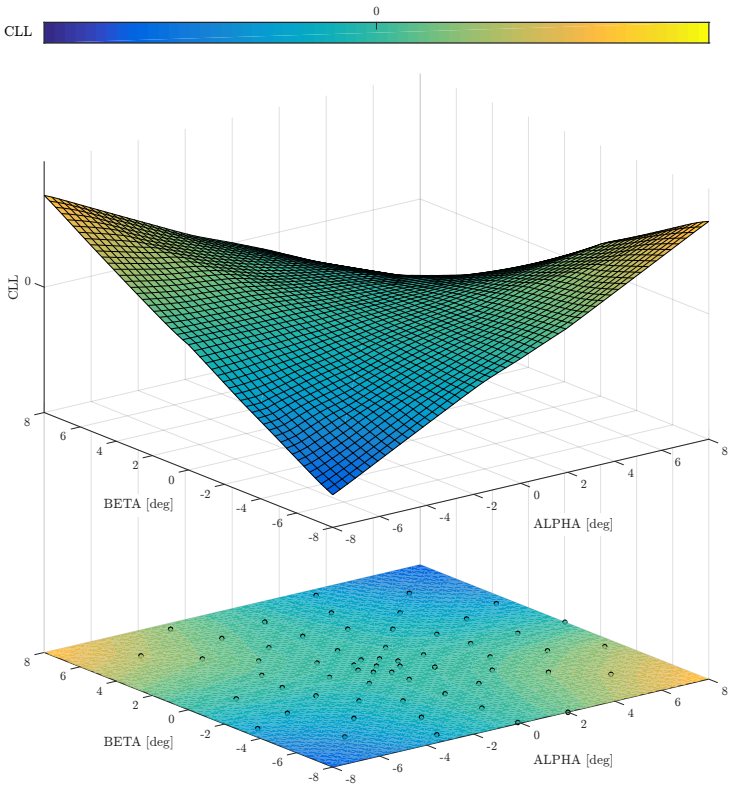

(a) CFD at Wind Tunnel Conditions

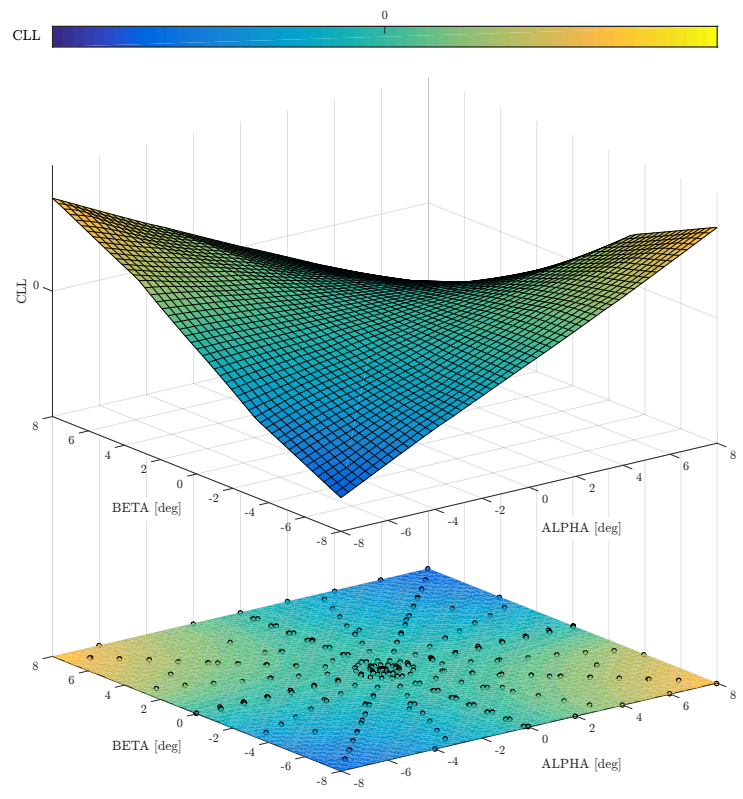

(b) Wind Tunnel Database

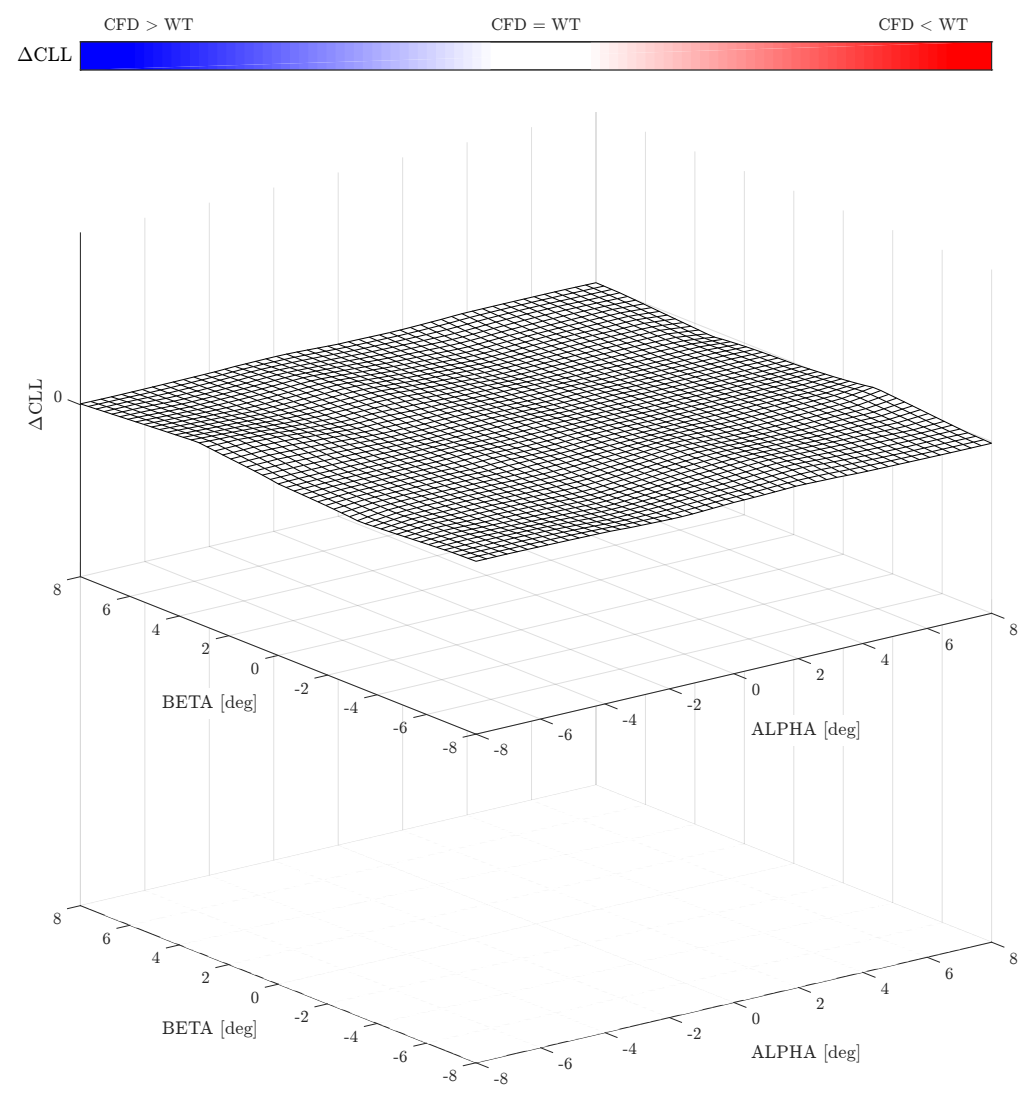

(c) Difference Between Wind Tunnel Database and CFD Solution at Wind Tunnel Conditions

Fig. 25 Comparison of CFD and wind tunnel based rolling moment coefficient ( $C L L$ and $\triangle C L L$ ) response surfaces at Mach 1.20. 


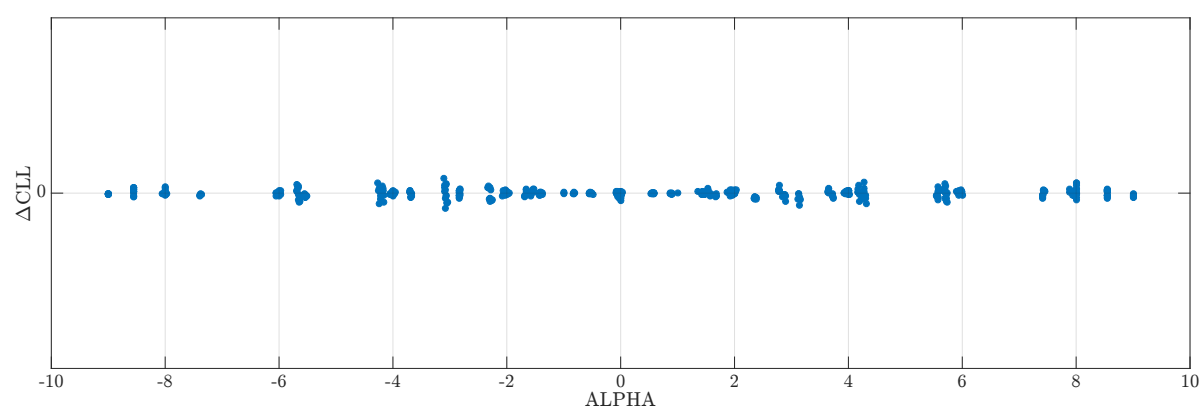

(a) Plotted as a Function of Alpha

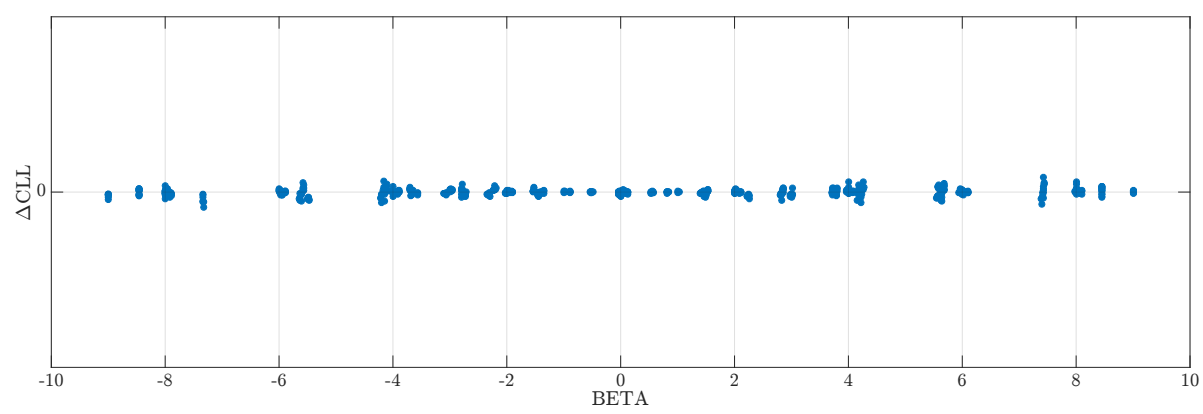

(b) Plotted as a Function of Beta

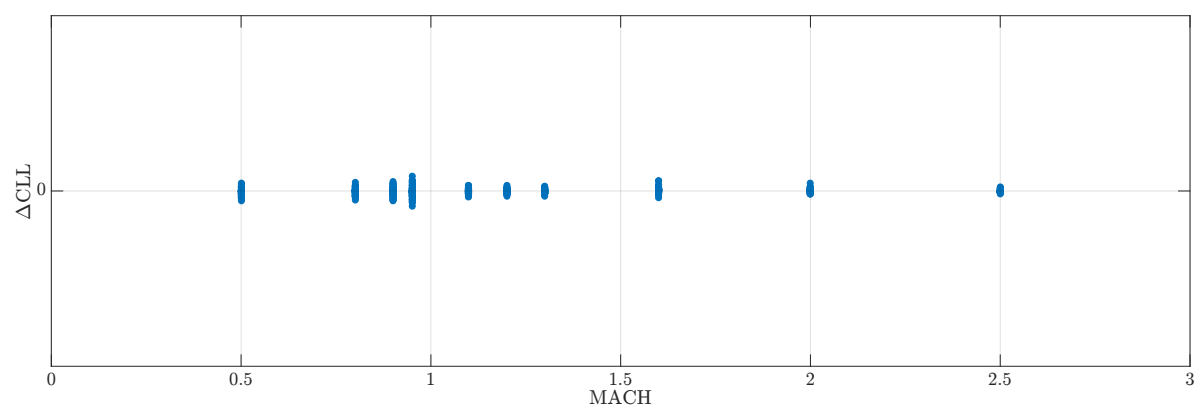

(c) Plotted as a Function of Mach

Fig. 26 Difference of wind tunnel and CFD at wind tunnel conditions based rolling moment coefficient $(\triangle C L L)$ values.

\section{Uncertainty Quantification}

Uncertainty bounds for the ascent database were quantified using a methodology developed for previous launch vehicle force and moment databases constructed from wind tunnel data $[18,19]$. The uncertainty bounds are a function of Mach number and include the contributions of experimental, ground to flight, and modeling uncertainty.

Individual sources of quantifiable error were identified, and separate uncertainty terms were developed for each error source and each aerodynamic coefficient. The individual uncertainty terms were then root-sum-squared to obtain a \pm 3 standard deviation ( $99.7 \%$ confidence) equivalent uncertainty bounds for each aerodynamic coefficient. Since this database was constructed using data from two wind tunnel facilities, separate uncertainties were developed for the Mach ranges corresponding to each facility: Mach $=0.5$ to 1.3 and Mach $=1.6$ to 2.5. All uncertainties associated with wind tunnel data are computed from data measured at the balance moment center. This moment reference location was selected because the correlations between the measured values are minimized due to the balance placement relative to the center of pressure. 
The SLS aerodynamics design team applied range analysis (adapted from Wheeler [20]) to the uncertainty quantification of both repeat data and data sets that theoretically should be similar (such as the difference between a known applied load and the measured value of said load). In this analysis approach, the computed differences between data sets are treated as if the two data sets come from the same normally distributed population with the means of the two groups removed. The differences between each set of points are converted to ranges by taking the absolute value of the differences. The ranges are displayed in a histogram, and a plot of the theoretical distribution of the ranges is superimposed on the histogram. The theoretical range distribution is computed from the estimated population standard deviation of the ranges and assumes the data sets come from the same normally distributed population. The histogram of the actual ranges is compared with the theoretical range distribution to quickly identify any outliers and determine how or if the actual data can be pooled. This approach also gives defensible uncertainty bounds on the data.

\section{A. Experimental Uncertainty}

The experimental uncertainty includes estimates of the force balance measurement error and repeatability of the wind-on aerodynamic data. Measurement error of the facility parameters (pressures, temperatures, Mach number, etc.) are assumed to be small relative to the balance error and repeatability and are not included in the uncertainty quantification. The uncertainty associated with the balance was quantified from a range analysis of the calibration residuals and reflects the balance calibration fit error. The repeatability was quantified using range analysis of the repeat run data acquired during each wind tunnel test.

\section{Balance Accuracy}

The balance calibration fit error $\left(U_{b a l}\right)$ for the MC-12H-1.75A balance used in the Ames UPWT testing was estimated from the calibration load residuals. The test team made a special request to have the load residuals included with the calibration report. The Ames UPWT balance group computed the residuals from voltages to engineering units using BALFIT and provided the results to the team as an output file. The load residuals were then converted to ranges by taking the absolute value of the residuals, and the ranges for each balance axis were plotted in separate histograms as shown in Figure 27. The theoretical normal distribution of ranges is the red curve shown in each range histogram.

The absolute residuals (histogram bars) were generally normally distributed when compared to the theoretical distribution (red curve). The pitching and yawing moment residual distributions had slightly heavy tails, which corresponded to the largest normal and/or side force calibration loads. The calibration fit error, $U_{b a l}$, was defined to cover $99.7 \%$ of the absolute residuals, which is represented by the vertical purple lines shown in Figure 27. Since the calibration residuals were provided in engineering units, the uncertainty bound for each balance component was likewise defined in engineering units. This bound was converted to coefficient form, so that it could be combined with other error terms, using the nominal dynamic pressure at each Mach number and Reynolds number tested, resulting in $U_{b a l}$ that varies with Mach number from Mach=0.5 to 2.5.

\section{Repeatability}

A repeatability analysis of the data acquired in each Ames UPWT test section was performed to estimate the experimental error of the wind tunnel data used to construct the database $\left(U_{r p t}\right)$. Repeat pitch and roll sweeps were obtained in the 11-Foot TWT at all Mach numbers. In the 9- by 7-Foot SWT test, repeat angle of attack sweeps were acquired at all Mach numbers tested. Each run was interpolated to the nominal (scheduled) set point angles, and the range was computed for each set of repeat points. The repeatability was not significantly affected by different model configurations or model attitudes. This allowed for the pooling of the ranges for each aerodynamic coefficient. The bounds for $U_{r p t}$ were defined to cover $99.7 \%$ of the ranges. For each aerodynamic coefficient, there was one value of $U_{r p t}$ for Mach numbers 0.5 to 1.3 (11-Foot TWT), and another value for Mach numbers of 1.6 to 2.5 (9- by 7-FootSWT). Histograms of the ranges computed from repeat runs acquired during the 11-Foot TWT test are presented in Figure 28. The range histograms compare well to the theoretical range normal distributions (red curves), with a small number of outliers occurring in CLM and CLN, such that $99.7 \%$ bounds provides conservatism for these two coefficients. 


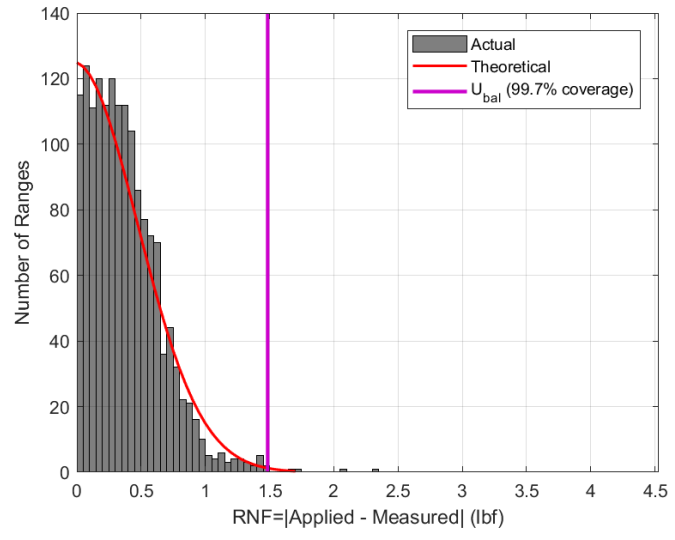

(a) Normal force

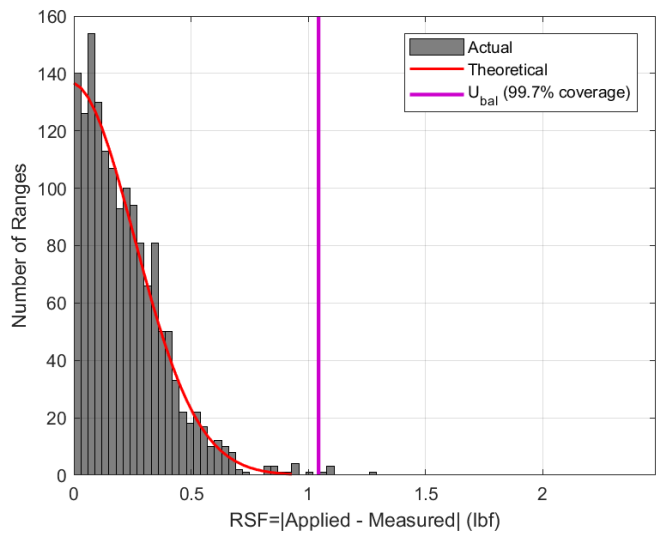

(c) Side force

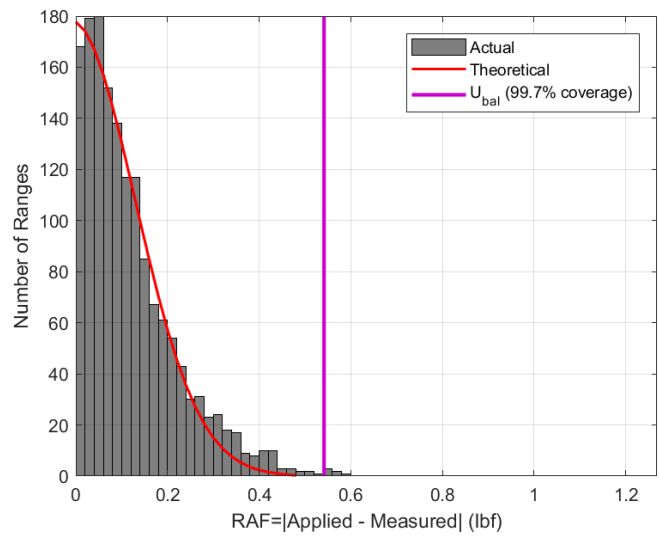

(e) Axial force

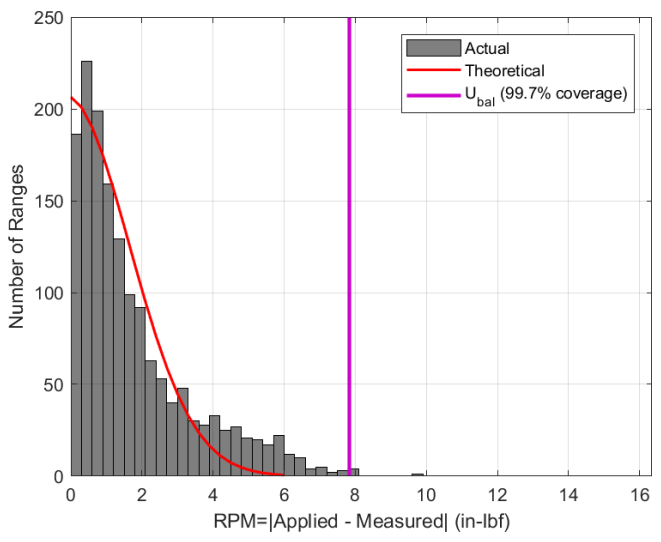

(b) Pitching moment

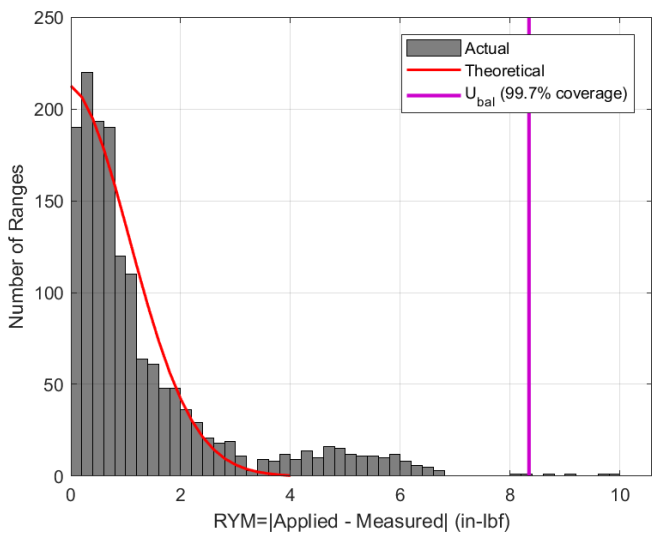

(d) Yawing moment

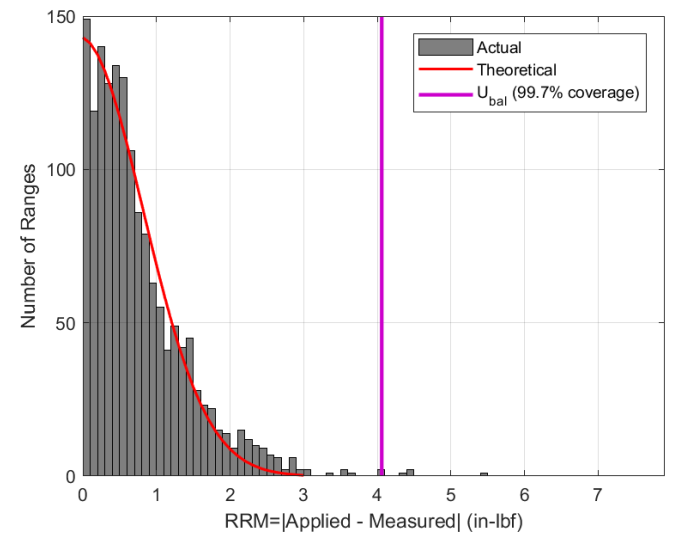

(f) Rolling moment

Fig. 27 Range histogram of MC-12H-1.75A balance calibration residuals. 


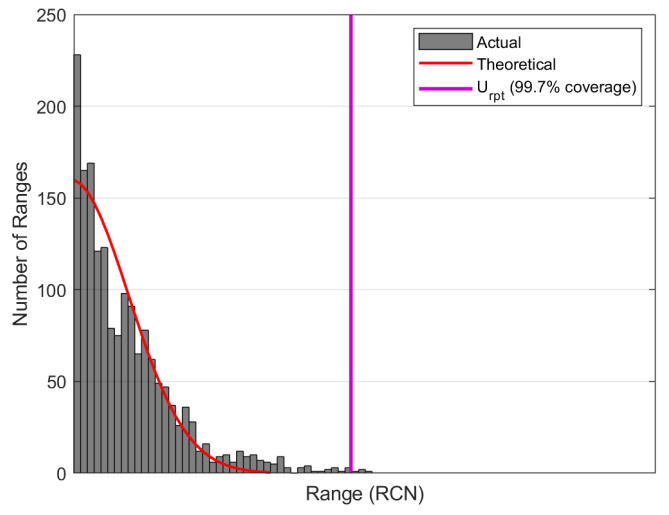

(a) $C N$

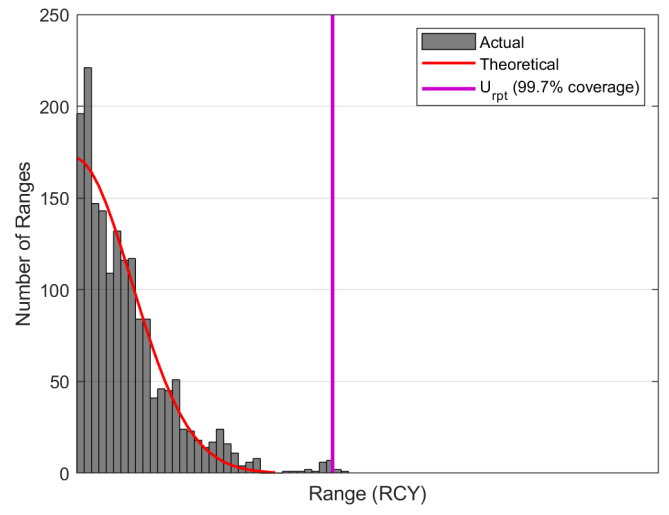

(c) $C Y$

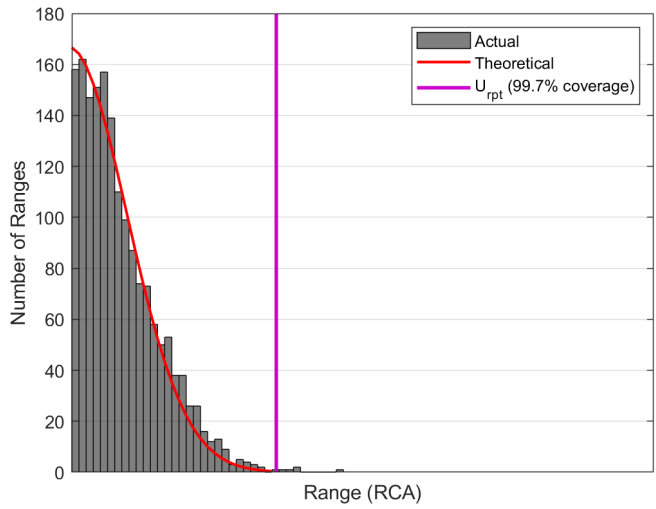

(e) $C A$

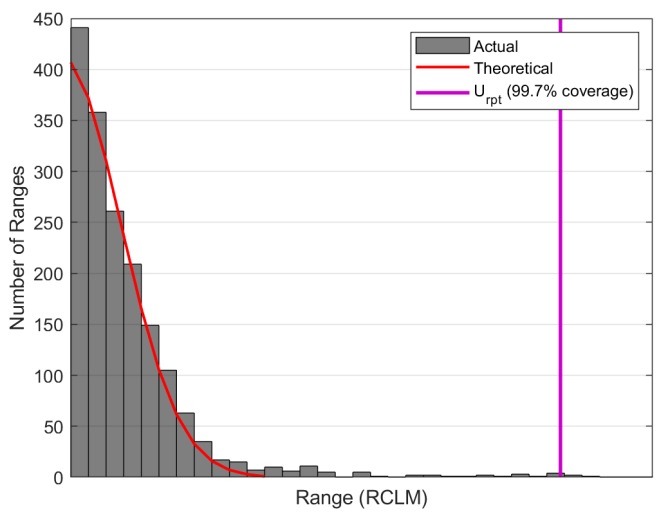

(b) $C L M$

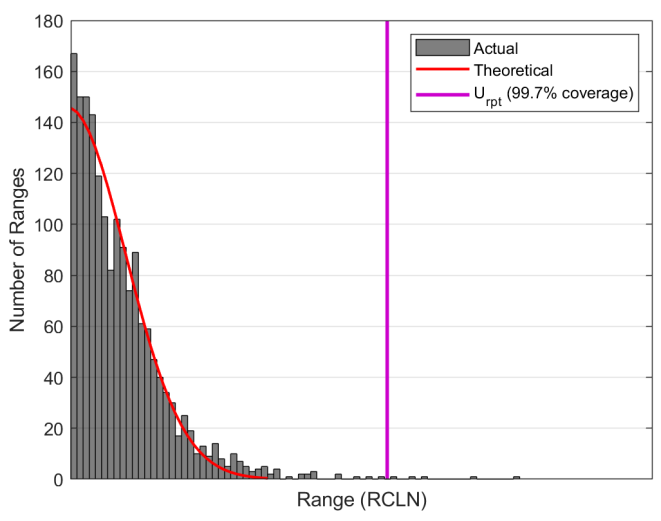

(d) $C L N$

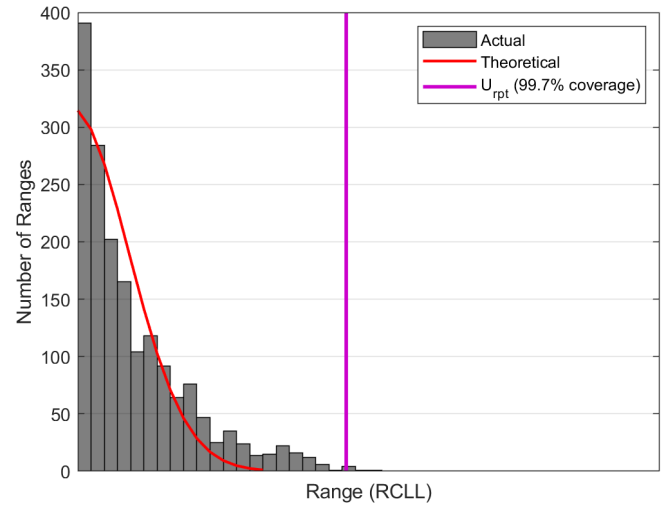

(f) $C L L$

Fig. 28 Range histograms of Ames UPWT 11-Foot TWT test repeatability (Mach=0.5 to 1.3). 
Range histograms from the repeat data acquired during the 9- by 7-Foot SWT test are presented in Figure 29. The repeat data in the 9- by 7-Foot SWT test were obtained in groups of three runs, and the theoretical range normal distribution is different for groups of $n=3$ than for groups of $n=2$, as illustrated in Figure 29. Since the run matrix for this test was smaller, fewer repeat groups were acquired than in the 11-Foot TWT test, which explains the coarser appearance of these histograms. However, the range histograms generally follow the shape of the theoretical range distribution for groups of three. The outliers were inspected and identified as coming from one or two repeat groups at a particular Mach number.

\section{B. Database Modeling Uncertainty}

Two sources of modeling error were quantified for this database. The first term was intended to account for uncertainty due to the experimental data being manipulated to create the final database $\left(U_{D B M}\right)$. This would include activities such as smoothing, fitting, or averaging the source data. To quantify this error, the database values were compared to the wind tunnel data used to construct the database. The second term was a model validation term $\left(U_{t s t}\right)$ that estimated how well the database predicted values of valid test data, which were not used as inputs when generating the database response surfaces.

Figures 30 and 31 show the locations of the wind tunnel data points (black dots) used to construct the database for Mach numbers of 0.5 to 1.3 and 1.6 to 2.5 , respectively. The red dots represent the locations of the database breakpoints. To quantify $U_{D B M}$, the database was queried at every combination of Mach number, angle of attack, and sideslip angle of the wind tunnel data used to construct the database. The modeling residuals were computed as the absolute differences between the measured wind tunnel data and the database values queried at the same points. The value of $U_{D B M}$ was defined to cover $99.7 \%$ of the residuals from each Mach range. Range histograms of the absolute modeling residuals for Mach numbers from 0.5 to 1.3 are presented in Figure 32. Overall, the histograms in Figure 32 compare favorably to the theoretical range distributions, with slightly heavy tails observed in the moment residuals. With the residuals organized in histograms, it is relatively simple to identify the outliers and investigate their causes or trends. In the case of these modeling residuals, the outliers consistently occurred at angles of attack or sideslip near the outer edges of the database. The $99.7 \%$ coverage thus provides a level of conservatism to the database uncertainties. Similar histograms were generated for the modeling residuals computed for Mach numbers of 1.6 to 2.5. Note in Figure 31 that the data acquired at these Mach numbers were obtained at nominally the same angles of attack and sideslip as the database breakpoints. Since there was minimal interpolation or any other manipulations required for these data, the modeling uncertainty for Mach $\geq 1.6$ was smaller than $U_{D B M}$ for Mach numbers from 0.5 to 1.3.

There were several pitch sweeps acquired at fixed yaw angles in the Ames UPWT 11-Foot TWT that were unused as part of the database construction. These data were used as "test" points to compare with the database response surfaces that were constructed from data obtained at different model angles. These comparisons were the basis for the development of the model validation term, designated as $U_{t s t}$. The database was queried at these "unused" wind tunnel data set points (black circles in Figure 33), and the absolute difference between the queried value and the actual wind tunnel data point was computed as a residual. The bound for $U_{t s t}$ was set to cover $99.7 \%$ of these residuals.

There were no such "unused" runs acquired in the 9- by 7-Foot SWT, so a smaller subset of the residuals was used as a surrogate for Mach numbers from 1.6 to 2.5. The data from the 9- by 7-Foot SWT were acquired at evenly spaced angles of attack and sideslip at or very close to the database breakpoints (see Figure 31). Therefore, to estimate $U_{t s t}$ for Mach=1.6 to 2.5, the "unused" residuals (black circles in Figure 33) that are located farthest from the points used to build the database (red dots in Figure 33) were excluded from computing the 99.7\% coverage. In other words, the residuals from "unused" data at nominal values of $(\alpha, \beta)=\left\{\left( \pm 2^{\circ}, \pm 8^{\circ}\right),\left( \pm 6^{\circ}, \pm 4^{\circ}\right),\left( \pm 6^{\circ}, \pm 8^{\circ}\right)\right\}$ were omitted and the value of $U_{t s t}$ for Mach=1.6 to 2.5 was defined to cover $99.7 \%$ of the remaining residuals. 


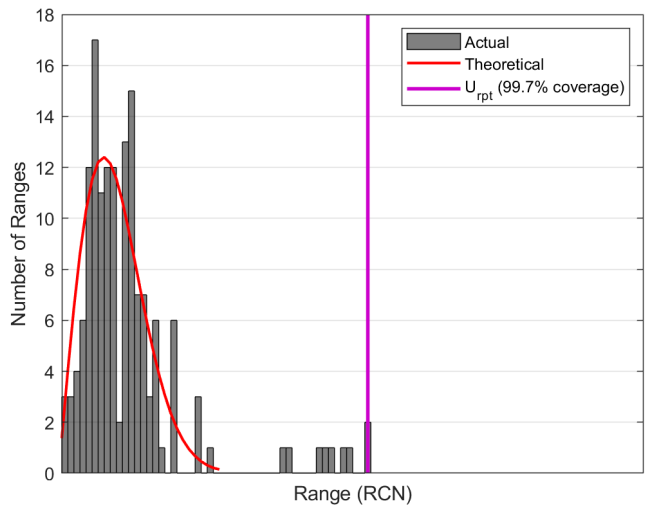

(a) $C N$

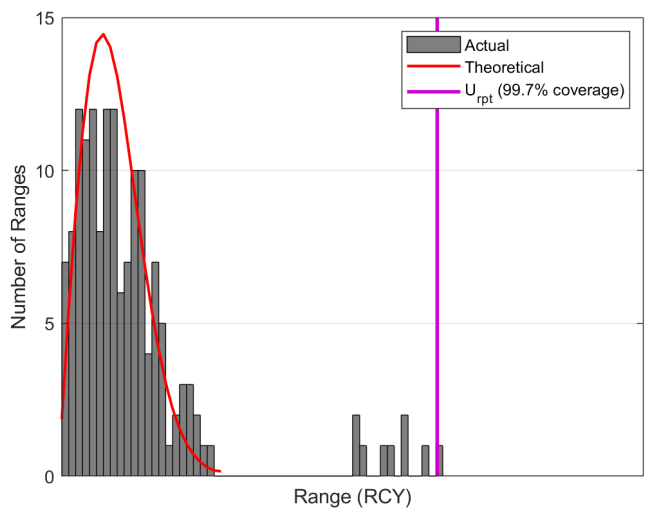

(c) $C Y$

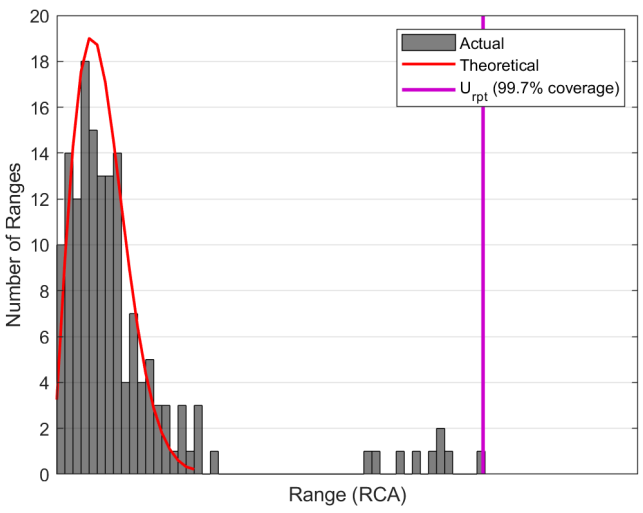

(e) $C A$

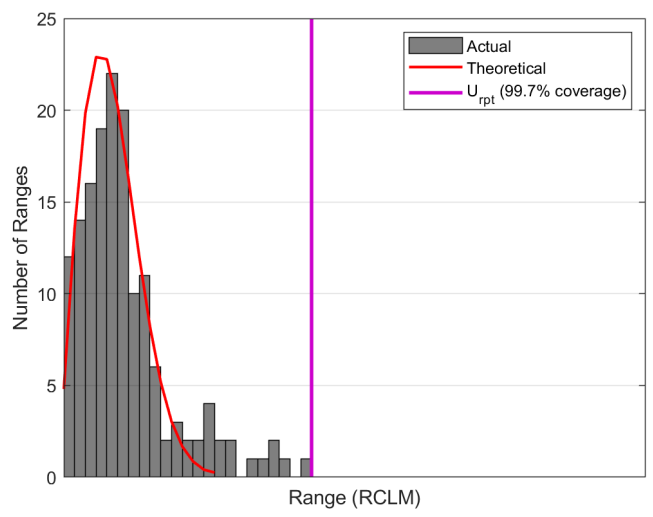

(b) $C L M$

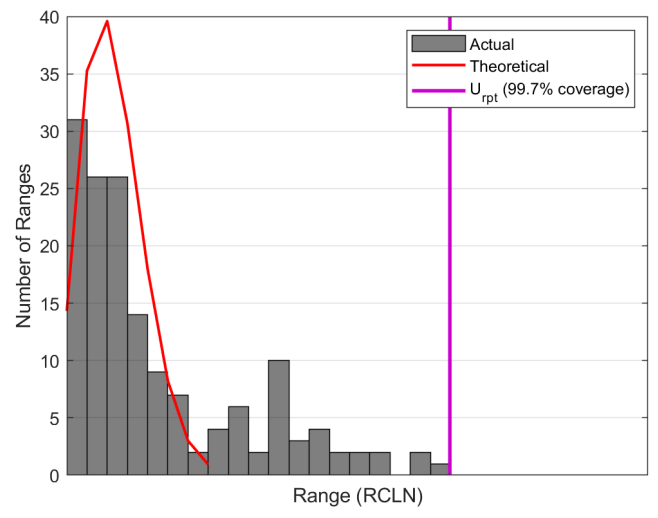

(d) $C L N$

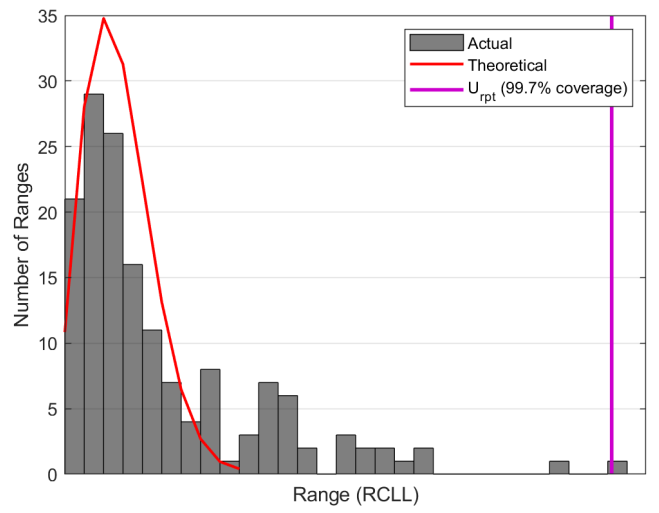

(f) $C L L$

Fig. 29 Range histograms (n=3) of Ames UPWT 9- by 7-foot test repeatability (Mach=1.6 to 2.5). 
- Measurements - Database

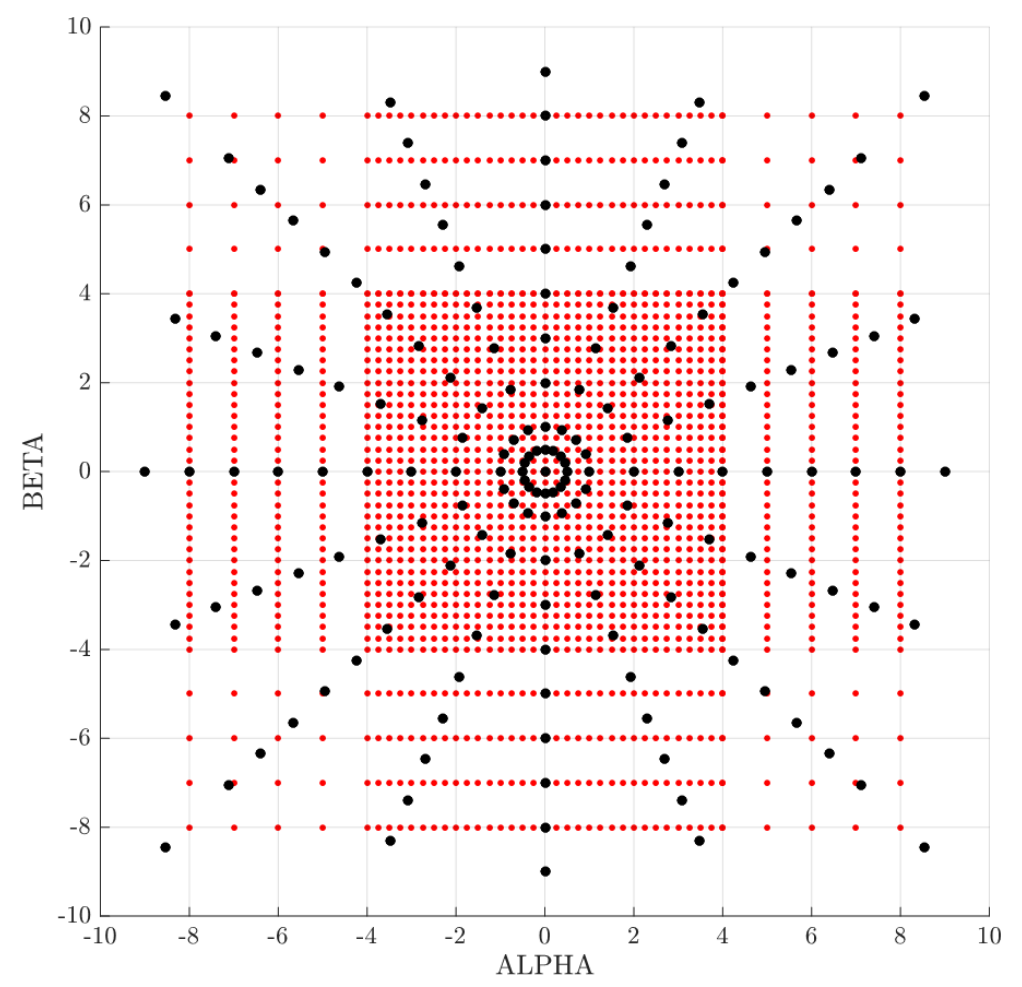

Fig. 30 Comparison of measured data points from the Ames UPWT 11-Foot TWT test (black) and database breakpoints (red).

- Measurements

- Database

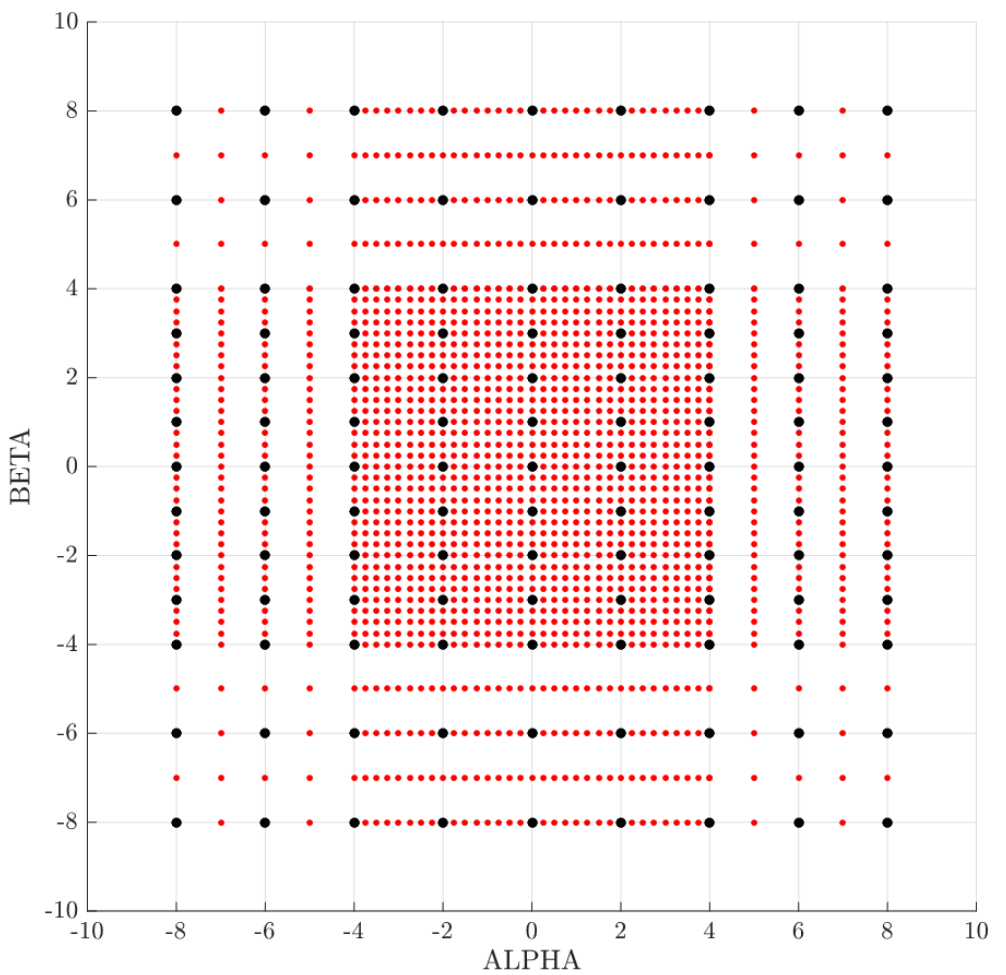

Fig. 31 Comparison of measured data points from the Ames UPWT 9- by 7-Foot SWT test (black) and database breakpoints (red). 


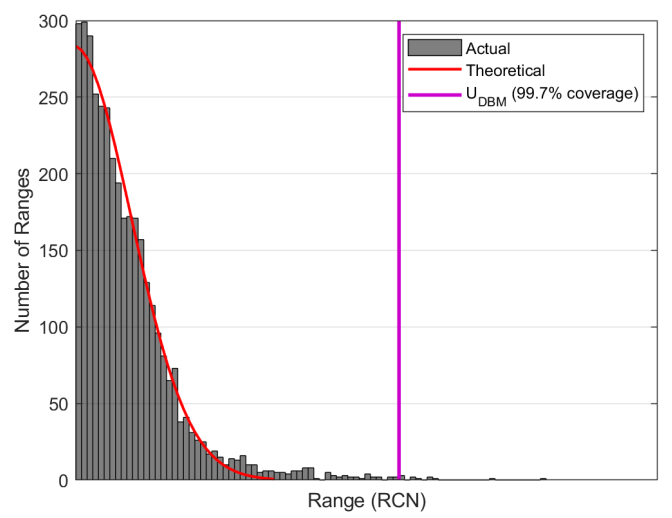

(a) $\mathrm{CN}$

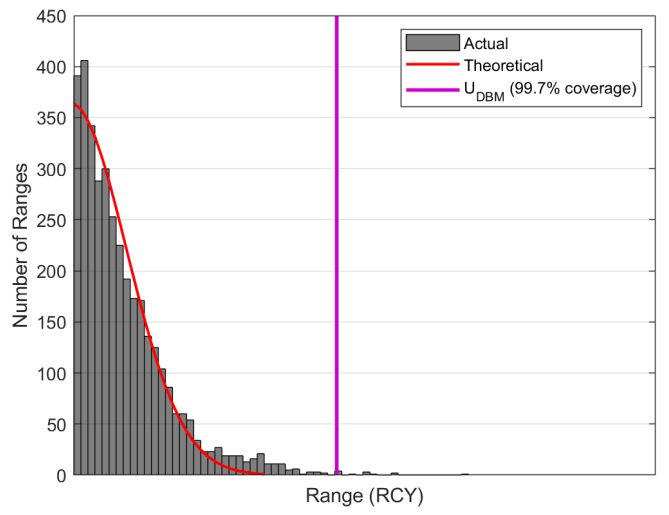

(c) $C Y$

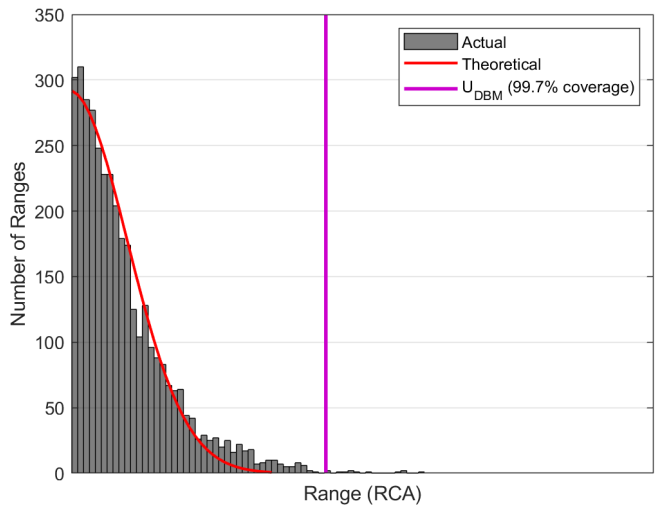

(e) $C A$

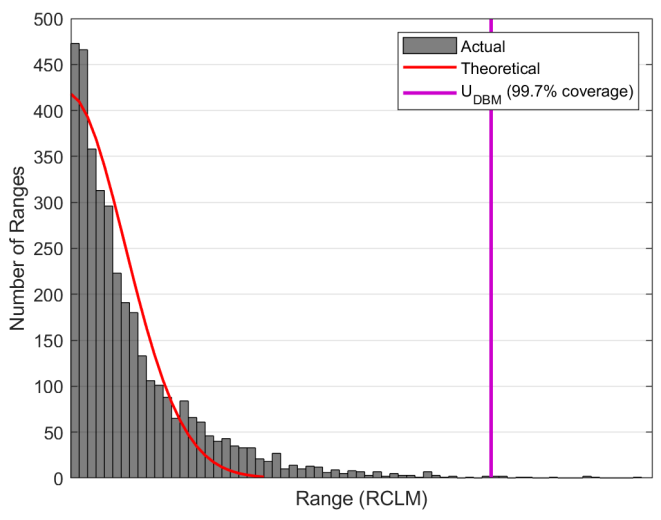

(b) $C L M$

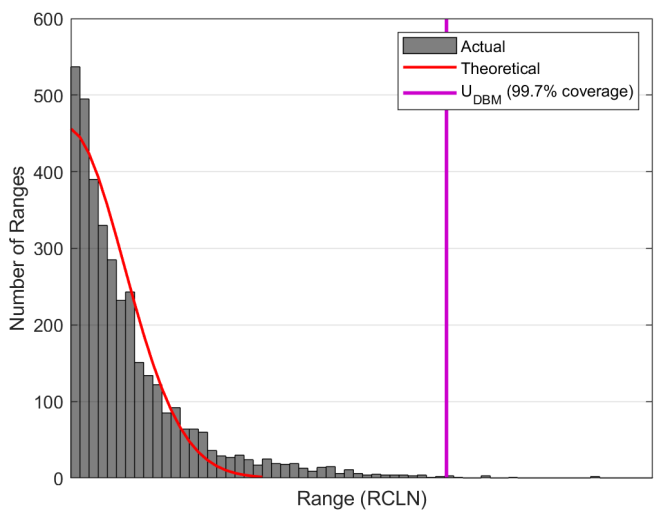

(d) $C L N$

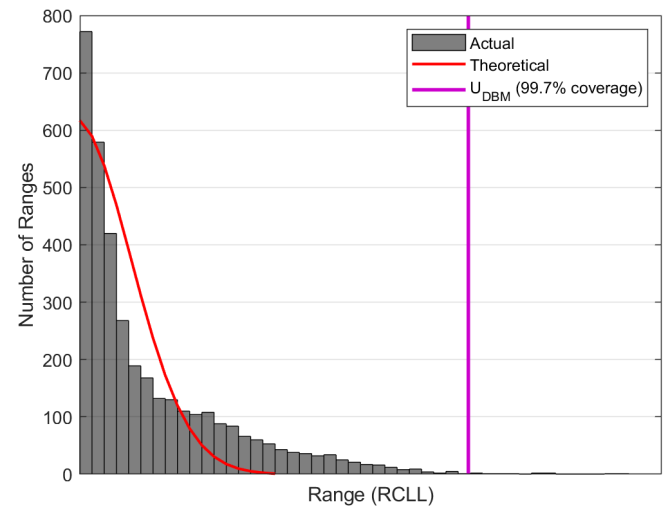

(f) $C L L$

Fig. 32 Range histograms of absolute differences between wind tunnel data and database values at wind tunnel data conditions, for Mach $=0.5$ to 1.3 . 


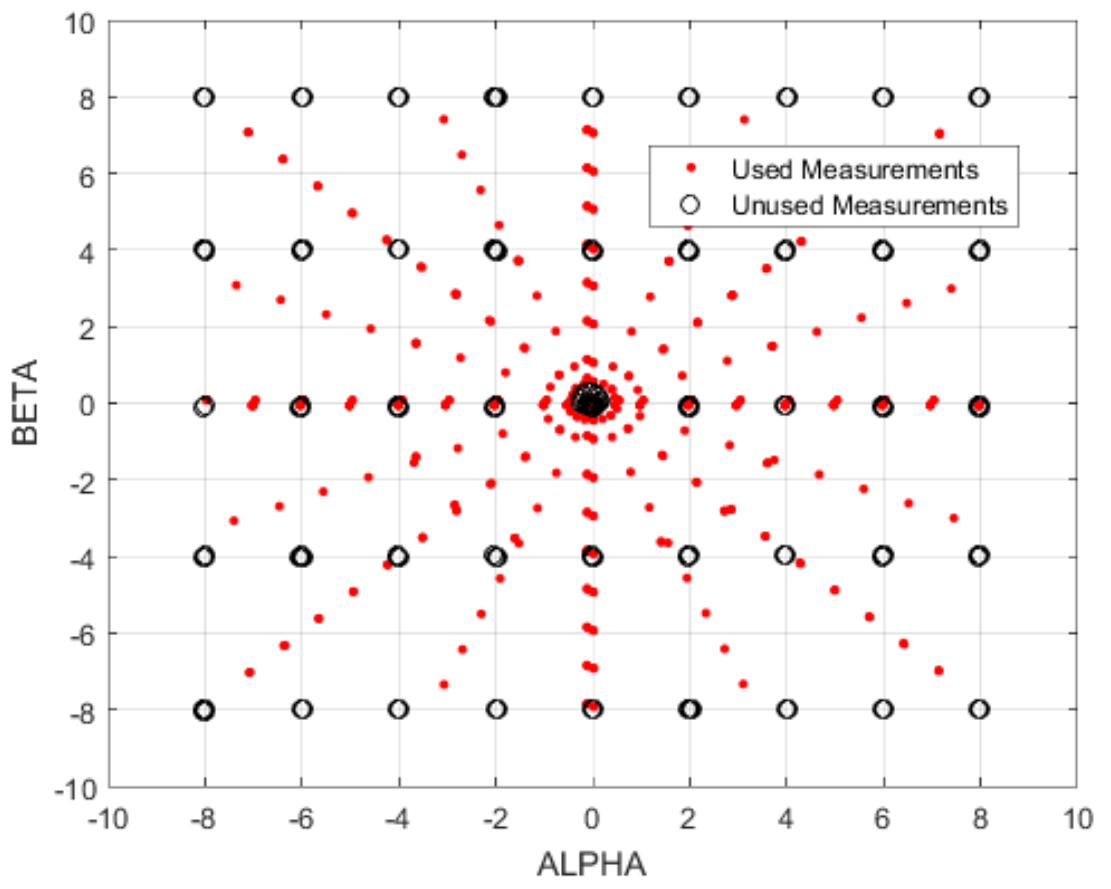

Fig. 33 Comparison of measured data points from the Ames UPWT 11-Foot TWT that were either used (red) or unused (black) during database construction.

Since the database modeling and validation error terms are based on residuals computed from wind tunnel data, it is important to remove the contribution of repeatability error in these modeling error terms. Therefore, $U_{r p t}$ was subtracted from the database modeling and validation uncertainty terms in order to not double count the experimental error in the total uncertainties. If $U_{r p t}>U_{D B M}$, then $U_{D B M, n e t}$ simply becomes zero. Likewise, if $U_{r p t}>U_{t s t}$, then $U_{\text {tst, net }}$ is zero.

$$
\begin{aligned}
U_{D B M, n e t} & =\sqrt{U_{D B M}^{2}-U_{r p t}^{2}} \\
U_{t s t, n e t} & =\sqrt{U_{t s t}^{2}-U_{r p t}^{2}}
\end{aligned}
$$

\section{Ground to Flight Uncertainty}

\section{As Delivered}

The ground test to flight uncertainty $\left(U_{G 2 F}\right)$ was developed from CFD data and is intended to cover uncertainties in the database related to:

- sting and wall interference in the wind tunnel

- OML differences between the wind tunnel and flight OMLs

- Reynolds number effects

- plume effects from the core engines and SRBs

However, at the time this database was constructed, there were no CFD solutions completed for the SLS-28k vehicle at flight conditions. Thus, CFD simulations of the SLS-10k configuration were used as a surrogate to develop $U_{G 2 F}$. CFD simulations using USM3D were run at both wind tunnel and flight conditions on the SLS-10003 geometry at Mach numbers ranging from 0.5 to 4.0. There were 107 combinations of Mach number, angle of attack, and sideslip angle for which USM3D simulations were run at both wind tunnel and flight conditions. The difference between each pair of flight and wind tunnel CFD solutions was computed for each aerodynamic coefficient.

These residuals were independent of angle of attack and sideslip and were scattered about zero for every coefficient except $C A$. There was a consistent offset between the flight and wind tunnel CFD predictions of $C A$ that was a function 
of Mach number. The offset itself was not incorporated into the SLS-28k database because these CFD simulations were obtained on an SLS-10003 OML. Thus, an estimate of $U_{G 2 F}$ for $C A$ was obtained by computing the scatter about the mean difference in $C A$ at each Mach number and setting the bound to cover $99.7 \%$ of that scatter. With the exception of $C Y$, where the residuals were pooled across all Mach numbers, $U_{G 2 F}$ bounds were obtained by pooling the ground to flight residuals into two Mach number ranges (Mach = 0.5 to 1.6 and Mach > 1.6) and setting the bounds to cover $99.7 \%$ of the absolute residuals in each Mach group. Plots of the CFD residuals and corresponding $U_{G 2 F}$ bounds are presented in Figures 34 - 36. In general, the difference between the CFD results at flight and wind tunnel conditions was largest for Mach numbers from 0.5 to 1.6. It is important to note that since the CFD pitching and yawing moment coefficients were computed about a nominal center of gravity location (rather than the experimental balance moment center), the residuals and subsequent ground to flight uncertainty bounds for CLM and CLN are determined with respect to this reference location, and then later transferred to the wind tunnel data balance moment center before combining with the other error terms to compute the total uncertainty.

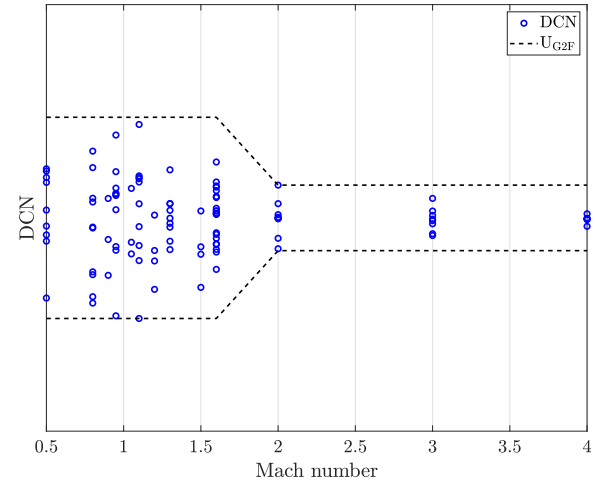

(a) $C N$

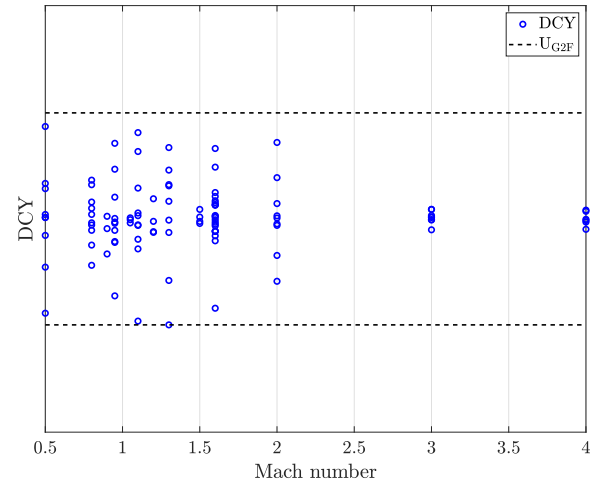

(b) $C Y$

Fig. 34 Differences between flight and wind tunnel CFD for $C N$ and $C Y$.

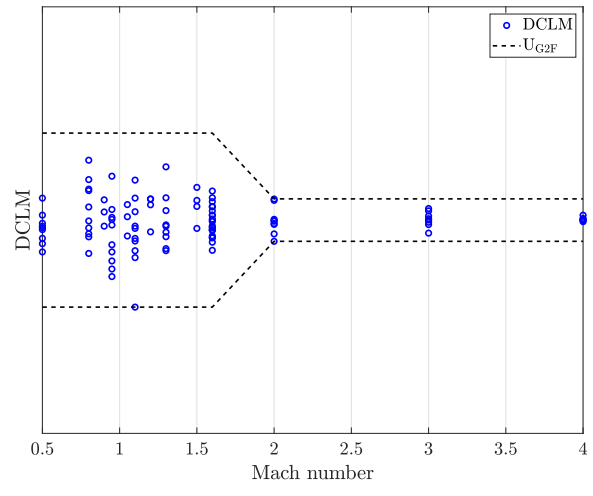

(a) $C L M$

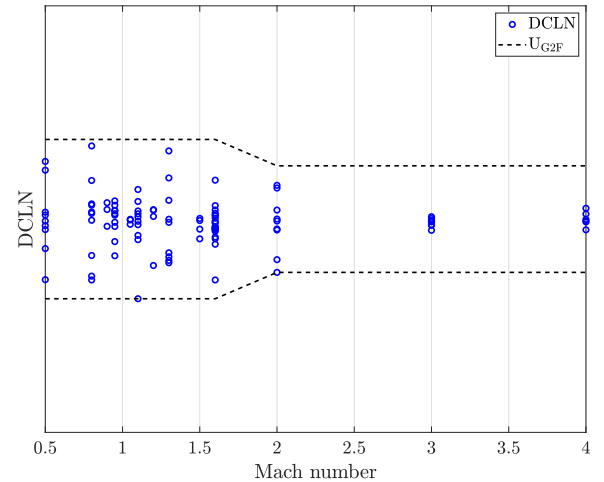

(b) $C L N$

Fig. 35 Differences between flight and wind tunnel CFD for $C L M$ and $C L N$ computed at a nominal center of gravity location. 


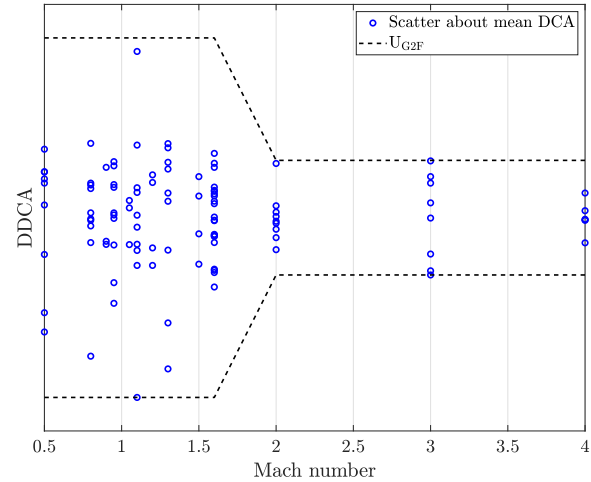

(a) Scatter about mean $D C A$

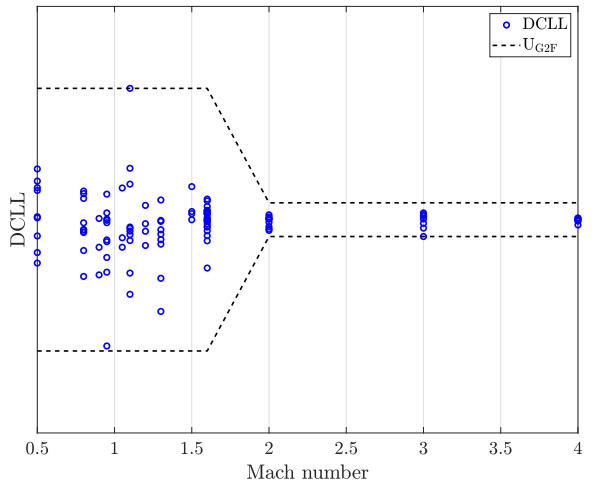

(b) $C L L$

Fig. 36 Differences between flight and wind tunnel CFD for $C A$ and $C L L$.

\section{FUN3D Development}

For future revisions of the database, the ground to flight uncertainties from USM3D simulating the Block 1 Crew (as discussed in Section VII.C) will be replaced with the FUN3D simulations of the Block 1B Crew discussed in Section IV. The figures that follow show examples of how the ground to flight uncertainty would be computed using CFD solutions alone. For these comparisons, response surfaces were developed for the FUN3D at flight conditions following the same methods discussed in Section V.B, and direct subtractions between the FUN3D at wind tunnel conditions and flight conditions were computed similar to Figures 15-26. In Figures 37-39, the ordinate scales have been set to match the $\Delta$ scales from Figures 15-26. Comparing the magnitude of the scatter for the wind tunnel to CFD at wind tunnel conditions differences to CFD at wind tunnel and flight conditions differences, it can be seen that the scatter between the two CFD solutions is higher for $\triangle C N$ across the entire Mach range, and higher for $\triangle C Y$ and $\triangle C L N$ at high Mach numbers. $\triangle C L M$ between the two CFD solutions does not show strong Mach dependence in contrast to the wind tunnel to CFD comparison that showed a peak in the scatter at Mach 0.95. $\triangle C A$ shows a trend as a function of Mach number, but this trend is different than seen between the wind tunnel and CFD solutions (Fig. 24(c). How these trends in axial force would be accounted for in the database delivery is still to be decided. The scatter seen in $\triangle C L L$ is small compared to the other moments consistent with the wind tunnel to CFD comparisons.

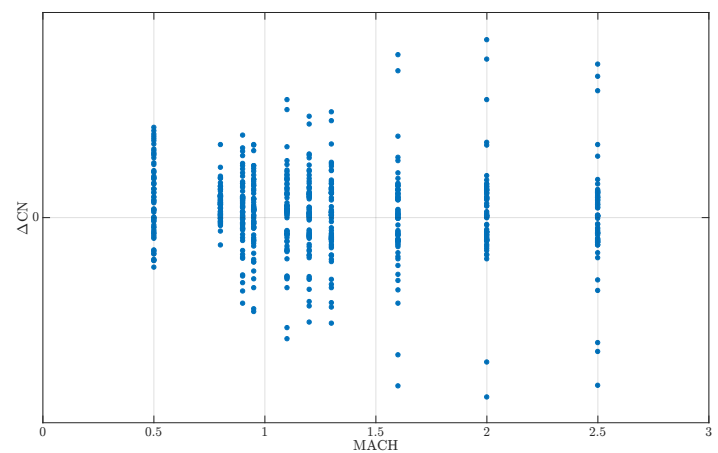

(a) $\mathrm{CN}$

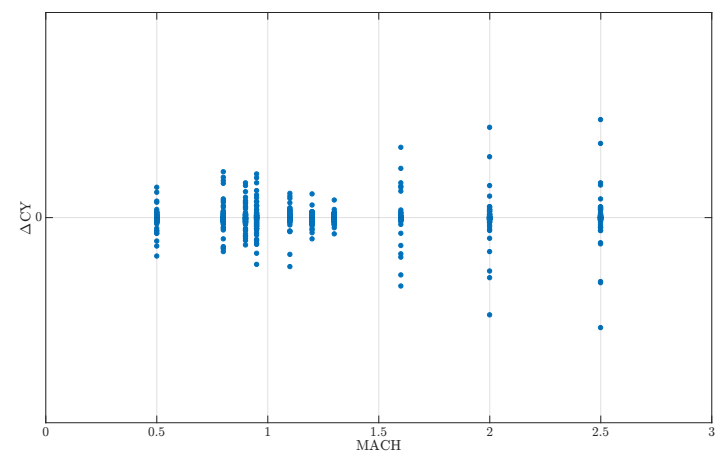

(b) $C Y$

Fig. 37 Differences between FUN3D at wind tunnel and flight conditions for $C N$ and $C Y$. 


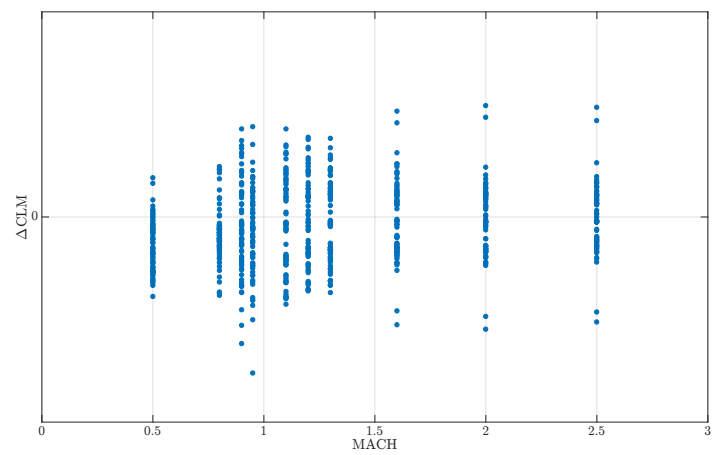

(a) $C L M$

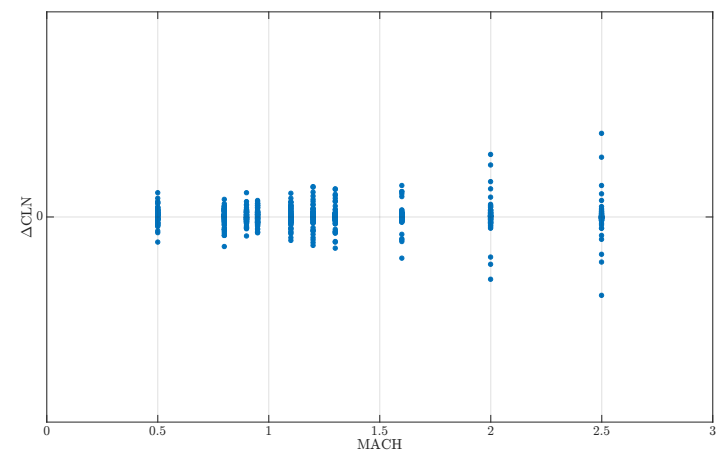

(b) $C L N$

Fig. 38 Differences between FUN3D at wind tunnel and flight conditions for $C L M$ and $C L N$.

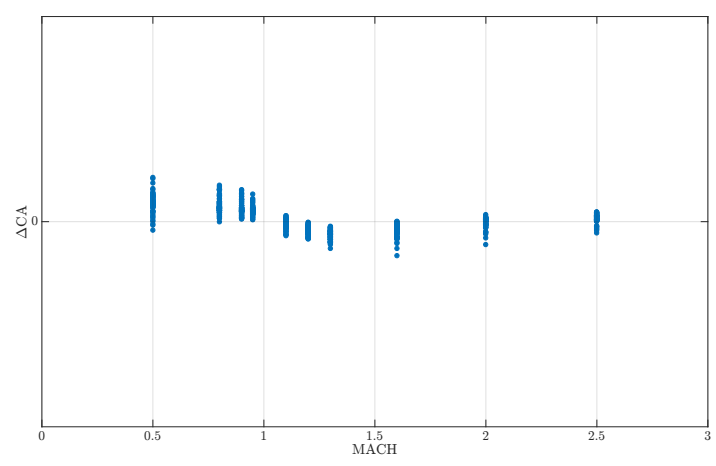

(a) $C A$

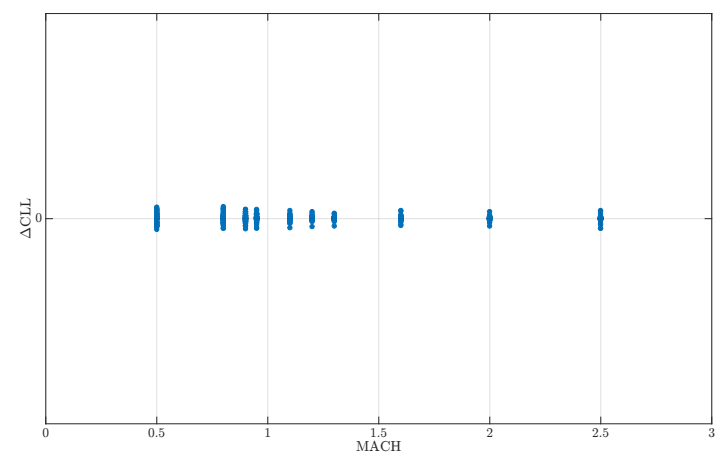

(b) $C L L$

Fig. 39 Differences between FUN3D at wind tunnel and flight conditions for $C A$ and $C L L$.

\section{Final Uncertainty}

The final database uncertainty bound for each coefficient was obtained by root-sum-squaring the individual uncertainty terms together and then applying an uncertainty factor $(U F)$ of 1.05 . The uncertainty factor is intended to account for unknown unknowns and effectively acts as an additive to the quantified uncertainty. The equation for the final uncertainty bounds for the full stack ascent database for Mach 0.5 to 2.5 is given below.

$$
U_{\text {Final }}=U F \sqrt{U_{D B M, n e t}^{2}+U_{t s t, n e t}^{2}+U_{r p t}^{2}+U_{b a l}^{2}+U_{G 2 F}^{2}+U_{W T c o r r}^{2}}
$$

The last term under the square root, $U_{W T c o r r}$, is intended to account for uncertainty due to corrections (such as flow angularity and roll dependent bias) that were applied to the raw balance data. The data used as a reference for these corrections were measured by the balance, and the resulting corrections were based on the addition/subtraction of one set of balance data from another. Therefore, $U_{W T c o r r}$ was defined to be equal to the balance calibration uncertainty $\left(U_{\text {WTcorr }}=U_{\text {bal }}\right)$.

The final uncertainty bounds as computed above are plotted versus Mach number, along with the individual sources of uncertainty, in Figures 40 - 45. For all of the figures here, the values at Mach 1.20 are approximately 5\% of the scales used (vertical axis limits and color map bounds) for the response surface plots in Figures 15, 17, 19, 21, 23, and 25 with the exception of $C L M$ which is closer to $25 \%$. The ground to flight uncertainty is the largest contributor to the total uncertainty at Mach numbers from 0.5 to 1.6, for every coefficient except yawing moment (Figure 43). For all three force coefficients, the contribution of modeling error (solid blue lines) to the final bounds was zero 
because the repeatability uncertainty $U_{r p t}$ was larger than $U_{D B M}$. The model validation uncertainty, $U_{t s t}$, was larger for Mach numbers below 1.6. This was expected because more manipulation and interpolation of the wind tunnel data was required at these Mach numbers, where the data were acquired using pitch and roll sweeps. Since the database nominals were defined at evenly spaced angles of attack and sideslip, the high Mach number data were nominally acquired at database breakpoint values, where the data were acquired using angle of attack and sideslip sweeps in the 9- by 7-Foot SWT (Mach = 1.6 to 2.5 ).

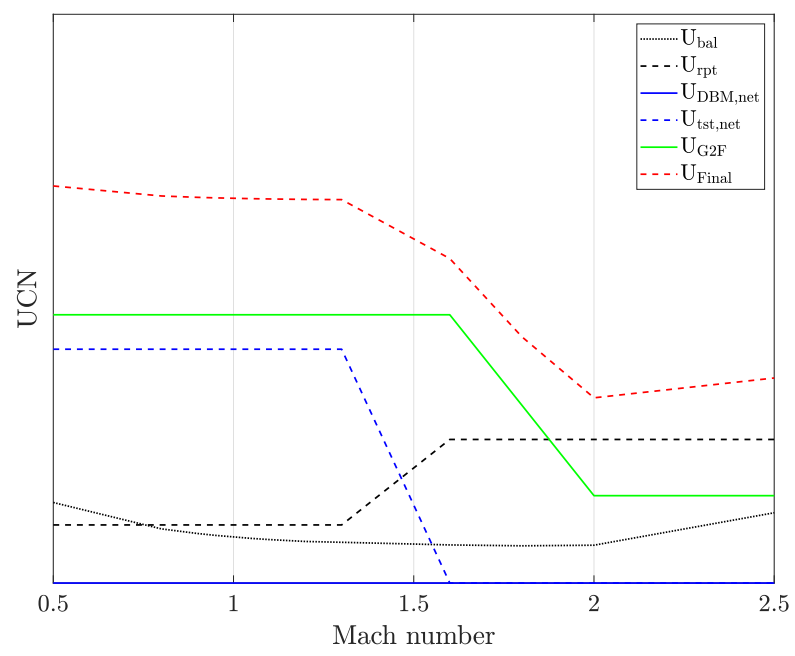

Fig. 40 Database uncertainty build up for $C N$.

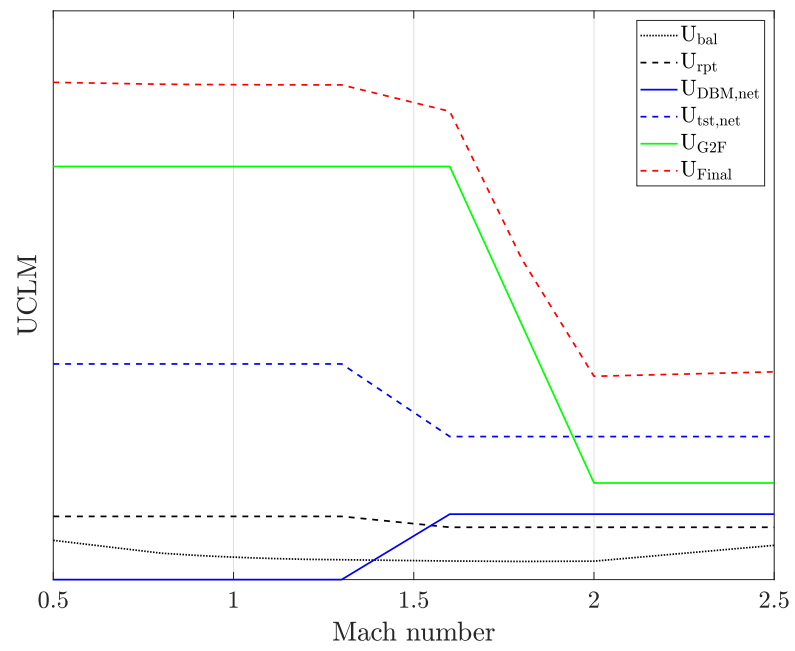

Fig. 41 Database uncertainty build up for $C L M$ at the balance moment center. 


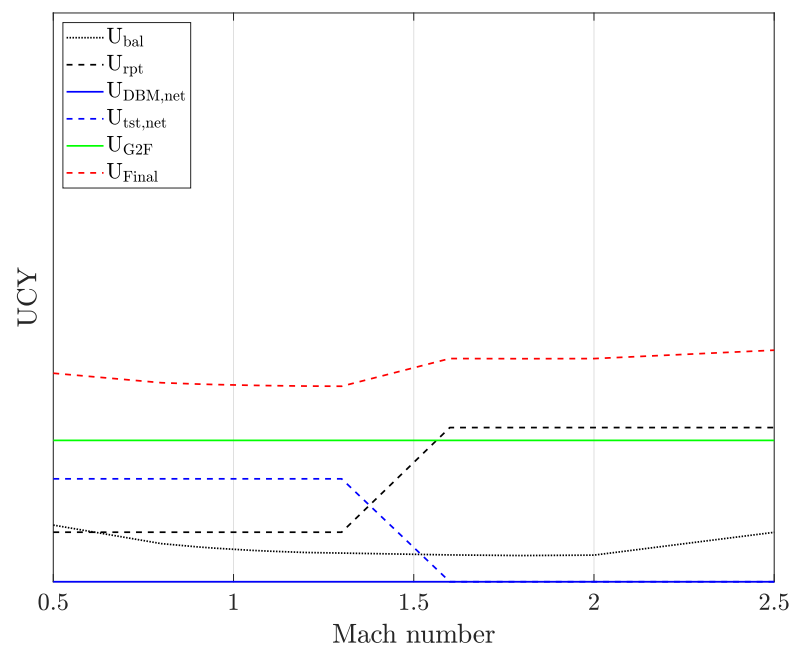

Fig. 42 Database uncertainty build up for $C Y$.

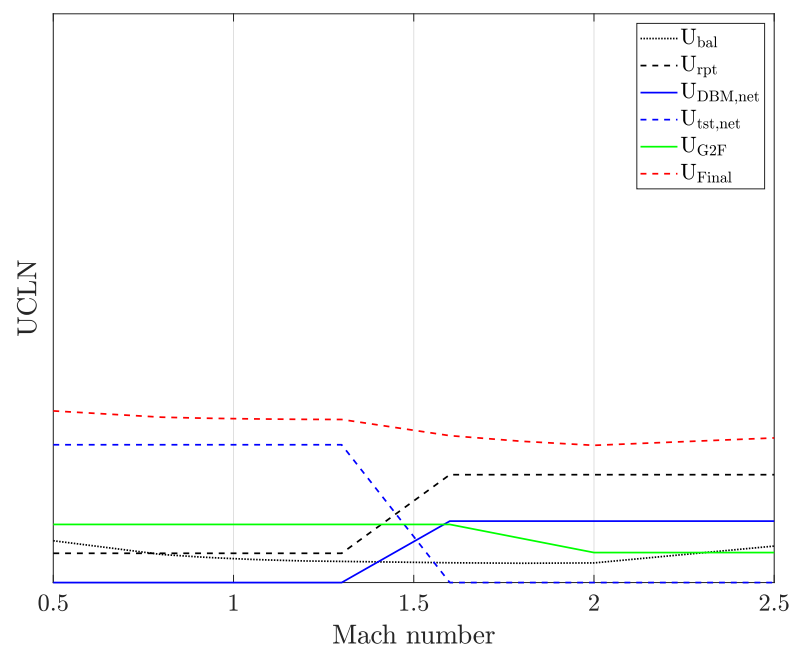

Fig. 43 Database uncertainty build up for $C L N$ at the balance moment center. 


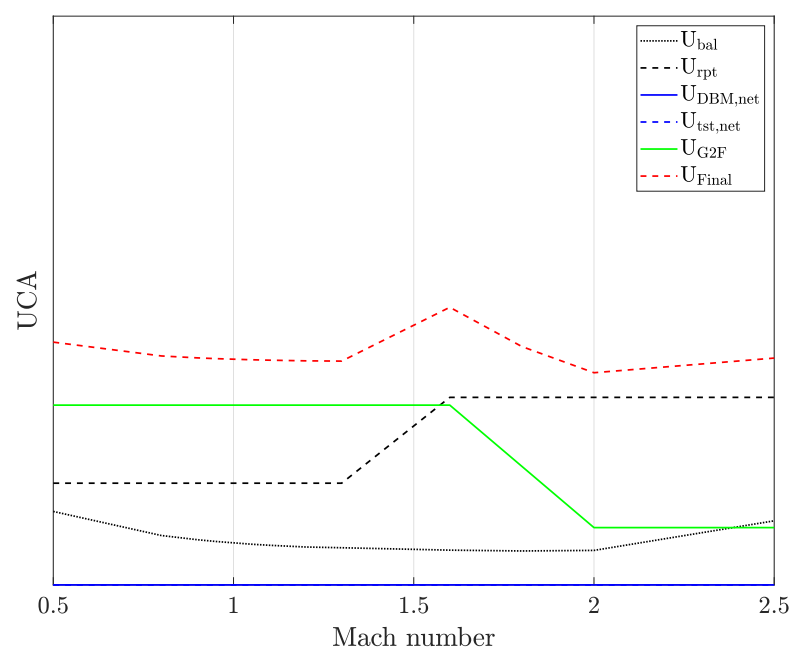

Fig. 44 Database uncertainty build up for $C A$.

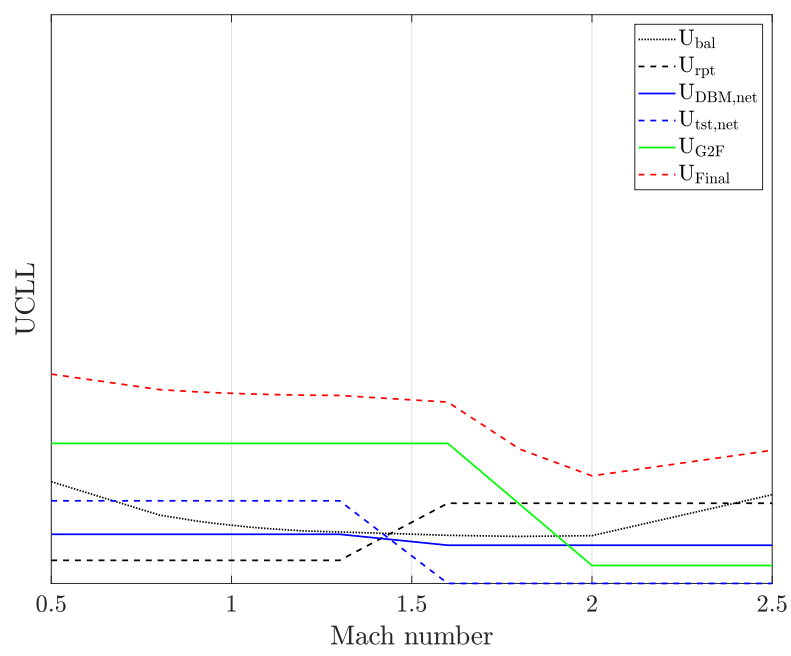

Fig. 45 Database uncertainty build up for $C L L$.

\section{Conclusions}

An ascent force and moment database for the SLS Block 1B Cargo vehicle was developed using a combination of wind tunnel testing and CFD simulations. The wind tunnel tests were performed at the NASA Ames Unitary Plan Wind Tunnel from Mach 0.5 to 2.5. CFD simulations were performed using FUN3D at both wind tunnel test conditions (for verification) and at predicted flight trajectory conditions (for use in other SLS databases). For the nominal force and moment database, the wind tunnel data were used to develop response surfaces as a function of Mach, pitch angle, and sideslip angle. The data reduction included standard corrections from the wind tunnel (flow angularity, buoyancy, etc.) as well as test specific corrections to account for roll dependent bias seen as part of testing on a roll mechanism.

CFD solutions were developed in FUN3D for verification purposes and for use in a number of SLS databases. 
These data were evaluated against the wind tunnel data as part of the verification work. In general, the CFD solutions computed at wind tunnel conditions compared well with the wind tunnel results. The largest differences between the two databases were seen in pitching moment where there was a well defined trend as a function of pitch angle most likely resulting from a difference in the center of pressure. Notable differences were also seen in axial force, but these variations were typically a function of Mach number with the CFD generally predicting a lower value than the wind tunnel test. These data will continue to be assessed with the goal of improving the experimental and CFD best practices.

A detailed uncertainty analysis was performed for delivery with the force and moment database. The total uncertainty was computed using range analysis to assess a number of factors including the experimental uncertainties, database modeling uncertainties, and ground to flight uncertainties developed from CFD simulations. For the current delivery of the database, USM3D simulations on the Block 1 Crew vehicle were used as a surrogate model, but the FUN3D simulations for the Block 1B Crew vehicle will be used for future database deliveries. Previously, the CFD solutions were compared directly to the wind tunnel data, but comparisons between CFD simulations at wind tunnel and CFD simulations at flight conditions will most likely be used moving forward.

\section{Acknowledgments}

The authors would like to thank the SLS program and the past and present SLS ATT leadership (John Blevins, Ricky Campbell, and Andy Herron) for their support of this work. The authors would also like to thank the members of the SLS ATT that helped to support the wind tunnel testing (Dave Purinton, Carlos Baretto, Bill Crosby, Gary Erickson, Ray Gomez, Melody Mayle, and Naomi McMillin), the model designer (Don Morr), the Ames UPWT Test Manager (Steve Buchholz), and the entire Ames UPWT test team who made the wind tunnel test possible. The authors would also like to thank Jim Ross for his contributions to the UPWT test effort.

\section{References}

[1] “Space Launch System,” https://www.nasa.gov/sls, 2018. Accessed: 31 OCT 2018.

[2] "SLS Lift Capabilities and Configurations," https://www.nasa.gov/sites/default/files/atoms/files/sls_lift_ capabilities_and_configurations_508_08202018_0.pdf, 2018. Accessed: 31 OCT 2018.

[3] Hemsch, M. J., Hanke, J. L., Walker, E. L., and Houlden, H. P., "Detailed Uncertainty Analysis for Ares I Ascent Aerodynamics Wind Tunnel Database," AIAA Paper 2008-4259, 26 ${ }^{\text {th }}$ AIAA Aerodynamic Measurement Technology and Ground Testing Conference, 23-26 June 2008, Seattle, WA, USA.

[4] Pinier, J. T., Bennett, D. W., Blevins, J. A., Erickson, G. E., Favaregh, N. M., Houlden, H. P., and Tomek, W. G., "Space Launch System Ascent Static Aerodynamic Database Development," AIAA Paper 2014-1254, 52 ${ }^{\text {nd }}$ AIAA Aerospace Sciences Mtg., 13-17 January 2014, National Harbor, MD, USA.

[5] Favaregh, A. L., Houlden, H. P., and Pinier, J. T., "Quantification of the Uncertainties for the Space Launch System Liftoff/Transition and Ascent Databases," AIAA Paper 2016-0795, 54 ${ }^{\text {th }}$ AIAA Aerospace Sciences Mtg., 4-8 January 2016, San Diego, CA, USA.

[6] Hemsch, M. J., "Global Comparison of CFD and Wind-Tunnel-Derived Force and Moment Databases for the Space Launch System,” AIAA Paper 2016-0794, 54 ${ }^{\text {th }}$ AIAA Aerospace Sciences Mtg., 4-8 January 2016, San Diego, CA, USA.

[7] Holmberg, J., "NASA Ames Research Center Wind Tunnel Division, Test Planning Guide for High Speed Wind Tunnels," A027-9391-XB2, Revision 6.

[8] Kmak, F., "Modernization and Activation of the NASA Ames 11- by 11-Foot Transonic Wind Tunnel," AIAA Paper 2000-2680, $21^{\text {st }}$ AIAA Aerodynamic Measurement Technology and Ground Testing Conference, 19-22 June 2002, Denver, CO.

[9] Kmak, F., "Capabilities of the Unitary Plan Wind Tunnel," Oral Presentation, 55 ${ }^{\text {th }}$ AIAA Aerospace Sciences Mtg., 9-13 January 2017, Grapevine, TX, ARC-E-DAA-TN38585.

[10] McMillin, S. N., Shea, P. R., Dalle, D. J., Rogers, S. E., Roozeboom, N. H., Meeroff, J. G., and Lee, H. C., "Comparison of Space Launch System Aerodynamic Surface Pressure Measurements from Experimental Testing and CFD," AIAA Paper, AIAA Aviation 2019 Forum, 17-21 June 2019, Dallas, TX, USA.

[11] Goethert, B. H., Transonic Wind Tunnel Testing, Advisory Group For Aeronautical Research And Development North Atlantic Treaty Organization, 1961. 
[12] Capone, F. J., Bangert, L. S., Asbury, S. C., Mills, C. T. L., and Bare, E. A., "The NASA Langley 16-Foot Transonic Tunnel: Historical Overview, Facility Description, Calibration, Flow Characteristics, and Test Capabilities," NASA TP-3521.

[13] Braslow, A. L., and Knox, E. C., "Simplified Method For Determination Of Critical Height Of Distributed Roughness Particles For Boundary-Layer Transition At Mach Numbers From 0 To 5,” NACA Technical Note 4363, September 1958.

[14] Ulbrich, N., and Volden, T., "Development of a New Software Tool for Balance Calibration Analysis," AIAA Paper 2006-3434, $25^{\text {th }}$ AIAA Aerodynamic Measurement Technology and Ground Testing Conference, 5-8 June 2006, San Francisco, CA.

[15] Ulbrich, N., and Volden, T., "Development of a User Interface for a Regression Analysis Software Tool," AIAA Paper 2010-0932, 48 ${ }^{\text {th }}$ AIAA Aerospace Sciences Mtg., 4-7 January 2010, Orlando, FL.

[16] Meeroff, J. G., Lee, H. C., Dalle, D. J., Rogers, S. E., Roozeboom, N. H., and Baerny, J. K., "Comparison of SLS Sectional Loads from Pressure Sensitive Paint and CFD,” AIAA Paper 2019-2127, AIAA SciTech 2019 Forum, 7-11 January 2019, San Diego, CA, USA.

[17] "MathWorks MATLAB Documentation: scateredInterpolant," https://www.mathworks.com/help/matlab/ref/ scatteredinterpolant.html, 2018. Accessed:19 NOV 2018.

[18] Houlden, H. P., Favaregh, A. L., and Hemsch, M. J., "Uncertainty Quantification and Modeling for Ares I A106 Ascent Aerodynamics Database,” NASA/TM-2013-218045, September 2013.

[19] Houlden, H. P., Favaregh, A. L., and Hemsch, M. J., "Quantification of the Uncertainties for the Ares I A106 Ascent Aerodynamic Database," AIAA Paper 2010-4926, $27^{\text {th }}$ AIAA Aerodynamic Measurement Technology and Ground Testing Conference, 29 June - 1 July 2010, Chicago, IL, USA.

[20] Wheeler, D. J., Advanced Topics in Statistical Process Control, $2^{\text {nd }}$ ed., SPC Press, Knoxville, TN, 2004. 